\title{
Flexible recovery of uniqueness and immutability (Extended Version)
}

\author{
Paola Giannini ${ }^{\mathrm{b}, *}$, Marco Servetto ${ }^{\mathrm{a}}$, Elena Zucca ${ }^{\mathrm{c}}$, James Cone $^{\mathrm{a}}$ \\ ${ }^{a}$ School of Engineering and Computer Science, Victoria University of Wellington, Gate 6, \\ Kelburn Parade Wellington, New Zealand \\ ${ }^{b}$ Computer Science Institute, DiSIT, Università del Piemonte Orientale, viale Teresa \\ Michel 11, Alessandria, Italy \\ ${ }^{c}$ DIBRIS, Università di Genova, Via Dodecaneso 35, Genova, Italy
}

\begin{abstract}
We present an imperative object calculus where types are annotated with qualifiers for aliasing and mutation control. There are two key novelties with respect to similar proposals. First, the type system is very expressive. Notably, it adopts the recovery approach, that is, using the type context to justify strengthening types, greatly improving its power by permitting to recover uniqueness and immutability properties even in presence of other references. This is achieved by rules which restrict the use of such other references in the portion of code which is recovered. Second, execution is modeled by a non standard operational model, where properties of qualifiers can be directly expressed on source terms, rather than as invariants on an auxiliary structure which mimics physical memory. Formally, this is achieved by the block construct, introducing local variable declarations, which, when evaluated, play the role of store.
\end{abstract}

Keywords: Type systems; Imperative calculi; Immutability; Aliasing

\section{Introduction}

In languages with state and mutations, keeping control of aliasing relations is a key issue for correctness. This is exacerbated by concurrency mechanisms, since side-effects in one thread can affect the behaviour of another thread, hence unpredicted aliasing can induce unplanned/unsafe communication.

For these reasons, the last few decades have seen considerable interest in type systems for controlling sharing and interference, to make programs easier to maintain and understand, notably using type qualifiers to restrict the usage of references $30,15,21,11$.

\footnotetext{
*Principal corresponding author

Email addresses: giannini@di.unipmn.it (Paola Giannini), marco.servetto@ecs.vuw.ac.nz (Marco Servetto), elena.zucca@unige.it (Elena Zucca), james.cone@ecs.vuw.ac.nz (James Cone)

This original research has the financial support of the Università del Piemonte Orientale.
} 
In particular, it is very useful for a programmer to be able to rely on the capsule and immutability properties of a reference $x$. To informally explain their meaning, let us consider the store as a graph, where nodes contain records of fields, which may be references to other nodes, and let $x$ be a reference ${ }^{2}$ to a node in the graph. Depending on the qualifier of $x$, restrictions are imposed and assumptions can be made on the whole subgraph reachable from $x$, as detailed below.

If $x$ is capsule, then the subgraph reachable from $x$ is an isolated portion of store, that is, all its (non immutable) nodes can be reached only through this reference. This allows programmers (and static analysis) to identify state that can be safely handled by a thread. In this paper we will use the name capsule for this property, to avoid confusion with many variants in literature [10, 1, 27, 17, 12, 15.

If $x$ is imm (immutable), then the subgraph reachable from $x$ is an immutable portion of store. That is, we impose the restriction that fields of the node cannot be modified ${ }^{3}$ ( $x . f=e$ is not legal), and we can assume that the subgraph reachable from $x$ will not be modified through any other reference. As a consequence, an immutable reference can be safely shared in a multithreaded environment.

Note that the above properties are deep/full, that is, related to the whole object graph. For this reason, unlike early discussions of read-only [5] and the Rust languag $\notin^{4}$ we do not offer any kind of back door to support internal mutability, since this would destroy the immutability assumption on the whole reachable object graph.

In addition to capsule and imm, we will consider three other type qualifiers.

- If $x$ is mut (mutable), then no restrictions are imposed and no assumptions can be made on $x$.

- If $x$ is lent [28, 13, also called borrowed in literature [4, 21, then the subgraph reachable from $x$ can be manipulated, but not shared, by a client. More precisely, the evaluation of an expression which uses a lent reference $x$ can neither connect $x$ to any other external reference, nor to the result of the expression, unless this expression is, in turn, lent.

- Finally, if $x$ is read (readable), then neither modification nor sharing are permitted. That is, we impose both the read-only and the lent (borrowed) restriction; however, note that there is no immutability guarantee.

The last two qualifiers ensure intermediate properties used to derive the capsule and immutable properties.

Whereas (variants of) such qualifiers have appeared in previous literature (see Section 7 for a detailed discussion on related work), there are two key novelties in our approach.

\footnotetext{
fied.

${ }^{2}$ Equivalently, a variable naming a reference: in our formalism the two notions are identi-

${ }^{3}$ This corresponds to the read-only notion in literature.

${ }^{4}$ rust-lang.org
} 
Firstly and more importantly, the type system is very expressive, as will be illustrated by many examples in Section 4 . Indeed, it adopts the recovery technique [15, 11, that is, references (more in general, expressions) originally typed as mutable or readable can be recovered to be capsule or immutable, respectively, provided that no other mutable/readable references are used. However, expressivity of recovery is greatly enhanced, that is, the type system permits recovery of capsule and imm properties in much more situations. Indeed, other mutable/readable references are not hidden once and for all. Instead, they can be used in a controlled way, so that it can be ensured that they will be not referenced from the result of the expression for which we want to recover the capsule or immutability property. Notably, they can be possibly used in some subexpression, if certain conditions are satisfied, thanks to two rules for swapping and unrestricting which are the key of the type system's expressive power.

Secondly, we adopt an innovative execution model [25, 7, 28, for imperative languages which, differently from traditional ones, is a reduction relation on language terms. That is, we do not add an auxiliary structure which mimics physical memory, but such structure is encoded in the language itself. Whereas this makes no difference from a programmer's point of view, it is important on the foundational side, since, as will be informally illustrated in Section 2 and formalized in Section 6, it makes possible to express uniqueness and immutability properties in a simple and direct way.

This paper is an improved and largely extended version of [13]. The novel contributions include reduction rules, more examples and proofs of results.

The rest of the paper is organized as follows: we provide syntax and an informal introduction in Section 2, type system in Section 3, programming examples in Section 4, reduction rules in Section 5, results in Section 6, related work in Section 7, summary of paper contribution and outline of further work in Section 8 . The appendix contains some of the proofs omitted from Section 6 .

\section{Informal presentation of the calculus}

Syntax and types are given in Figure1. We assume sets of variables $x, y, z$, class names $C$, field names $f$, and method names $m$. We adopt the convention that a metavariable which ends by $s$ is implicitly defined as a (possibly empty) sequence, for example $d s$ is defined by $d s::=\epsilon \mid d d s$, where $\epsilon$ denotes the empty sequence.

The syntax mostly follows Java and Featherweight Java (FJ) [18. A class declaration consists of a class name, a sequence of field declarations and a sequence of method declarations. A field declaration consists of a field type and a field name. A method declaration consists, as in FJ, of a return type, a method name, a list of parameter names with their types, and a body which is an expression. However, there is an additional component: the type qualifier for this, which is written as additional (first) element of the parameter list. As in FJ, we assume for each class a canonical constructor whose parameter list exactly corresponds to the class fields, and we assume no multiple declarations of classes in a class table, fields and methods in a class declaration. 


\begin{tabular}{|llll|}
\hline$c d$ & $::=\operatorname{class} C\{f d s m d s\}$ & class declaration \\
$f d$ & $::=\operatorname{imm} C f ; \mid$ mut $C f ; \mid$ int $f ;$ & field declaration \\
$m d$ & $::=T m\left(\mu, T_{1} x_{1}, \ldots, T_{n} x_{n}\right)\{e\}$ & method declaration \\
$e$ & $::=x|e \cdot f| e \cdot m(e s)|e \cdot f=e|$ new $C(e s) \mid\{d s e\}$ & expression \\
$d$ & $::=T x=e ;$ & variable declaration \\
& & \\
$T$ & $::=\mu C \mid$ int & type \\
$\mu$ & $::=\operatorname{mut} \mid$ imm $\mid$ capsule $\mid$ lent $\mid$ read & (type) qualifier \\
& & & \\
$d v$ & $::=T x=r v ;$ & evaluated declaration \\
$r v$ & $::=\operatorname{new} C(x s)|\{d v s x\}|\{d v s$ new $C(x s)\}$ & right-value \\
\hline
\end{tabular}

Figure 1: Syntax and types

For expressions, in addition to the standard constructs of imperative objectoriented languages, we have blocks, which are sequences of variable declarations, followed by a body which is an expression. Variable declarations consist of a type, a variable and an initialization expression. Types are class names decorated by a (type) qualifier. We also include int as an example of primitive type, but we do not formally model related operators used in the examples, such as integer constants and sum. We assume no multiple declarations for variables in a block, that is, $d s$ can be seen as a map from variables into declarations, and we use the notations $\operatorname{dom}(d s)$ and $d s(x)$.

Blocks are a fundamental construct of our calculus, since sequences of local variable declarations, when evaluated, are used to directly represent store in the language itself. A declaration is evaluated if its initialization expression is a right-value, that is, either an object state (a constructor invocation where arguments are references) or a block in which declarations are evaluated and the body is a reference or an object state.

For instanc 5 , assuming that class $B$ has a mut field of type $B$, in the block:

mut $B \mathrm{x}=$ new $\mathrm{B}(\mathrm{y})$; mut $\mathrm{B} y=$ new $\mathrm{B}(\mathrm{x}) ; \mathrm{x}$

the two declarations can be seen as a store where $\mathrm{x}$ denotes an object of class B whose field is y, and conversely, as shown in Figure 2(a). The whole block denotes a store with an entry point (graphically represented by a thick arrow). In our graphical representation circles denote mutable references ( $\mathrm{x}$ and $\mathrm{y}$ in Figure 2), diamonds denote immutable references ( $z$ and $w$ in Figure 2(b)) and squares denote capsule references ( $\mathbf{z}$ in Figure 4).

Stores are hierarchical, rather than flat as it usually happens in models of imperative languages. For instance, assuming that class $\mathrm{C}$ has two mut $\mathrm{D}$ and one imm D fields, and class D has an integer field, the following is a store:

imm $D \quad z=$ new $D(0)$;

imm $C$ w $=\{$ mut $D x=$ new $D(1)$; mut $D y=$ new $D(2)$; new $C(x, y, z)\}$

\footnotetext{
${ }^{5}$ In the examples, we omit for readability the brackets of the outermost block.
} 


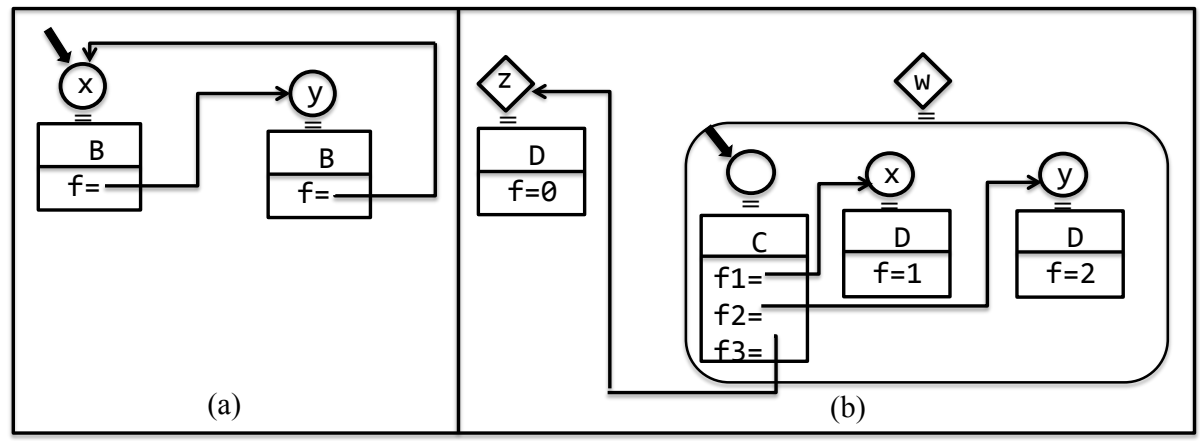

Figure 2: Graphical representation of the store

Here, the right-value associated to w is a block introducing local declarations, that is, in turn a store with an entry point, as shown in Figure 2(b). The advantage of this hierarchical shape is that it models in a simple and natural way constraints about aliasing among objects, notably:

- the fact that an object is not referenced from outside some enclosing object is directly modeled by the block construct: for instance, the objects denoted by $\mathrm{x}$ and $\mathrm{y}$ can only be reached through $\mathrm{w}$

- conversely, the fact that an object does not refer to the outside is modeled by the fact that the corresponding block is closed, that is, has no free variables ${ }^{6}$ for instance, the object denoted by w is not closed, since it refers to the external object $z$.

In the graphical representation the reference corresponding to new $\mathrm{C}(\mathrm{x}, \mathrm{y}, \mathrm{z})$ is anonymous. Note also that, in this example, mutable variables in the local store of $\mathrm{w}$ are not visible from the outside. This models in a natural way the fact that the portion of store denoted by w is indeed immutable, as will be detailed in the sequel.

We illustrate now the meaning of the qualifiers mut, imm, and capsule. A mutable variable refers to a portion of store that can be modified during execution. For instance, the block

mut $B x=$ new $B(y)$; mut $B y=$ new $B(x) ; x \cdot f=x$

reduces to

mut $B \quad x=$ new $B(x)$; mut $B y=$ new $B(x) ; x$

We give a graphical representation of this reduction in Figure 3 where we highlight in grey expressions which are not reduced yet. So in (a) the body of the

\footnotetext{
${ }^{6}$ In other words, our calculus smoothly integrates memory representation with shadowing and $\alpha$-conversion.
} 


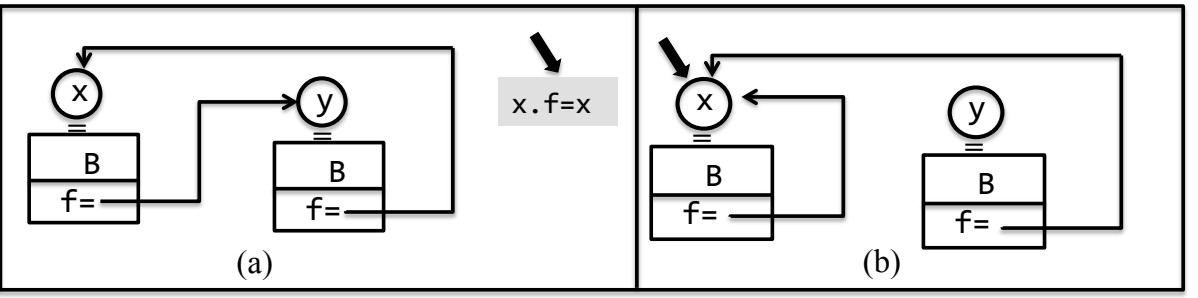

Figure 3: Example of reduction (1)

block is the expression $x . f=x$, whose evaluation modifies the field $f$ of $x$, and returns $\mathrm{x}$. The result of the reduction is shown in (b).

Variables declared immutable, instead, refer to a portion of store which cannot be modified. Immutability is deep/full, that is, all the nodes in the reachable object graph of an immutable reference are immutable themselves. Therefore, in the enclosing scope of the declaration

imm $C$ w $=\{$ mut $D x=$ new $D(1)$; mut $D y=$ new $D(2)$; new $C(x, y, z)$ \}

the variable $\mathbf{z}$ must be declared $\mathbf{i m m}$, and we cannot have an assignment to a field of $w$.

A variable declared capsule refers to an isolated portion of store, where local objects can freely reference each other but for which the variable is the only external reference. For instance in:

capsule $B z=\{$ mut $B \quad x=$ new $B(y) ;$ mut $B y=$ new $B(x) ; x\}$

the internal objects denoted by $\mathrm{x}$ and $\mathrm{y}$ can be only be accessed through $\mathrm{z}$. A capsule variable can be used once and for all to "move" an isolated portion of store to another node in the store. To get more flexibility, external immutable references are freely allowed. For instance, in the example above of the declaration of w, the inizialization expression has a capsule type. In our type system, capsule types are subtypes of both mutable and immutable types. Hence, capsule expressions can initialize both mutable and immutable references. To preserve the capsule property, we need a linearity constraint: that is, a syntactic constraint that in well-formed expressions capsule references can occur at most once in their scope (see more comments at page 25).

Consider the term

mut $\mathrm{D}$ y=new $\mathrm{D}(0)$;

capsule $C z=\{$ mut $D \quad x=$ new $D(y \cdot f=y \cdot f+1) ;$ new $C(x, x)\}$

In Figure 4(a) we have a graphical representation of this term. The evaluation of the expression on the right-hand side of $x$ starts by evaluating $y . f+1$, which triggers the evaluation of y.f. The result is shown in (b), then the sum $0+1$ is evaluated, returning 1 , as shown in (c). The evaluation of the field assignment $y \cdot f=1$ updates the field $f$ of $y$ to 1 , and 1 is returned. Since new $D(1)$ is a value, the whole term is fully evaluated, and it is shown in (d). 


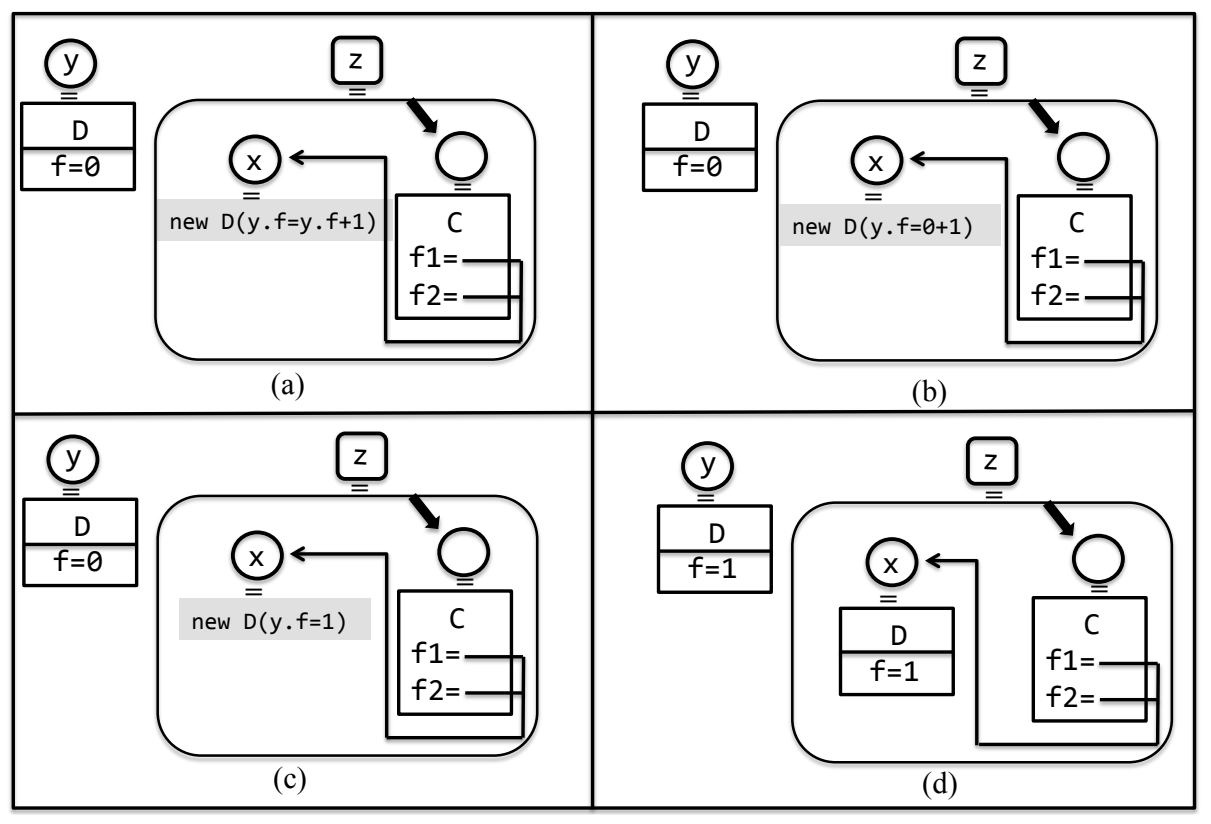

Figure 4: Example of reduction (2)

To be able to typecheck more expressions as capsule or imm, we introduce the lent and read qualifiers. References with such qualifiers can be used in a restricted way. That is, no aliasing can be introduced between a lent reference and another reference, and a lent reference cannot be part of the result of the expression where it is used, unless this expression is, in turn, lent. A read reference is lent and, moreover, cannot be modified.

The relation $\mu \leq \mu^{\prime}$ intuitively means that qualifier $\mu^{\prime}$ imposes more restrictions than $\mu$, hence a $\mu$ reference can be safely used where a $\mu^{\prime}$ is required. Notably, lent and imm references can be used where a read is required, a mutable reference where a lent is required, and a capsule reference can be used everywhere.

Altogether, the subtyping relation is the reflexive and transitive relation on types induced by

$$
\begin{aligned}
& \text { int } \leq \text { int } \\
& \mu C \leq \mu^{\prime} C \text { if } \mu \leq \mu^{\prime} \\
& \text { capsule } \leq \text { mut } \leq \text { lent } \leq \text { read } \\
& \text { capsule } \leq \text { imm } \leq \text { read }
\end{aligned}
$$

Note that capsules can be used as mutable or immutable, with the constraint that capsule references can be used only once. So, a capsule reference can be seen as a reference whose destiny has not been decided yet.

In some cases it is possible to move the type of an expression against the 


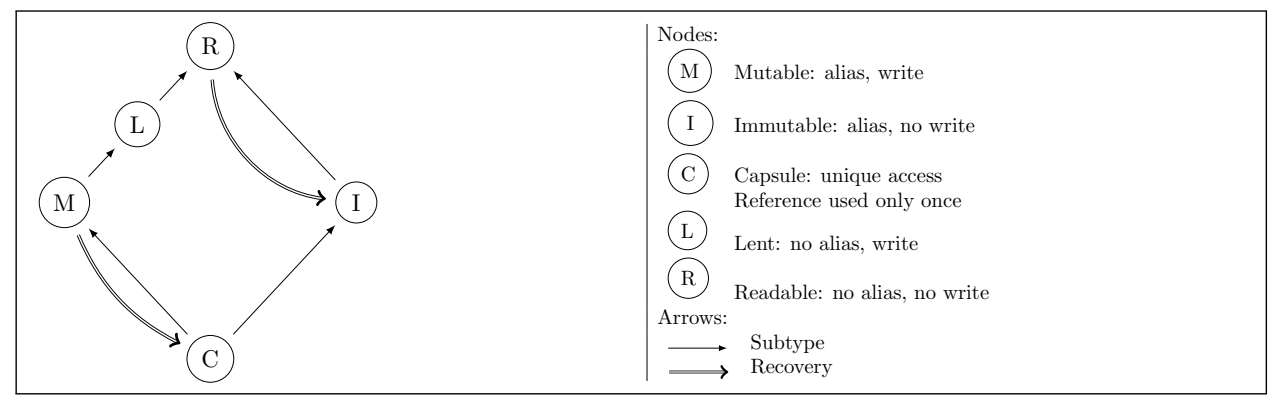

Figure 5: Type qualifiers and their relationships

subtype hierarchy. Notably, we can recover the capsule property for a mut expression, and the imm property for a read expression, provided that some of the free variables in the expression are used in a controlled way. Recovery will be described in the following section.

The situation is graphically depicted in Figure 5

\section{Type system}

Type contexts are defined by:

$$
\begin{aligned}
& \Delta::=\Gamma ; x s ; y s \quad \text { type context } \\
& \Gamma \quad::=x_{1}: T_{1}, \ldots, x_{n}: T_{n} \text { type assignment }
\end{aligned}
$$

In a type context $\Delta=\Gamma ; x s s ; y s, \Gamma$ is a usual assignment of types to variables. We write $\operatorname{dom}(\Gamma)$ for the set of variables in $\Gamma$, and $\Gamma\left[\Gamma^{\prime}\right]$ for the concatenation of $\Gamma$ and $\Gamma^{\prime}$ where, for the variables occurring in both domains, $\Gamma^{\prime}$ takes precedence. We will also use $\operatorname{dom}^{\text {mut }}(\Gamma)$ for the set of mutable variables in $\Gamma$, and other analogous notations.

According to our convention, xss is a sequence $x s_{1} \ldots x s_{n}$ of sequences of variables, and $y s$ is a sequence of variables. All such sequences are assumed to be sets (that is, order and repetitions are immaterial). The sets $x s_{1} \ldots x s_{n}$ are assumed to be pairwise disjoint, and are called lent groups, whereas the mut variables in $\Gamma$ which do not belong to any lent group form the mutable group. Finally, variables in ys are called restricted variables.

Lent groups and restricted variables are the key novelty of our type system. The property they ensure is that, if $\Gamma$; xss; ys $\vdash e: T$, then the final result of $e$ will be not connected to variables which are in xss or ys. In this way, to ensure that the final result of $e$ is an isolated portion of store, it is enough to typecheck $e$ in a type context where all its free mutable variables are in a lent group or restricted. This is a crucial improvement with respect to previous mechanisms of recovery [15, 11, where this could be only ensured if $e$ had only immutable or isolated (capsule in our teminology) free variables. We have distinct lent groups $x s_{1} \ldots x s_{n}$ since there can be nested recoveries, hence, to ensure that all are safe, we need also the auxiliary property that no aliasing is introduced 
between different groups, as will be illustrated in detail by the last example of Section 4 That is, if the reachable graphs of $x s_{i}$ and $x s_{j}$ were disjoint before the evaluation of the expression, then after the evaluation they should be still disjoint.7

The type system ensures the above properties by imposing that variables in xss and ys are used in controlled way:

- A mut variable in $\Gamma$ which belongs to a lent group $x s_{i}$ can only be used as lent. Notably, write access is not directly allowed since it could introduce aliasing.

- A read, lent or mut variable in $\Gamma$ which is restricted cannot be used at all, that is, is hidden 8

The important point is that such constraints are not imposed once and for all when typechecking an expression. That is, a subexpression can be typechecked with different lent groups and restricted variables, more precisely:

- In a subexpression we can swap one of the lent groups $x s_{i}$ with the mutable group, weakening to lent the type of the subexpression if it was mutable. In this way, write access to variables in $x s_{i}$ is allowed, but this is safe since the result of the subexpression is in turn lent, so no aliasing can be introduced with the result of the main expression.

- In a subexpression we can unrestrict variables in ys, that is, freely use such variables, provided that the type of the subexpression is imm or capsule. Again this guarantees that no aliasing can be introduced with the result of the main expression.

Lent groups and restricted variables are introduced as an effect of applying recovery rules (T-CAPsule) and (т-Iмм), whereas swapping and unrestricting are obtained by applying rules (T-SWAP) and (T-UNRST) rules, as will be explained in detail later.

Contexts $\Delta=\Gamma$; xss; ys are well-formed, written $\vdash \Delta$, if, for $x s s=x s_{1} \ldots x s_{n}$, the following conditions hold:

- no variable is lent in $\Gamma$

- if $x \in x s_{i}$ for some $i \in 1 . . n$, then $x$ is mut in $\Gamma$

- if $y \in y s$, then $y$ is mut or read in $\Gamma$

- if $y \in y s$ and is mut in $\Gamma$, then $y \in x s_{i}$ for some $i \in 1$..n.

\footnotetext{
${ }^{7}$ Note that this is different from regions as in, e.g., [19] [16], which are sets of references assumed to be disjoint.

${ }^{8}$ However, we use the terminology "restricted" since this hiding is non permanent.
} 
We do not consider lent variables in $\Gamma$ since assigning the type lent $C$ to a variable $x$ is encoded by assigning to $x$ the type mut $C$ and having $x$ in a lent group in xss (see the explanation of rule (т-вцоск) in the following). The last condition requires coherency between xss and ys, that is, restricted mutable variable are in a lent group. It is easy to check that well-formedness of contexts is preserved in type derivations. That is, if $\Delta \vdash e: T$ and $\Delta$ is well-formed, then in all sub-derivations the contexts are well-formed. In the following when we write $\Delta \vdash e: T$ we assume that $\Delta$ is well-formed.

In the rules we use information extracted from the class table, which is modelled, as usual, by the following functions:

- fields $(C)$ gives, for each declared class $C$, the sequence of its fields declarations

- method $(C, m)$ gives, for each method $m$ declared in class $C$, the tuple $\left\langle T, \mu, T_{1} x_{1} \ldots T_{n} x_{n}, e\right\rangle$ consisting of its return type, type qualifier for this, parameters, and body.

We assume method bodies to be well-typed w.r.t. the type annotations in the method declaration. More precisely, if

$$
\operatorname{method}(C, m)=\left\langle T, \mu, T_{1} x_{1} \ldots T_{n} x_{n}, e\right\rangle
$$

then

$$
\Gamma ; x s s ; \emptyset \vdash e: T
$$

where xss consists of the singletons of the parameters declared lent, including the implicit parameter this, expressing the requirement that they should not be aliased by the method, and $\Gamma=$ this: $\mu^{\prime} C, x_{1}: T_{1}^{\prime}, \ldots, x_{n}: T_{n}^{\prime}$, where $T_{i}=\operatorname{mut} C_{i}$ if $T_{i}=$ lent $C_{i}, T_{i}^{\prime}=T_{i}$ otherwise, and $\mu^{\prime}=$ mut if $\mu=$ lent, $\mu^{\prime}=\mu$ otherwise.

Typing rules are given in Figure 6. In the typing rules, when we need to make explicit the mutable group, we use the auxiliary judgment $\Gamma$; xss $[x s]$; ys $\vdash$ $e: T$, which stands for the judgment $\Gamma$; xss; ys $\vdash e: T$ with the side condition $x s=\operatorname{dom}^{\text {mut }}(\Gamma) \backslash x s s$, meaning that $x s$ are the mut variables in $\Gamma$ which do not belong to any lent group in xss.

Rules (т-сAPsule) and (т-імm) model recovery, that is, can be used to recover a more specific type for an expression, under the conditions that the use of some free variables in the expression is controlled. There are two kinds of recovery:

- mut $\Rightarrow$ capsule

As shown in rule (T-Capsule), an expression can be typed capsule in $\Gamma$; xss; ys if it can be typed mut by turning in lent the mutable group (xs), which becomes empty. Formally, this group is added to xss.

- read $\Rightarrow$ imm

As shown in rule (T-IMm), an expression can be typed imm in $\Gamma$; xss; ys if it can be typed read by turning in lent the mutable group (xs), as in the recovery above, and, moreover, restricting currently available mutable, lent, and readable variables $\left(\mathrm{dom}^{\geq \operatorname{mut}}(\Gamma)\right)$. 


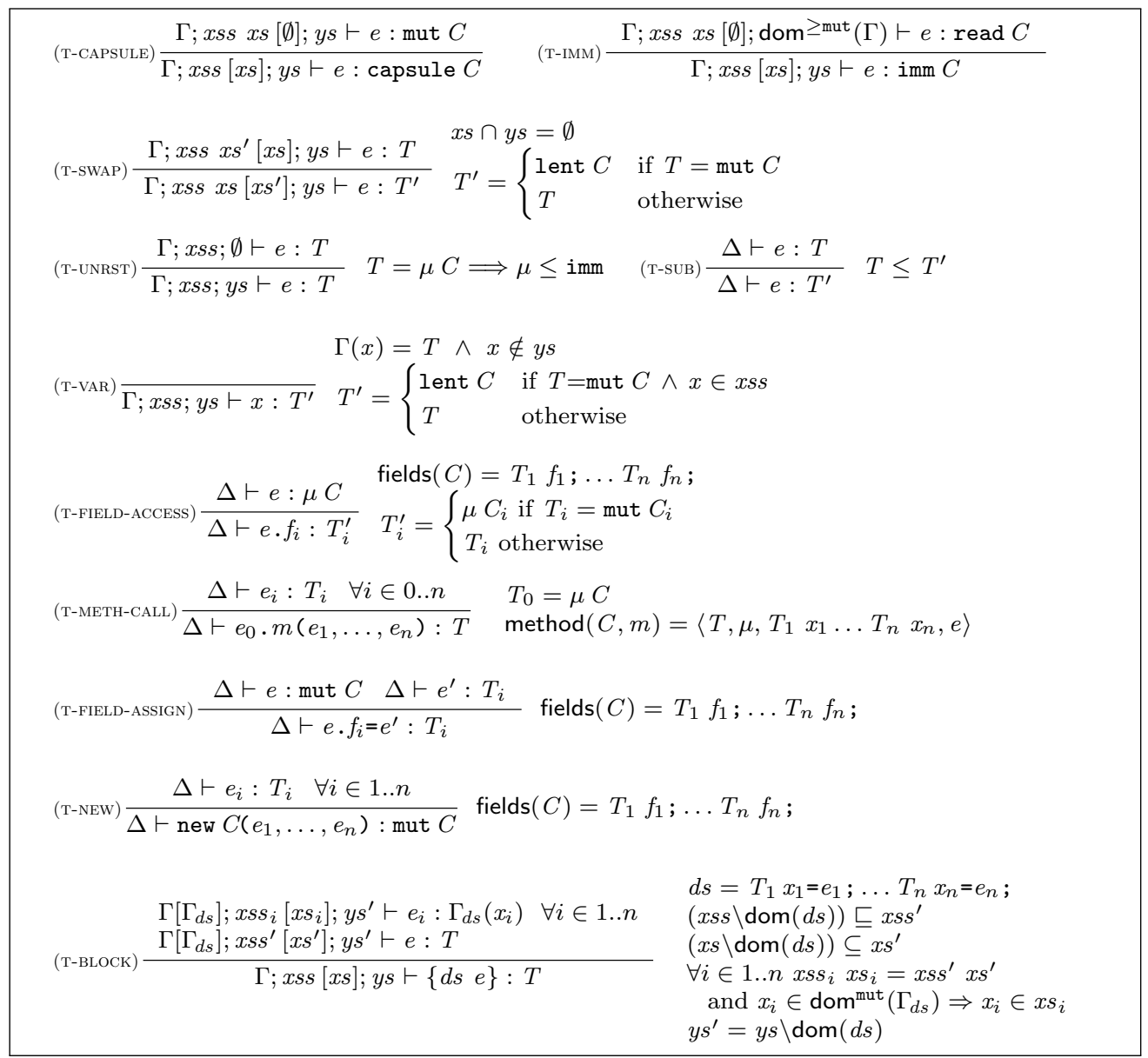

Figure 6: Typing rules

Along with recovery rules which introduce lent groups and restricted variables, we have two corresponding elimination rules. In the detail:

- (T-SWAP)

An expression can be typed in $\Gamma$; xss xs; ys if it can be typed by turning into mutable some lent group (xs), by swapping this group with the current mutable group $\left(x s^{\prime}\right)$. The side condition $x s \cap y s=\emptyset$ prevents to swap a lent group including restricted variables. The type obtained in this way is weakened to lent, if it was mut.

- (T-UNRST)

An expression can be typed in $\Gamma$; xss; ys if it can be typed by unrestricting all restricted variables ys, provided that the type obtained in this way is capsule or imm or a primitive type. 
Note that, without these last two rules, recovery rules would be essentially equivalent to those in prior work [15, 11. Rules (T-SWAP) and (T-UNRST) add power to the type system, and they are also the reason it requires the xss and ys information during typechecking. Prior work [15, 11] can afford to simply locally using weakening and subtyping to make references inaccessible or convert mut references to lent.

Note also that recovery rules, (T-SWAP), and (T-UNRST) are not syntax-directed, analogously to the subsumption rule. In other words, their functionality does not restrict how code is written: they are transparent to the programmer, in the sense that they are applied when needed. The programmer can simply rely on the fact that expressions are capsule or imm, respectively, in situations where this is intuitively expected, as we illustrate by the following examples (other will be provided in next section).

We explain now in detail how recovery, swapping and unrestricting work, then comment the other rules.

Capsule recovery. Let us discuss, first, when an expression can be safely typed capsule. The evaluation of the expression should produce a portion of store which is isolated, apart from external immutable references, formally a rightvalue where all free variables are immutable. Obviously, this is safe for an expression which has itself no free variables, or where all the free variables are imm or capsule, and, indeed, this was the requirement needed for obtaining recovery in previous work [15. However, this requirement is too strong. Consider the following sequence of declarations:

mut $D$ y=new $D(0)$;

capsule $C z=\{$ mut $D x=$ new $D(y . f)$; new $C(x, x)\}$;

The inner block (right-hand side of the declaration of $z$ ) can be typed capsule, even though there is a mutable free variable $y$, since this variable is only used in a field access, hence, intuitively, no aliasing is introduced between $\mathrm{y}$ and the final result of the block. Indeed, the block reduces $\mathrm{g}^{9}$ to

$\{$ mut $\mathrm{D} x=$ new $\mathrm{D}(0)$; new $\mathrm{C}(\mathrm{x}, \mathrm{x})\}$;

which is closed. To allow such typing, the inner block is typed applying rule (T-CAPSULE), since it can be typechecked mut in a type context where variable y is lent. In Figure 7 we show the type derivation for this example, where, for clarity, we always write the mutable group even when it is empty.

Note that in an analogous example where the field of class D has a non primitive type, e.g., String:

mut $D$ y=new D("hello");

capsule $C z=\{$ mut $D x=$ new $D(y . f)$; new $C(x, x)\}$;

\footnotetext{
${ }^{9}$ As formalized in Section 5
} 


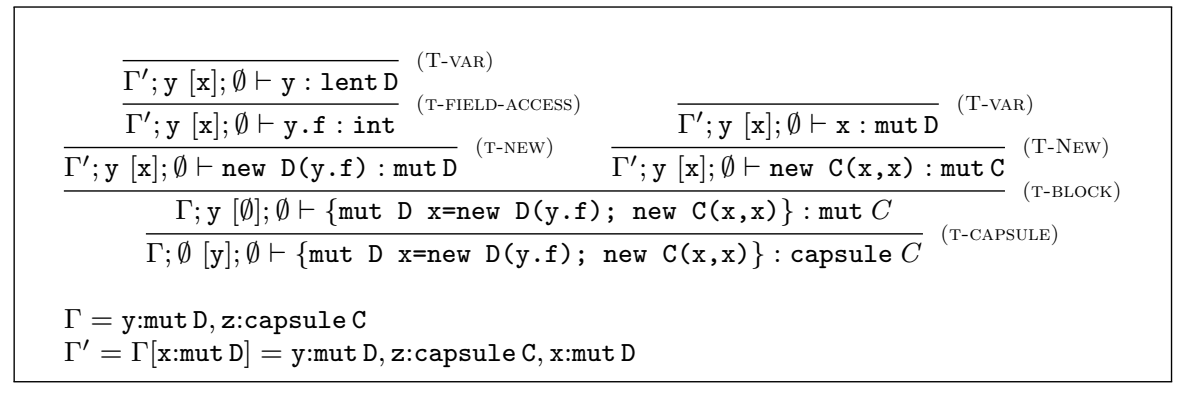

Figure 7: Example of type derivation (1)

the qualifier of the field should be imm, since, otherwise, by introducing a singleton lent group y we would get a lent type for y.f as well, see rule (T-FIELD-ACCESS), and a lent type is not accepted for a constructor argument.

As a counterexample, consider the following sequence of declarations:

mut $D$ y=new $D(0)$;

capsule $C \mathrm{z}=\{$ mut $\mathrm{D} \mathrm{x}=\mathrm{y}$; new $\mathrm{C}(\mathrm{x}, \mathrm{x})\}$;

Here the inner block cannot be typed capsule, since y is internally aliased. Indeed, the block reduces to new $\mathrm{C}(\mathrm{y}, \mathrm{y})$ which contains a free mutable variable. Formally, we cannot apply rule (T-CAPSULE) on the block, since we should typecheck the block with y in a singleton lent group, while the initialization expression of $\mathrm{x}$ should be mutable, see rule (т-вьоск).

Rule (T-FIELD-ASSIGN) requires a mutable type for the receiver. So, how is it possible to modify (the portion of store denoted by) a lent reference? Consider the following simple example:

lent $\mathrm{D} y=$ new $\mathrm{D}(0)$;

$y \cdot f=y \cdot f+1$;

This code should be well-typed, since the assignment does not introduce any alias. To get such typing, we use rule (T-SWAP) to type the expression y. $f=y \cdot f+1$. Indeed, we can swap the singleton lent group y with the empty set.

Moreover, swapping can be applied to achieve recovery. Take the example already considered at page 6

mut $D$ y=new $D(0)$;

capsule $C z=\{$ mut $D \quad x=$ new $D(y \cdot f=y \cdot f+1) ;$ new $C(x, x)\}$

Let $e$ be the inner block (right-hand side of the declaration of $\mathbf{z}$ ). As in the first example, $e$ can be typed capsule if it can be typed mut in a context with type assignment y:mut D, z:capsule $C$ and the lent group y. However, the assignment $y \cdot f=y \cdot f+1$ is not well-typed in this type context, since the variable y has type lent D. However, intuitively, we can see that the assignment does not introduce any alias between $\mathrm{y}$ and the final result of $e$, since it involves only variables which are in the same group (the singleton y), and produces a result which is not mutable. In other words, the result of the evaluation of $e$ is 


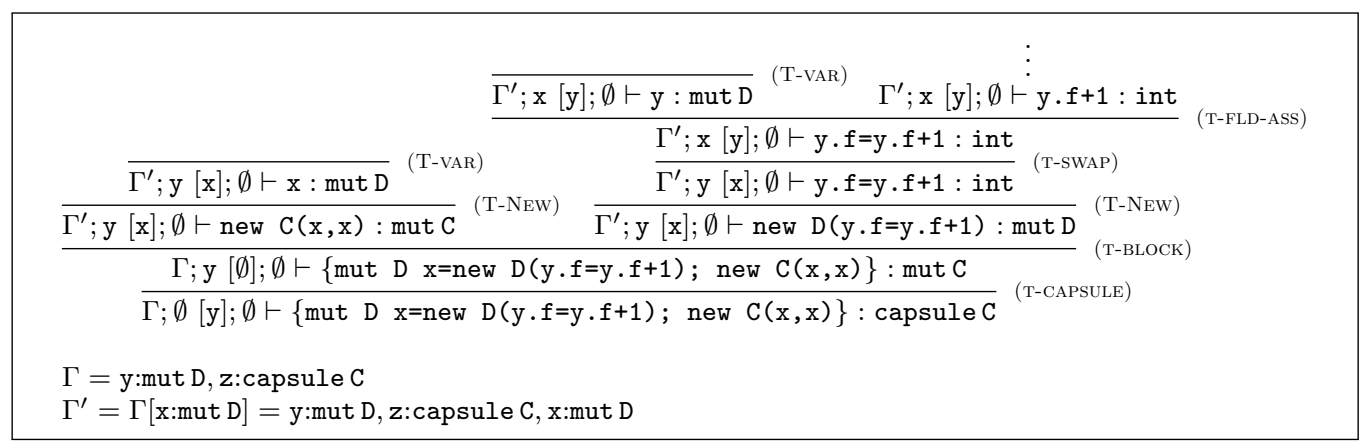

Figure 8: Example of type derivation (2)

a capsule, as it has been shown in Figure 4, so it should be possible to type the expression $e$ as capsule.

To get such typing, we can apply rule (T-SWAP) when deriving the type for the subexpression $y \cdot f=y \cdot f+1$, by swapping the group y with the group $x$. This ensures that the evaluation of the subexpression typed by this rule will not introduce any alias between the variables in the swapped group and the mutable group. The type derivation for the example is given in Figure 8

Note that, when using rule (T-SWAP) to typecheck a subexpression of an expression for which we want the capsule or immutability property, no alias should be introduced between the variables in the group $x s$ and the result of the expression. Indeed, in this case the result of the subexpression could contain references to the variables in group $x s$, which was lent in the original context.To ensure this, the type obtained in this way is weakened to lent, if it was mut. This is shown by the following example:

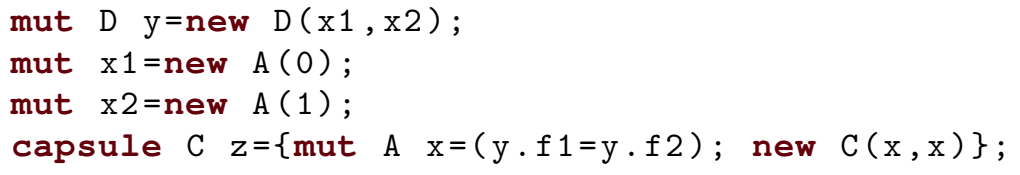

If we apply rule (T-SwAP) when deriving the type for y.f1=y.f2, therefore swapping the group y with $\mathrm{x}$, then we derive type mut $\mathrm{A}$, and rule (T-SwAP) would assign type lent A to the expression. Therefore, the declaration mut A $x=(y \cdot f 1=y \cdot f 2)$ and the whole expression would be ill-typed. Indeed, the expression reduces to

mut $\mathrm{D} y=$ new $\mathrm{D}(\mathrm{x} 2, \mathrm{x} 2)$;

mut $\mathrm{x} 1=$ new $\mathrm{A}(0)$;

mut $\times 2=$ new $A(1)$;

capsule $C$ z=new $C(x 2, x 2)$;

in which the value of $z$ is not a capsule.

Immutability recovery. Note that capsule recovery can only happen for mut expressions. In other words, mut expressions which reduce to a portion of store 
with no external mutable references can be safely used where (either a mutable or) an immutable is required. Indeed, every expression which can be typed capsule can be typed imm by subtyping.

Consider now an expression for which capsule recovery cannot happen, that is, which can be typed lent or read, but cannot be typed mut, hence should not be used where a mut is required. We can directly ${ }^{10}$ recover immutability for such an expression, if we can guarantee that the result of the expression will be not connected to external mutable references. This can be ensured as for the case of capsule recovery, with one difference. For capsule recovery, lent and read references can be freely used, since in any case they will be not connected to the final result of the expression. However, if the expression is in turn lent or read, its result could be connected to lent or read references, hence this should be explicitly prevented by the type system. This is achieved by restricting such references, that is, allowing their use only to typecheck subexpressions of imm capsule or primitive type.

Consider the following variant of the first example of capsule recovery:

mut $\mathrm{D}$ y=new $\mathrm{D}(0)$;

imm $C z=\{$ lent $D x=$ new $D(y . f)$; new $C(x, x)\}$;

As in the original version, the inner block (right-hand side of the declaration of $z$ ) uses the mutable free variable $y$ only in a field access, and indeed reduces to the block $\{$ lent $\mathrm{D} x=$ new $\mathrm{D}(0)$; new $\mathrm{C}(\mathrm{x}, \mathrm{x})$ \} which is closed. However, this block cannot be typed capsule, since it cannot be safely assigned to a mutable reference. On the other hand, the block can be safely typed imm, since, intuitively, it reduces to a portion of store which cannot be modified through any other reference. Formally, the inner block can be typed imm by rule (тгмм), since it can be typechecked lent in a type context where variable y is in singleton lent group and, moreover, restricted, that is, can be only used to typecheck subexpressions which are imm, capsule, or of a primitive type. The type derivation for the example is given in Figure 9. Note that, instead of putting $\mathrm{x}$ is a sigleton group we could have put $\mathrm{x}$ and $\mathrm{y}$ in the same group in the typing of new $C(x, x)$ and new $D(y . f)$. That is, replacing $\{x\}\{y\}$ with $\{\mathrm{x}, \mathrm{y}\}$ the derivation would still be correct. (Clearly the rule (T-Swap) would swap $\{\mathrm{x}, \mathrm{y}\}$ with the empty mutable group.)

Restricting $y$ prevents typechecking examples like the following:

mut $\mathrm{D}$ y=new $\mathrm{D}(0)$;

imm $C z=\{$ lent $D x=y ;$ new $C(x, x)\}$;

The significance of the immutability recovery is more clearly shown by considering method calls, as will be illustrated in Section 4 .

Blocks. A block $\{d s e\}$, where $d s=T_{1} x_{1}=e_{1} ; \ldots T_{n} x_{n}=e_{n}$; , is well-typed if the right-hand sides of declarations and the body are well-typed, as detailed below.

\footnotetext{
${ }^{10}$ That is, not by subtyping.
} 


$$
\begin{aligned}
& \overline{\Gamma^{\prime} ;\{\mathrm{y}\}\{\mathrm{x}\}[\emptyset] ; \emptyset \vdash \mathrm{y}: \text { lent } \mathrm{D}} \\
& \overline{\Gamma^{\prime} ;\{\mathrm{y}\}\{\mathrm{x}\}[\emptyset] ; \emptyset \vdash \mathrm{y} \cdot \mathrm{f} \cdot \text { int }} \text { (T-FIELD-ACCESS) } \\
& \overline{\Gamma^{\prime} ;\{\mathrm{y}\}\{\mathrm{x}\}[\emptyset] ; \mathrm{y} \vdash \mathrm{y} . \mathrm{f}: \text { int }} \text { (T-UNRST) } \\
& \overline{\Gamma^{\prime} ;\{\mathrm{y}\}\{\mathrm{x}\}[\emptyset] ; \mathrm{y} \vdash \text { new } \mathrm{D}(\mathrm{y} . \mathrm{f}): \operatorname{mut} \mathrm{D}}\left(\text { T-New) } \quad \frac{\Gamma^{\prime} ;\{\mathrm{y}\}\{\mathrm{x}\}[\emptyset] ; \mathrm{y} \vdash \text { new } \mathrm{C}(\mathrm{x}, \mathrm{x}): \text { lent }}{\Gamma^{\prime}}\right. \\
& \frac{\Gamma ; \mathrm{y}[\emptyset] ; \mathrm{y} \vdash\{\text { lent } \mathrm{D} x=\text { new } \mathrm{D}(\mathrm{y} . \mathrm{f}) ; \text { new } \mathrm{C}(\mathrm{x}, \mathrm{x})\}: \operatorname{lent} \mathrm{C}}{\Gamma ; \emptyset[\mathrm{y}] ; \emptyset \vdash\{\text { lent } \mathrm{D} \text { x=new } \mathrm{D}(\mathrm{y} . \mathrm{f}) ; \text { new } \mathrm{C}(\mathrm{x}, \mathrm{x})\}: \operatorname{imm} \mathrm{C}} \text { (T-IMM) } \\
& \Gamma=\mathrm{y}: \text { mut } \mathrm{D}, \mathrm{z}: \text { imm C } \\
& \Gamma^{\prime}=\Gamma[\mathrm{x}: \text { mut } \mathrm{D}]=\mathrm{y}: \text { mut } \mathrm{D}, \mathrm{z} \text { :imm C, x:mut D }
\end{aligned}
$$

Figure 9: Example of type derivation (3)

- All the expressions are typechecked w.r.t. the type assignment $\Gamma\left[\Gamma_{d s}\right]$ where $\Gamma_{d s}$ is the same of $x_{1}: T_{1}, \ldots, x_{n}: T_{n}$, apart that local variables declared lent have type mut (indeed the fact that they are lent is encoded by including them in lent groups, see next point).

- The body is typechecked w.r.t. lent groups $x s s^{\prime}$ and mutable group $x s^{\prime}$ which extend those of the enclosing scope, modulo hiding (second and third side conditions). More precisely: variables which are mut in $\Gamma_{d s}$ can be possibly added to a lent group of the enclosing scope, or can form a new lent group, or can be added to the mutable group $x s$ of the enclosing scope.

- The right-hand side of each declaration $e_{i}$ is typechecked w.r.t. to lent groups $x s s_{i}$ and mutable group $x s_{i}$ obtained from those of the body by swapping $x s^{\prime}$ with the group which contains $x_{i}$, if $x_{i}$ is mut in $\Gamma_{d s}$ (fourth side condition). Recall that sequences of groups are considered as sets, hence the notation $x s s_{i} x s_{i}=x s s^{\prime} x s^{\prime}$ means that the two sides are the same set of groups. This swapping models the fact that the initialization expression of a variable $x_{i}$ in a lent group is typechecked considering as mutable group that containing $x_{i}$. For variables in the mutable group, or declared with other modifiers, no swapping is needed.

- All the expressions are typechecked w.r.t. restricted variables which are exactly those of the enclosing scope (modulo hiding).

We use the following auxiliary notations.

- The type assignment extracted from a sequence of declarations $d s$, denoted $\Gamma_{d s}$, is defined by: $\Gamma_{T_{1} x_{1}=e_{1} ; \ldots T_{n} x_{n}=e_{n} ;}=x_{1}: T_{1}^{\prime}, \ldots, x_{n}: T_{n}^{\prime}$ where $T_{i}^{\prime}=$ mut $C$ if $T_{i}=$ lent $C, T_{i}^{\prime}=T_{i}$ otherwise.

- $\left(x s_{1} \ldots x s_{n}\right) \backslash x s=\left(x s_{1} \backslash x s\right) \ldots\left(x s_{n} \backslash x s\right)$

- $\operatorname{dom}\left(x s_{1} \ldots x s_{n}\right)=\left\{x \mid x \in x s_{i}\right.$ for some $\left.i\right\}$ 
- $x s_{1} \ldots x s_{n} \sqsubseteq y s_{1} \ldots y s_{m}$ if $\operatorname{dom}\left(x s_{1} \ldots x s_{n}\right) \subseteq \operatorname{dom}\left(y s_{1} \ldots y s_{m}\right)$, and for all $x, y \in \operatorname{dom}\left(x s_{1} \ldots x s_{n}\right), x, y \in x s_{i}$ if and only if $x, y \in y s_{j}$.

Note that local variables declared lent can be arbitrarily assigned to lent groups, to improve expressivity. For instance, it can be necessary to assign a lent local variable to the same lent group as some variables of the enclosing scope. This is shown by the following example, where the local variable $z 1$ is used to modify the external reference $\mathrm{x}$, rather than to construct the block result.

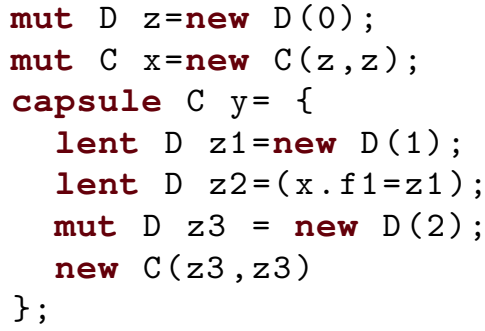

Since we need to recover the capsule property for the block on the right-hand side of the declaration of y, applying rule (T-CAPSULE) to the block, the context of the typing of such block must be

$\mathrm{z}$ : mut $\mathrm{D}, \mathrm{x}$ : mut $\mathrm{C}, \mathrm{y}$ : capsule $\mathrm{C} ;\{\mathbf{z}, \mathrm{x}\} ; \emptyset$

that is, the variables $\mathbf{z}$ and $\mathbf{x}$ must be in the same lent group. However, assuming that field $\mathrm{f} 1$ is mutable, to apply rule (T-Field-Assign) to the expression $\mathrm{x} . \mathrm{f} 1=\mathrm{z} 1$, both $\mathrm{x}$ and $\mathrm{z} 1$ have to be mutable. Therefore, we have to apply rule (T-SwAP), and it must be the case that $\mathrm{x}$ and $\mathrm{z} 1$ are in the same lent group. This is possible, with rule (т-вцоск), by adding $z 1$ to the group $\{z, x\}$ in typing the right-hand sides of the declarations and the body.

Note that the following variant of the example, where the result of the block is connected to $z 1$ instead,

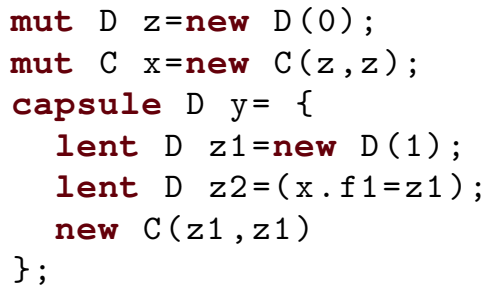

is ill-typed. Indeed, as before, $\mathrm{z} 1$ must be in the same group as $\mathrm{x}$ in order to recover the capsule property of the block, but in this case $z 1$ would be lent, hence the whole block.

Other typing rules. Other rules are mostly standard, except that they model the expected behaviour of type qualifiers.

In rule (T-VAR), a variable is weakened to lent if it belongs to some group in $x s s$, and cannot be used at all if it belongs to $y s$. 
In rule (T-FIELD-ACCESS), in case the field is mut, the type qualifier of the receiver is propagated to the field. For instance, mutable fields referred through a lent reference are lent as well. If the field is immutable (or of a primitive type), instead, then the expression has the field type, regardless of the receiver type.

In rule (T-FIELD-ASSIGN), the receiver should be mutable, and the right-hand side must have the field type. Note that this implies the right-hand side to be either mut or imm (or of a primitive type). Hence, neither the left-hand nor the right-hand sides can be lent or read. This prevents the introduction of aliases for such references. However, recall that for lent references the constraint can be escaped by using, before this rule, rule (T-SWAP), at the price of weakening to lent the type of the expression.

In rule (T-NEW), expressions assigned to fields should be either mut or imm (or of a primitive type). Again, for lent references the constraint can be escaped by swapping, getting a lent expression. Note that an object is created with no restrictions, that is, as mut.

Finally, note that primitive types are used in the standard way. For instance, in the premise of rule (T-NEW) the types of constructor arguments could be primitive types, whereas in rule (T-метн-СALL) the type of receiver could not.

\section{Programming examples}

In this section we illustrate the expressivity of the type system by more significant examples. For sake of readability, we use additional constructs, such as operators of primitive types, static methods, private fields, and while loops. Moreover, we generally omit the brackets of the outermost block, and abbreviate $\left\{T x=e ; e^{\prime}\right\}$ by $e ; e^{\prime}$ when $x \notin \mathrm{FV}\left(e^{\prime}\right)$, with $\mathrm{FV}(e)$ the set of the free variables of $e$.

Capsules and swapping. The following example illustrates how a programmer can declare lent references to achieve recovery. The class CustomerReader below models reading information about customers out of a text file formatted as shown in the example:

\section{$\mathrm{Bob}$}

150021300

Mark

$428899 \quad 100$

In even lines we have customer names, in odd lines we have a shop history: a sequence of product codes.

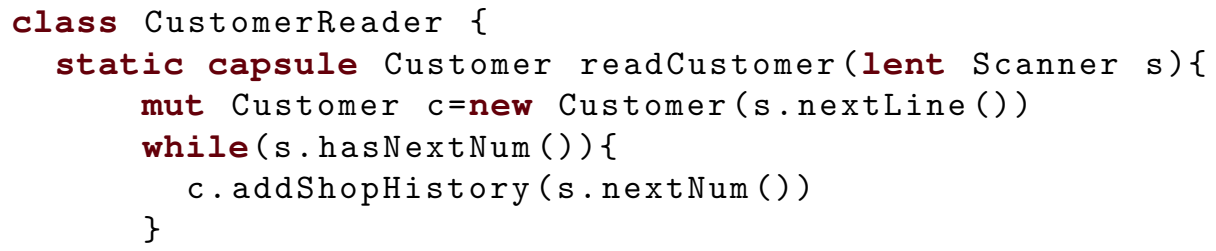




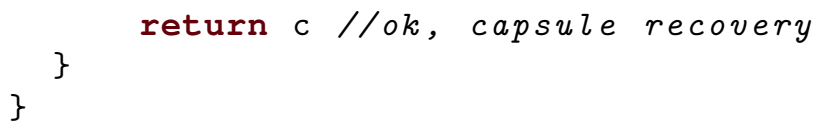

The method CustomerReader.readCustomer takes a lent Scanner, assumed to be a class similar to the one in Java, for reading a file and extracting different kinds of data. A Customer object is created reading its name from the file, and then its shop history is added. Since the scanner is declared lent, and there are no other parameters, by recovery the result can be declared capsule. That is, we can statically ensure that the data of the scanner are not mixed with the result. Previous work offers cumbersome solutions requiring the programmer to manually handle multiple initialization phases like "raw" and "cooked" 30.

The following method update illustrates how we can "open" capsules, modify their values and then recover the original capsule guarantee. The method takes an old customer (as capsule) and a lent Scanner as before.

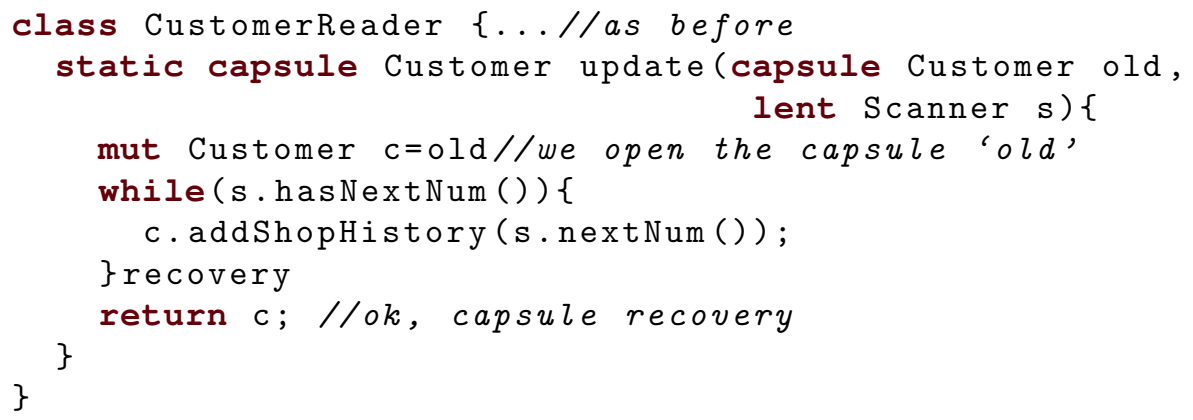

Every method with no mutable parameters can use the pattern illustrated above: one (or many) capsule parameters are opened (that is, assigned to mutable local variables) and, in the end, the result is guaranteed to be again a capsule. This mechanism is not possible in previous work [1, 10, 12. The type systems of Gordon et al. [15] and Pony [11] lack borrowed references in the formalization, but could support the variant where the scanner is isolated (capsule in our teminology) without losing isolation of the scanner or customer (the scanner could remain isolated throughout, relying on their support for dispatching appropriate methods on isolated references without explicit unpacking).

In the former example, explicit capsule opening and recovery take place inside the method body. Consider the following alternative version:

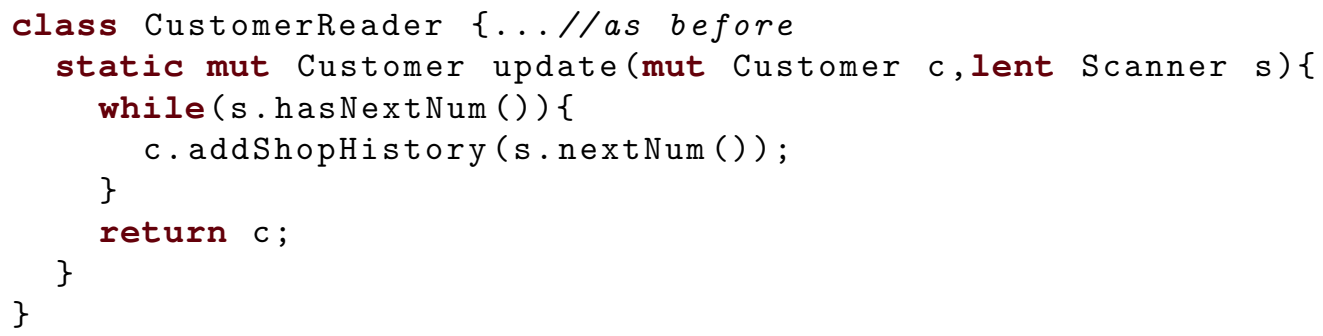


This method takes now a mut object and returns another mut one, as it usually happens in imperative programming. Surprisingly enough, this method turns out to be just a more flexible version of the former one. Indeed:

- If called on mut data, then it returns mut data, while a call of the former method was ill-typed.

- If called on capsule data, then capsule opening implicitly takes place during method call execution, and recovery can be achieved for the method call expression, returning a capsule as for the former method.

That is, recovery happens on the client side ${ }^{11}$. However, a client does not need to worry about this, and can simply call the method. In a sense, this version of update is polymorphic: it can be used as either mut $\rightarrow$ mut or as capsule $\rightarrow$ capsule.

This pattern can be used in combination with function composition. That is, a chain of mut $\rightarrow$ mut methods can be called, and, if we start from a capsule, we will get a capsule in the end. As shown in our example, these methods can have additional (non mut) parameters.

Moreover, this method can be trasparently used as lent $\rightarrow$ lent. That is, if called on lent data, then it returns lent data, by applying rule (T-Swap) to the method call expression. Again this can be extended to chains of methods which may have additional (non mut) parameters.

Finally, we show the code of the Scanner itself, and how swapping can be used to update the fields of a lent scanner in a safe way.

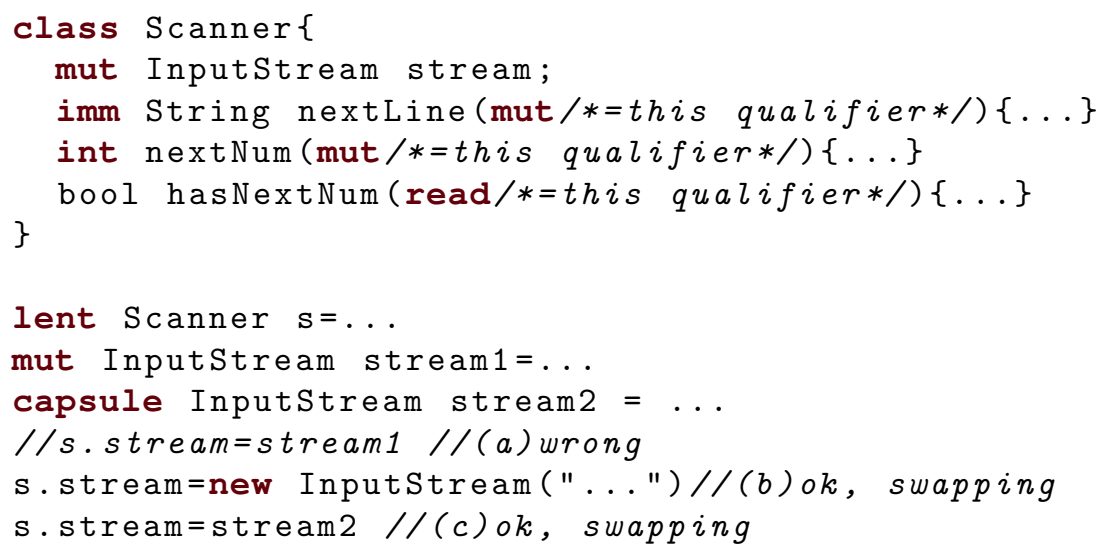

In our type system, a lent reference can be regarded as mutable if all the mutable references are regarded as lent, as formally modeled by rule (T-SwAP). This can be trivially applied to line (b), where $\mathrm{s}$ is the only free variable, and to line (c), where the other free variable is declared capsule. In line (a), instead, swapping would assign a lent type to stream1.

\footnotetext{
${ }^{11}$ The type systems of Gordon et al. 15] and Pony [11] could support a variant analogously to the case above.
} 
The this qualifiers reflect the fact that the first two methods modify, whereas the third only reads, the scanner's state. Note that, as in the previous example, the first two methods can be safely applied to lent scanners by swapping (in this case the result type, being not mutable, remains the same). Note also that such methods (as any method with no parameters and non mutable result type) could be equivalently have this qualifier lent, since, intuitively, no aliasing can be introduced for this (formally, we can apply rule (T-SwAP) to the method body). On the other hand, the first two methods can be invoked on lent scanners by by applying rule (T-SWAP) to the method call expression.

Readable and immutable references. We illustrate now the read and imm qualifiers by the example of a Person class with a list of friends.

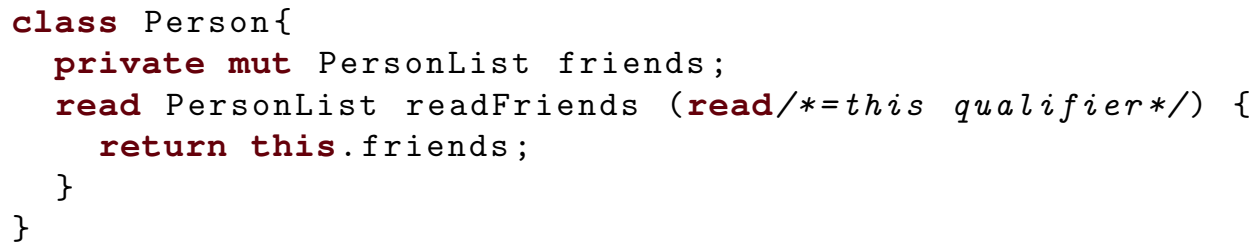

To give access to the private field, we declare a method which is like a usual getter, except that it returns a read PersonList reference. In this way, a client can only read the list of friends obtained through an invocation person. readFriends(), with person of class Person. Note that, since the this qualifier of the method is read, which is the top of the subtyping hierarchy, it can be invoked whichever is the qualifier of person. Moreover, in the case person is imm, we get an imm PersonList back, by recovering immutability for the read result. Indeed, in this case, we can apply rule (т-імм) to the invocation person.readFriends (), since the only free variable person is immutable, so no variable needs to be restricted. This is another case where the method is polymorphic: it can be used as either read $\rightarrow$ read or as imm $\rightarrow$ imm, and a client does not need to worry about, and can simply call the method.

Assume now that we want, instead, to give permission to a client to modify the list of friends. In this case, we can declare a getter method with different type annotations:

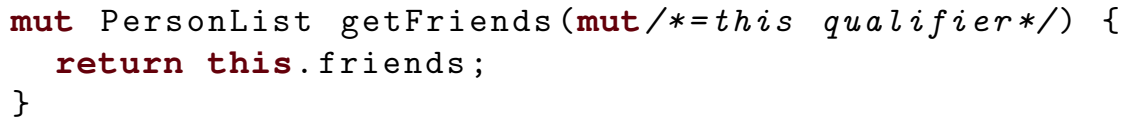

This method takes a mutable Person and returns a mutable PersonList reference. Hence, it cannot be invoked on read or imm objects. However, this mut getter can be invoked on a lent person: in person.getFriends() the only free variable person can be seen as mut. The result of the method is turned in lent PersonList by the (T-SWAP) rule (formally, swapping the singleton lent group person with the empty set).

Our approach forces the programmer to explicitly choose either read or mut getters. Other works permits the developer to use either multiple getters 
or polymorphic type qualifiers, for instance the type annotation PolyRead of Javari 29] allows one to keep a single method, providing an additional design choice for programmers. However, we argue that forcing programmers to consider the two operations as logically different can be a simpler and more explicit programming pattern. (In Section 7 we discuss also a third variant, with return type lent.) In a language supporting many levels of visibility (like protected, package, friend, ...) a programmer may choose a restricted visibility for mut getters and a more permissive visibility for read getters. Collection classes also can declare read and mut getters, as in the following example.

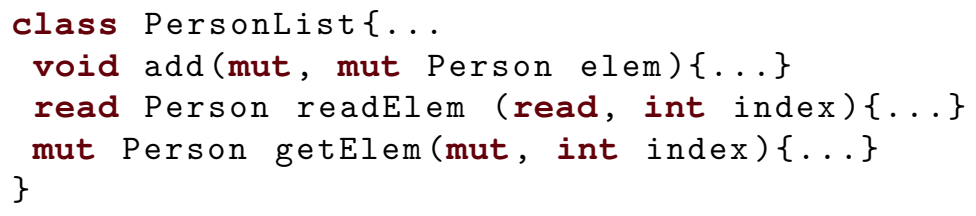

Finally, we show how we can create mutable objects, mutate them for a while, and then recover their immutabiity:

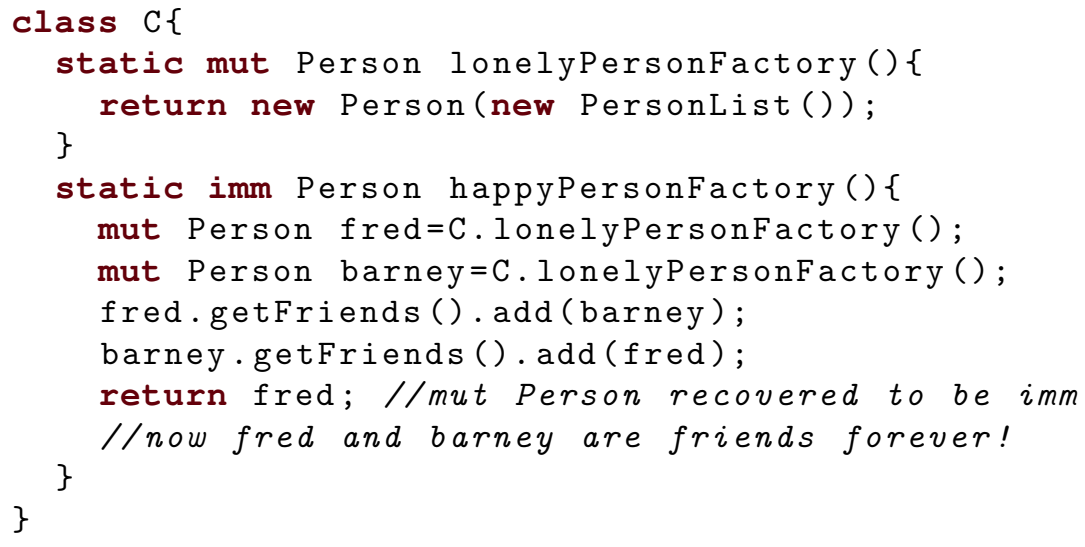

Here lonelyPersonFactory() creates lonely Persons, that have no friends. However, there is still hope, since they are mutable: happyPersonFactory creates two lonely people, fred and barney, and makes them friends. Then the function returns fred (and, indirectly, also barney that is now in the reachable object graph of fred). The function body does not use any free variable, so we can recover the capsule property of the result, hence return it as imm.

Qualifiers are deep. Note that recovery work since qualifiers have a deep/full interpretation, in the sense that they are propagated to the whole reachable object graph. In a shallow interpretation, instead, it is possible, for instance, to reach a mutable object from an immutable object. The advantage of such interpretation is that libraries can declare strong intentions in a coherent and uniform way, independently of the concrete representation of the user input (that, with the use of interfaces, could be unknown to the library). On the other side, providing (only) deep/full qualifiers means that we do not offer any language support for (as an example) an immutable list of mutable objects. 
Nested recovery and groups. We conclude the section by an example showing that recoveries can be nested, and, to ensure that all are safe, distinct lent groups must be kept. Consider the following code, where implementation of A is omitted to emphasize that only type information provided by qualifiers is significant.

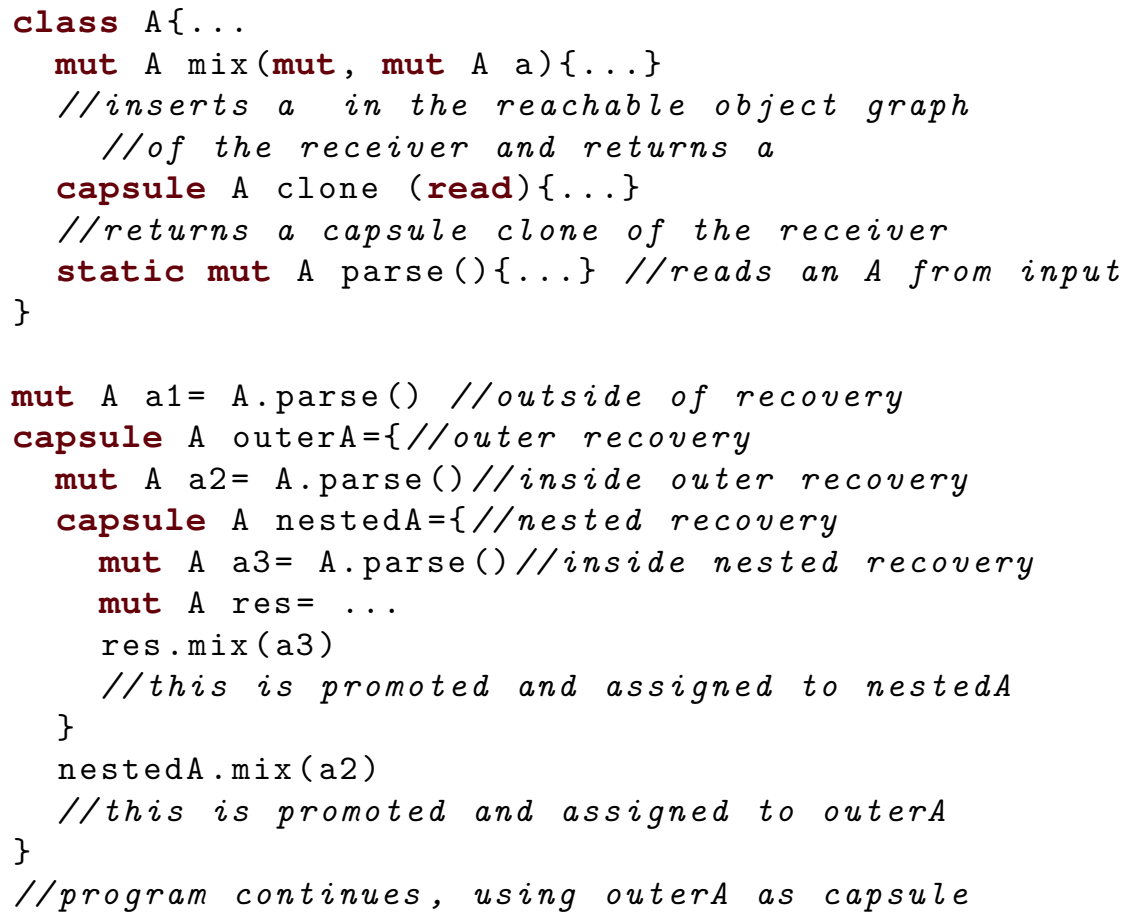

The question is, what can we write instead of the dots, and why. Clearly, (1) a3 is allowed, since the result of the inner block will be only connected to a local reference, while (2) a1 and a2 are not, since it will be connected to an external mutable reference. However, (3) a1.clone() and a2.clone() are allowed, since the method clone returns a capsule. In the same way, (4) a2 . mix (a2).clone() is allowed, as well as a1.mix(a1).clone().

However, when we start mixing different variables, things become trickier. For example, (5) a2.mix(a1).clone() is not well-typed in our type system. Indeed, even though, thanks to cloning, mixing a2 and a1 does not compromise the capsule well-formedness of nestedA (that is, the nested recovery can be safely applied), the fact that a2 and a1 are mixed could compromise the capsule well-formedness of outerA when outerA is computed (that is, the outer recovery would be unsafe).

In summary, mixing as lent groups introduced for different recoveries must be avoided. Rule (T-SwaP) swaps one group with another, thus keeping them distinct.

This last example illustrates many of the differences w.r.t. the type system proposed in [15, whose notion of recovery is less expressive. Their system allows 


\begin{tabular}{|clll|}
\hline$e$ & $::=x|v \cdot f| v \cdot m(v s)|v \cdot f=v|$ new $C(v s) \mid\{d s v\}$ & expression \\
$d$ & $::=T x=e ;$ & variable declaration \\
$T$ & $::=\mu C$ & type \\
$\mu$ & $::=\operatorname{mut} \mid$ imm $\mid$ capsule $\mid$ lent $\mid$ read & type qualifier \\
& & & \\
$u, v$ & $:=x \mid$ new $C(v s) \mid\{d v s v\}$ & value \\
$d v$ & $::=T x=r v ;$ & \\
$r v$ & $::=\operatorname{new} C(x s)|\{d v s x\}|\{d v s$ new $C(x s)\}$ & evaluated declaration \\
\end{tabular}

Figure 10: Simplified syntax and values

(1), and rejects (2) and (5), as ours. However, they conservatively rejects (3) and (4), since the flow is not tracked at a fine enough granularity. Depending on the concrete application, programmers may need to work around the limitations of [15] by reordering local variables, introducing stricter type qualifiers or, in general, re-factoring their code. However, there may be cases where there is no possible reordering.

\section{Calculus}

The calculus has a simplified syntax where we assume that, except from right-hand sides of declarations, subterms of a compound expression are only values. This simplification can be easily obtained by a (type-driven) translation of the syntax of Figure 1 generating for each subterm which is not a value a local declaration of the appropriate type.

Simplified syntax and values are defined in Figure 10. Syntax of evaluated declarations and right-values from Figure 1 is reported for reader's convenience.

A value is either a variable (reference), or a constructor invocation, or a value enclosed in a local store.

We denote by $\mathrm{FV}(e)$ the set of the free variables of expression $e$ (the standard formal definition is in Appendix A).

We assume the following well-formedness constraints on expressions:

1. In a block $\left\{T_{1} x_{1}=e_{1} ; \ldots T_{n} x_{n}=e_{n} ; v\right\}$, mutual recursion, that is, $x_{j} \in$ $\mathrm{FV}\left(e_{i}\right)$ and $x_{i} \in \mathrm{FV}\left(e_{j}\right)$, is only allowed if both declarations are evaluated declarations, formally defined in Figure 10. Hence, as expected, cyclic stores are allowed, such as

\{mut $C$ y=new $C(x)$; mut $C x=$ new $C(y) ; x\}$

\{mut $C \mathrm{x}=$ new $\mathrm{C}(\mathrm{x}) ; \mathrm{x}$ \}

whereas cyclic expressions such as

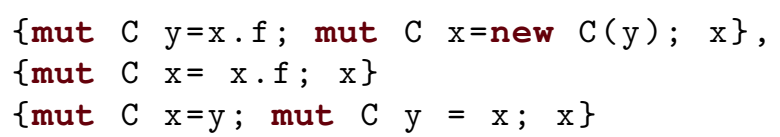


are ill-formed. Allowing general recursion would require a sophisticated type system, as in [26], but this is not the focus of this paper.

2. As already mentioned in Section 3, variables of capsule types can occur at most once in their scope. This is simply a syntactic constraint, that is, we do not deal with linear types and the resultant context splitting, or flow-sensitive typing judgments. For instance, the following expression, which clearly violates the intent of the capsule and immutable qualifiers, is ill-formed:

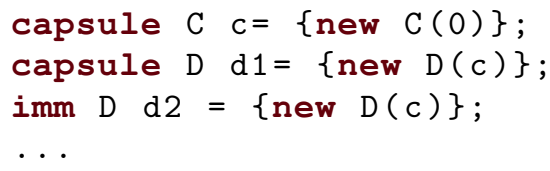

Note that a capsule variable is not yet determined to become mutable or immutable when it is declared, and that determination is made at the time of its unique occurrence.

Evaluated declarations associate a right-value to a variable, which plays the role of a reference. Hence, they correspond to the store in conventional models of imperative languages. In Figure 11 we define well-formed right-values and store.

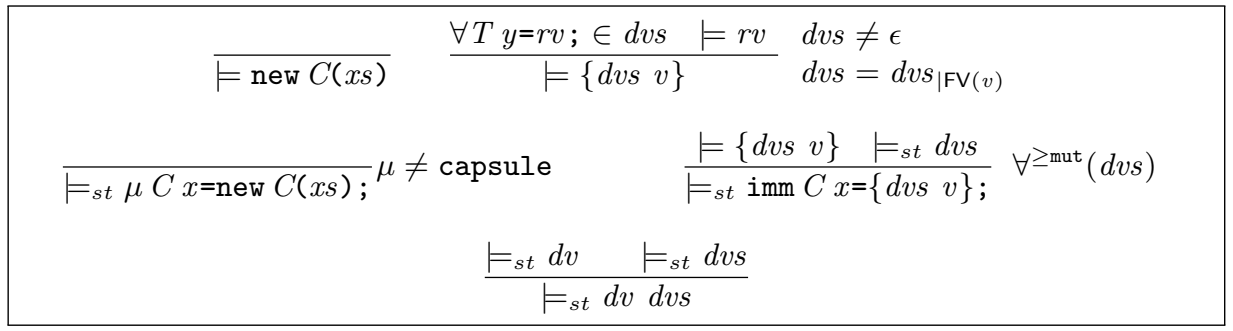

Figure 11: Well-formed right-values and store

For a sequence of declarations $d s=T_{1} x_{1}=e_{1} ; \ldots T_{n} x_{n}=e_{n}$; , and a set of variables $X$, we write $X \stackrel{d s}{\longrightarrow} x$ if $x$ is (transitively) connected to $X$ through $d s$. This relation is inductively defined as follows:

$$
\begin{gathered}
X \stackrel{d s}{\longrightarrow} x \text { if } x \in X \\
X \stackrel{d s}{\longrightarrow} x \text { if } x \in \mathrm{FV}\left(e_{i}\right) \text {, for some } i \in 1 . . n \text {, and } X \stackrel{d s}{\longrightarrow} x_{i} .
\end{gathered}
$$

The subsequence $d s_{\mid X}$ of the declarations that are (transitively) used by $X$ is defined by: for all $i \in 1 . . n, T_{i} x_{i}=e_{i} ; \in d s_{\mid X}$ if $X \stackrel{d s}{\longrightarrow} x_{i}$.

We write $\forall \geq$ mut $(d v s)$ if, for each $\mu C x=r v$; $\in d v s, \mu \geq$ mut, and $\forall^{\text {imm }}(d v s)$ if, for each $\mu C x=r v ; \in d v s, \mu=$ imm.

Rules in the first line define well-formed right-values. They state that a rightvalue should not contain garbage and useless blocks, and that, in case it is a block, the right-hand sides of declarations are, in turn, well-formed right-values. 
Rules in the second and third line define well-formed evaluated declarations or stores. The first rule states that a right-value which is an object state can be associated to any reference which is not capsule. The second rule states that a right-value which is a block can only be associated to an imm reference, its local store should not contain imm references, and, in addition to be a well-formed right-value, the block should contain a well-formed local store. The last rule states that a (non empty) sequence of evaluated declarations is a well-formed store if each one is a well-formed.

These rules altogether imply that in a well-formed store:

- There are no capsule references. Indeed, capsule declarations are "temporary", that is, are expected to be consumed as soon as their right-hand side has been evaluated, as will be formalized by reduction rule (CAPsuleeum) in Figure 14.

- There are at most two levels, that is, the right-value of an imm reference can contain in turn a local store of non imm references. Indeed, additional levels can safely be "flattened", as will be formalized by reduction rules (мит-моvе) and (тмм-моvе) in Figure 14.

For instance, assuming that class $\mathrm{C}$ has two mut fields of class D and one imm of class C, and class D has an integer field, the following is a store:

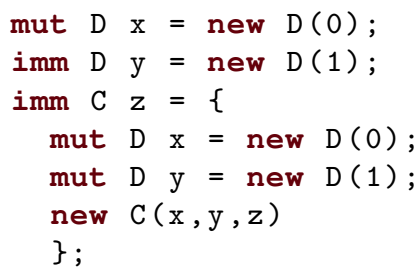

Note that mutable variables in the local store of $\mathbf{z}$ are not visible from the outside. This models in a natural way the fact that the portion of store denoted by $\mathbf{z}$ is indeed immutable

Expressions are equivalent modulo the congruence $\cong$ defined by the rules of Figure 12.

$$
\begin{gathered}
\text { (alpha) }\left\{d s \text { T } x=e ; d s^{\prime} v\right\} \cong\left\{d s[y / x] T y=e[y / x] ; d s^{\prime}[y / x] v[y / x]\right\} \\
\text { (REORDer) }\left\{d s d v d s^{\prime} v\right\} \cong\left\{d v d s d s^{\prime} v\right\}
\end{gathered}
$$

Figure 12: Congruence on expressions

Rule (ацрна) is the usual $\alpha$-conversion. We write $e\left[e^{\prime} / x\right]$ for the expression obtained by replacing all (free) occurrences of $x$ in $e$ by $e^{\prime}$ (the standard formal definition is in the Appendix). The condition $x, y \notin \operatorname{dom}\left(d s d s^{\prime}\right)$ is implicit by 
well-formedness of blocks. Rule (REORDER) states that we can move evaluated declarations in an arbitrary order. On the other hand, $d s$ and $d s^{\prime}$ cannot be swapped, since this could change the order of side effects.

Values are equivalent modulo the congruence $\cong$ defined by the rules of Figure 13 By rule (NEw) we can assume that arguments of a constructor invocation

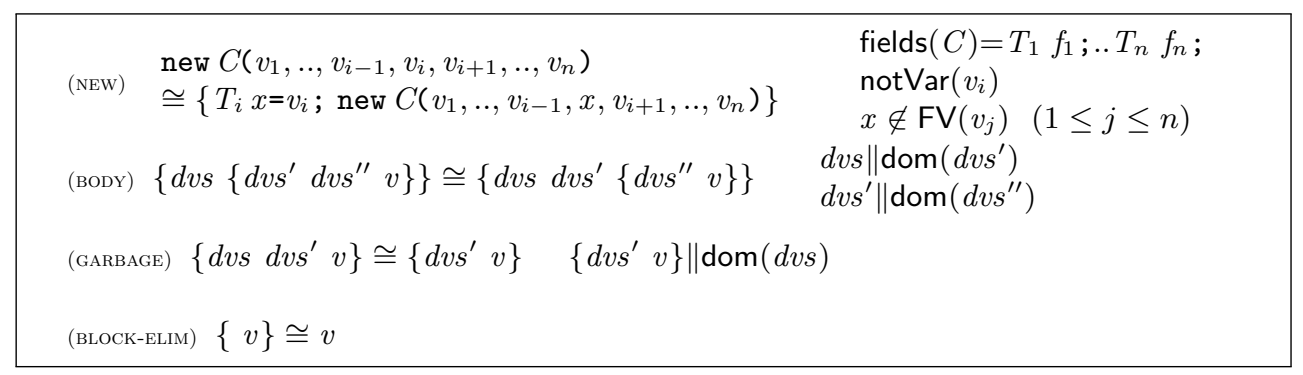

Figure 13: Congruence on values

are references, by introducing local declarations of the appropriate type. The notation $\operatorname{not} \operatorname{Var}(v)$ means that $v$ is not a variable. The following three rules deal with block values. By rule (воду) we can move a sequence of evaluated declarations from a block to the directly enclosing block, and conversely. The notation $e \| x s$ means that free variables in $e$ are not captured by $x s$, formally: $\mathrm{FV}(e) \cap x s=\emptyset$, and analogously for $d v s \| x s$. The two side conditions ensure that moving the declarations $d v s^{\prime}$ does not cause either scope extrusion or capture of free variables. More precisely: the first condition prevents capturing with $d v s^{\prime}$ some free variables of the enclosing block, whereas the second prevents moving outside a declaration in $d v s^{\prime}$ which depends on local variables of the inner block (declared in $d v s^{\prime \prime}$ ). The first side condition can be satisfied by $\alpha$-conversion of the inner block, whereas the second cannot. By rule (Garbage) we can remove useless local store from a block. Finally, rule (вьоск-ецім) states the obvious fact that a block with no declarations is equivalent to its body.

Congruence preserves types. We have to assume that the congruent expressions be typable, since rule (GARBAGE) eliminates declarations from blocks that without this assumption could be not typable.

Proposition 1. Let $\Gamma ; x s s ; y s \vdash e: T$. If $e \cong e^{\prime}$ and for some $\Gamma^{\prime}, x s s^{\prime}, y s^{\prime}$ and $T^{\prime}$ we have that $\Gamma^{\prime} ; x s s^{\prime} ; y s^{\prime} \vdash e^{\prime}: T^{\prime}$, then $\Gamma ; x s s ; y s \vdash e^{\prime}: T$

Proof By cases on the congruence rule used.

Let a well-formed value be either a variable or a well-formed right-value. We can show that any value is congruent to a well-formed value.

Proposition 2. Let $v$ be a value, then either $v \cong x$ for some variable $x$, or $v \cong r v$ for some right-value rv such that $=r v$.

Proof By induction on values using the congruence rule of Figure 13 . 
The reduction relation is defined by the rules in Figure 14, where $\mathcal{E}$ is an evaluation context defined by:

$$
\mathcal{E}::=[] \mid\{\text { dvs } T x=\mathcal{E} ; \text { ds } v\}
$$

In this definition, and in the following we assume that the metavariables $v, r v$, $d v$ and $d v s$ denote values, right-values, evaluated declarations, and stores which are well-formed. The assumption on $v$ and $r v$ can be achieved by Proposition 2 .

To lighten notations, here and in what follows we sometimes use the wildcard - when a metavariable is not significant.

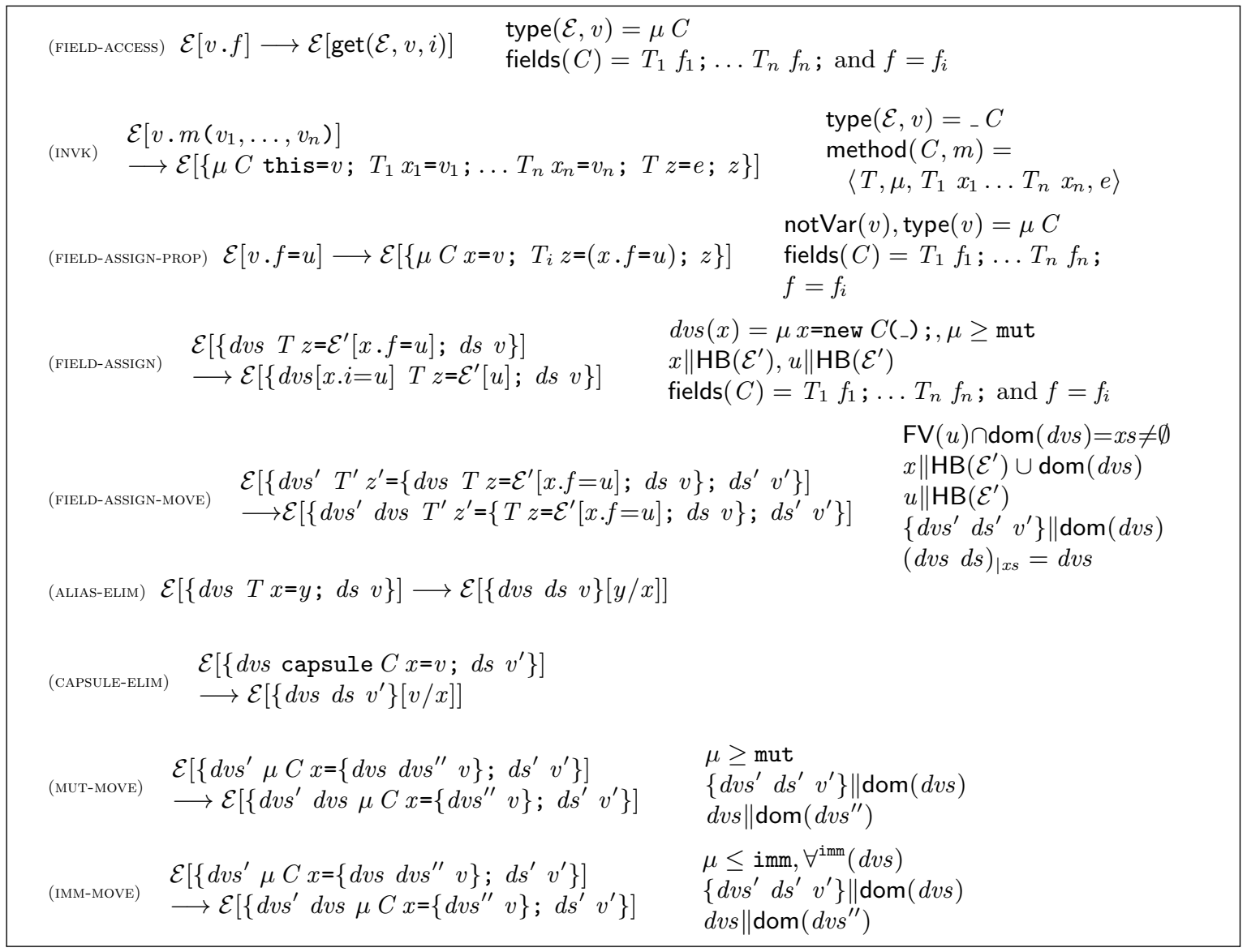

Figure 14: Reduction rules

In rule (FIELD-ACCESS), the type $\mu C$ of the receiver $v$ in the context is found, fields of the class $C$ are retrieved from the class table, it is checked that $f$ is actually the name of a field of $C$, say, the $i$-th field, and the field access is reduced to the $i$-th field of the receiver.

The auxiliary functions type and get extract the type, and the $i$-th field, respectively, of a value in a context (only needed when the value is a reference). 
In the definitions, by Proposition 2, we assume that values are either references or right-values.

$$
\begin{aligned}
& \operatorname{type}(\mathcal{E}, x)=T \text { if } \operatorname{dec}(\mathcal{E}, x)=T x=_{-} ; \\
& \operatorname{type}(\mathcal{E}, r v)=\operatorname{type}(r v) \\
& \text { type }(\text { new } C(x s))=\operatorname{type}(\{d v s \text { new } C(x s)\})=\operatorname{mut} C \\
& \operatorname{type}(\{d v s x\})=T \text { if } d v s(x)=T x_{-} ; \\
& \operatorname{get}(\mathcal{E}, x, i)=\operatorname{get}(r v, i) \text { if } \operatorname{dec}(\mathcal{E}, x)={ }_{-} x=r v ; \\
& \operatorname{get}(\mathcal{E}, r v, i)=\operatorname{get}(r v, i) \\
& \operatorname{get}\left(\text { new } C\left(x_{1}, \ldots, x_{n}\right), i\right)=x_{i} \\
& \operatorname{get}\left(\left\{d v s \text { new } C\left(x_{1}, \ldots, x_{n}\right)\right\}, i\right)=\left\{d v s x_{i}\right\} \\
& \operatorname{get}(\{d v s x\}, i)=\{d v s v\} \text { if } d v s(x)={ }_{-} x=r v ; \text { and get }(r v, i)=v \\
& \operatorname{dec}\left(\left\{d v s T_{-}=\mathcal{E} ; d s v\right\}, x\right)= \begin{cases}\operatorname{dec}(\mathcal{E}, x) & \text { if } \operatorname{dec}(\mathcal{E}, x) \text { defined, otherwise } \\
d v s(x) & \text { if } d v s(x) \text { defined }\end{cases}
\end{aligned}
$$

Note that a field access $x . f$, if $x$ has qualifier $\geq$ mut, always returns a reference, since the right-value of $x$ is necessarily an object state. If $x$ is immutable, instead, the field access could return a (copy of) the value stored in the field. This duplication preserves the expected semantics in the case of an immutable reference $x$, whereas it would be wrong for a mutable reference, since a modification of the object denoted by $x$ is expected to affect $x . f$ as well, and conversely.

For instance, given the value:

$$
v=\{\text { mut } \mathrm{C} \mathrm{x}=\text { new } \mathrm{C}(\mathrm{x}, \mathrm{y}, \mathrm{z}) \text {; mut } \mathrm{D} \mathrm{y}=\text { new } \mathrm{D}(0) \text {; new } \mathrm{C}(\mathrm{x}, \mathrm{y}, \mathrm{z})\}
$$

we have:

1. get $(v, 1)=$ mut $\mathrm{C} \mathrm{x}=$ new $\mathrm{C}(\mathrm{x}, \mathrm{y}, \mathrm{z}) ;$ mut $\mathrm{D} \mathrm{y}=$ new $\mathrm{D}(0) ; \mathrm{x}\}$

2. get $(v, 2)=$ mut $\mathrm{C} \mathrm{x}=$ new $\mathrm{C}(\mathrm{x}, \mathrm{y}, \mathrm{z})$; mut $\mathrm{D} \mathrm{y}=$ new $\mathrm{D}(0) ; \mathrm{y}\}$

3. get $(v, 3)=$ mut $\mathrm{C} \mathrm{x}=$ new $\mathrm{C}(\mathrm{x}, \mathrm{y}, \mathrm{z})$; mut $\mathrm{D} \mathrm{y}=$ new $\mathrm{D}(0) ; \mathrm{z}$ \}

where 1 is a well-formed value, 2 is congruent by rule (GARBAGE) to the wellformed value \{mut $\mathrm{D} y=$ new $\mathrm{D}(0) \mathrm{y}$ \}, and 3 is congruent by rules (Garbage) and (вLоск-еLiм), to the well-formed value $\mathbf{z}$.

If the value $v$ above is the right-value of a reference, then such reference is necessarily imm. In this case, $v$ was expected to be a capsule, the variable $z$ should be declared imm in the enclosing context.

In rule (INvK), the class $C$ of the receiver $v$ is found, and method $m$ of $C$ is retrieved from the class table. The call is reduced to a block where declarations of the appropriate type for this, the parameters, and the result are initialized with the receiver, the arguments, and the method body, respectively. The last declaration, variable $z$, is needed to preserve the simplified syntax.

Rule (FIELD-ASSign-PRop) handles the case of a field assignment where the receiver is not a reference (denoted notVar $(v)$ ). In this case, a local reference initialized with the receiver is introduced, and the field access is propagated to such reference.

In rule (FIELD-ASSIGN), given a field assignment where the receiver is a reference $x$, the first enclosing declaration for $x$ is found (the side condition $x \| \mathrm{HB}\left(\mathcal{E}^{\prime}\right)$ 
ensures that it is actually the first), it is checked that the qualifier of the type of $x$ is $\geq$ mut, and fields of the class $C$ of $x$ are retrieved from the class table. If $f$ is the name of a field of $C$, say, the $i$-th, then the $i$-th field of the right-value of $x$ is updated to $u$, which is also the result of the field assignment. We write $\operatorname{HB}(\mathcal{E})$ for the hole binders of $\mathcal{E}$, that is, the variables declared in blocks enclosing the context hole (the standard formal definition is in the Appendix) and $d v s[x . i=u]$ for the sequence of evaluated declarations obtained from dvs by replacing the $i$-th field of the right-value of $x$ with $u$ (the obvious formal definition is omitted).

The side condition $u \| \mathrm{HB}\left(\mathcal{E}^{\prime}\right)$, requiring that there are no inner declarations for some free variable in $u$, prevents scope extrusion. For instance, without this side condition, the term

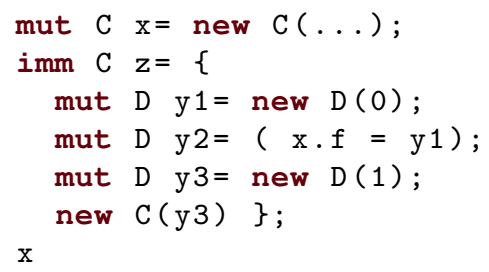

would erroneously reduce to

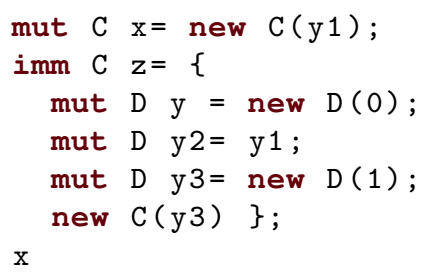

Thanks to the side condition, instead, rule (FIELD-Assign) is not applicable. However, rule (FIELD-ASSIGN-Move) can be applied.

Rule (FIELD-ASSign-move) moves store out of a block when this is needed to safely perform field-assignment (that is, to avoid scope extrusion). In this rule, given a field assignment of shape $x . f=u$, the first enclosing block containing (evaluated) declarations for some free variables $x s$ of $u$ is found (the side condition $u \| \operatorname{HB}\left(\mathcal{E}^{\prime}\right)$ ensures that it is actually the first). If a declaration for $x$ can only be found in an outer scope (side condition $x \| \operatorname{HB}\left(\mathcal{E}^{\prime}\right) \cup \operatorname{dom}(d v s)$ ), then the store formed by the $x s$ references, together with all the other references they (recursively) depend on (last side condition) is moved to the directly enclosing block.

For the example above, by applying rule (FIELD-ASSIGN-move) we get:

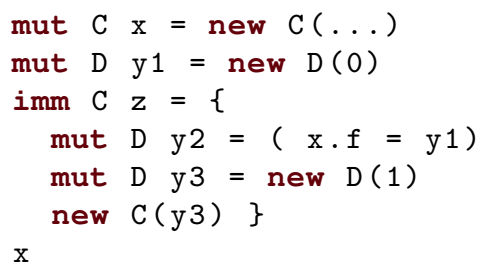


Now, rule (FIeLD-ASsign) can be safely applied to the term. In general, we may need to apply rule (FIELD-ASSIGN-MOve) many times before reaching the declaration of $x$.

The remaining rules handle blocks.

The first two rules eliminate a single declaration of shape $T x=v$; which is not well-formed store.

In rule (ALIAS-ELIM), a reference $x$ which is initialized as an alia: ${ }^{12}$ of another reference $y$ is eliminated by replacing all its occurrences.

In rule (CAPSULE-ELim), a capsule reference $x$ is eliminated by replacing the occurrence of $x$ (unique by the well-formedness constraint) by its value. Note that, in rule (ALIAS-ELIM), $y$ cannot be a capsule reference (since we would have applied rule (CAPSULE-ELIM) before), hence duplication of capsule references cannot be introduced, and well-formedness is preserved.

Rule (мит-моvе) moves store out of a block associated to a reference with qualifier $\geq$ mut. This is always safe, provided that no variables of the outer scope are captured (second side condition, which can be obtained by $\alpha$-renaming), and that the moved declarations do not refer to other declarations of the inner block (last side condition).

Rule (тмм-моve) moves store out of a block associated to a reference with qualifier $\leq$ imm. In this case, this is only safe for a store of imm references. The same side conditions of the previous rule are needed.

\section{Type safety and properties of the type system}

In this section we present the results. We first give a characterisation of the values in terms of the properties of their free variables, which correspond to their reachable graph. Then we show the soundness of the type system for the operational semantics, and finally we formalize the expected behaviour of capsule and immutable references.

In the following, we denote by $\mathcal{D}: \Delta \vdash e: T$ a derivation tree for the judgement $\Delta \vdash e: T$. Moreover, we call the rules where expressions in the premises are the direct subterms of the expression in the consequent, structural; the others, that is, (T-CAPSULE), (T-IMm), (T-SWAP), (T-UNRST), and (T-SUB), non-structural.

\subsection{Canonical Forms}

In a type derivation, given a construct, in addition to the corresponding structural rule we can have applications of non-structural rules. In this section we first present some results exploring the effect of non-structural rules on the lent groups and the mutable groups of variables, and on the modifier derived for the expression, then we give an inversion lemma for blocks (the construct which is relevant for the analysis of right-values), and finally we present the Canonical Form theorem with its proof.

\footnotetext{
${ }^{12} \mathrm{An}$ analogous rule would handle variables of primitive types in an extension of the calculus including such types.
} 
Given a type judgement $\Gamma$; xss $[x s] ; y s \vdash e: \mu C$, application of non-structural rules can only modify the lent groups and the mutable group by swapping, hence leading to a permutation $x s s^{\prime} x s$ of $x s s$ xs. In other terms, the equivalence relation on $\operatorname{dom}^{\text {mut }}(\Gamma)$ they induce is preserved, as the following lemma shows.

Lemma 3 (Non-structural rules). If $\mathcal{D}: \Gamma$; xss $[x s]$; ys $\vdash e: \mu C$, then there is a sub-derivation $\mathcal{D}^{\prime}$ of $\mathcal{D}$ such that $\mathcal{D}^{\prime}: \Gamma ; x s s^{\prime}\left[x s^{\prime}\right] ; y s^{\prime} \vdash e: \mu^{\prime} C$ ends with the application of a structural rule, xss $x s=x s s^{\prime} x s^{\prime}$, and $\mu \neq i m m, \mu \neq$ capsule implies $\mu^{\prime} \leq \mu$.

Proof The proof is in Appendix B.

The application of non-structural rules is finalized to the recovery of capsule and immutable properties as expressed by the following lemma.

Lemma 4. Let $\mathcal{D}: \Gamma ; x s s ; y s \vdash e: \mu C$.

1. If $\mu=$ mut, then the last rule applied in $\mathcal{D}$ cannot be (T-SWAP) or (T-UNRST) or (T-CAPSUle) or (T-IMM).

2. If $\mu=$ imm or $\mu=$ capsule and the last rule applied in $\mathcal{D}$ is (T-SWAP) or (T-UnRst), then $\Gamma ; x s s^{\prime} ; y s^{\prime} \vdash e: \mu C$, for some $x s s^{\prime}$ and $y s^{\prime}$.

\section{Proof}

1. Rule (T-UNRST) is only applicable when the type derived for the expression in the premise of the rule, $\mu^{\prime} C$, is such that $\mu^{\prime} \leq$ imm. For the rule (T-SWAP), when the type derived for the expression in the premise of the rule is mut $C$, then the the type of the expression in the consequent is lent $C$. Moreover, the two recovery rules do not derive types with the mut modifier.

2. Immediate.

From the previous lemma we derive that, if $\Gamma$; xss; ys $\vdash e: \mu C$ with $\mu \nsupseteq$ lent, then without loss of generality we can assume that the last rule applied in the derivation is either a structural rule followed by a (Т-suв), or a recovery rule in case $\mu=$ capsule or $\mu=$ imm. Moreover, rules (T-SWAP) and (T-UnRst) cannot be used to derive the premise of rule (T-CAPSULE).

If we can derive a type for a variable, then such type depends on the type assignment and the lent groups (if the variable is restricted no type can be derived). If the variable is declared with modifier imm or capsule, then its type depends only on the type assignment.

Lemma 5. If $\Gamma$; xss; ys $\vdash x: T$ then $\Gamma(x) \leq T$. Moreover, if $T=\mu C$ with $\mu \leq i m m$, then $\Gamma ; x s s^{\prime} ; y s^{\prime} \vdash x: T$ for all $x s s^{\prime}$ and $y s^{\prime}$ such that $\vdash \Gamma ; x s s^{\prime} ; y s^{\prime}$.

Proof The proof is in Appendix B 
The block construct is central to our recovery technique. New variable are defined, and the lent and mutable groups of the free variables of the block may be modified by the introduction of the newly defined variables. However, as for the non-structural rules, the equivalence relation between the mutable variables induced by the partition determined by the lent and mutable groups is preserved.

We first define a judgment asserting when a declaration of a variable is well typed and then give a lemma relating the type judgement for a block with the type judgements for the expressions associated to its local variables by the declarations and for its body. The expression associated with a variable declared with modifier lent has to be well typed taking as mutable the variable in its group, whereas the others have the same mutable group of the body of the expression. This expresses the fact that variables declared with modifier lent should not be connected to the result of the block (its body).

Definition 6. Define $\Gamma$; $x s s[x s] ; y s \vdash \mu C x=e$; OK by:

- $\Gamma$; xss $[x s] ; y s \vdash e: \Gamma(x)$ if $\mu \neq$ lent

- $\Gamma ; x s s^{\prime}\left[x s^{\prime}\right] ; y s \vdash e: \Gamma(x)$ if $\mu=$ lent and $x s s x s=x s s^{\prime} x s^{\prime}$ where $x \in x s^{\prime}$.

$\Gamma ; x s s[x s] ; y s \vdash d s \mathrm{OK}$ if for all $d \in d s, \Gamma ; x s s[x s] ; y s \vdash d \mathrm{OK}$.

Lemma 7 (Inversion for blocks). If $\Gamma ; x s s[x s] ; y s \vdash\{d s v\}: \mu C$, then for some $x s s^{\prime}$ and $x s^{\prime}$

1. $\Gamma\left[\Gamma_{d s}\right] ; x s s^{\prime}\left[x s^{\prime}\right] ; y s^{\prime} \vdash d s \mathrm{OK}$

2. $\Gamma\left[\Gamma_{d s}\right] ; x s s^{\prime}\left[x s^{\prime}\right] ; y s^{\prime} \vdash v: \mu^{\prime} C$

3. (xss $x s) \backslash \operatorname{dom}\left(\Gamma_{d s}\right) \sqsubseteq x s s^{\prime} x s^{\prime}$ and

4. $\mu \neq$ imm and $\mu \neq$ capsule implies $\mu^{\prime} \leq \mu$.

Proof The proof is in Appendix B

The two following lemmas characterize the shape of mut right-values. For constructor values we also have that if the mutable variables are restricted then all fields must have immutable types.

Lemma 8 (Constructor value). Let fields $(C)=\mu_{1} C_{1} f_{1} ; \ldots \mu_{n} C_{n} f_{n}$; .

1. If $\Gamma$; xss $[x s] ;$ ys $\vdash$ new $C\left(z_{1}, \ldots, z_{n}\right):$ mut $C$, then: for all $i \in 1 . . n$, if $\mu_{i}=$ mut, then $z_{i} \in x s$, otherwise $\Gamma ; x s s[x s] ; y s \vdash z_{i}: \mu C_{i}$ with $\mu \leq i m m$.

2. If $\Gamma ; x s s[\emptyset] ; \operatorname{dom}^{\geq m u t}(\Gamma) \vdash$ new $C\left(z_{1}, \ldots, z_{n}\right):$ read $C$, then: for all $i \in 1 . . n$ we have that $\Gamma$; xss $[x s]$; ys $\vdash z_{i}: \mu C_{i}$ with $\mu \leq i m m$.

Proof The proof is in Appendix B

In case a right-value is a block, the fact that all the declared variables are connected to the body of the block implies that the modifiers of all the variables must be either mut or $\leq$ imm. 
Lemma 9 (Block value). If $\Gamma$; xss $[x s] ; y s \vdash\{d v s w\}:$ mut $C$, where $d v s=$ $\mu_{1} C_{1} z_{1}=r v_{1} ; \cdots \mu_{n} C_{n} z_{n}=r v_{n} ;$, then

1. $\Gamma\left[\Gamma_{d v s}\right] ; x s s^{\prime}\left[x s^{\prime}\right] ; y s \vdash w:$ mut $C$,

2. $\Gamma\left[\Gamma_{d v s}\right] ; x s s^{\prime}\left[x s^{\prime}\right] ; y s \vdash r v_{i}:$ mut $C_{i} \forall i \in 1 . . n$

3. for all $z \in F V(\{d v s w\})$, if $\Gamma(z)=$ mut $D$, then $z \in x s$, otherwise $\Gamma$; xss $[x s]$; ys $\vdash$ $z: \mu C$ with $\mu \leq i m m$.

Proof The proof is in Appendix B

As usual typing depends only on the free variable of expressions, as the following weakening lemma states.

Lemma 10 (Weakening). Let $\operatorname{dom}\left(\Gamma^{\prime}\right) \cap F V(e)=\emptyset$ and $\vdash \Gamma\left[\Gamma^{\prime}\right] ; x s s ; y s$.

$\Gamma\left[\Gamma^{\prime}\right] ; x s s ; y s \vdash e: T$ if and only if $\Gamma ; x s s \backslash \operatorname{dom}\left(\Gamma^{\prime}\right) ; y s \backslash \operatorname{dom}\left(\Gamma^{\prime}\right) \vdash e: T$.

Proof By induction on derivations.

The Canonical Forms theorem describes constraints on the free variables and the extracted type of well-typed right-values. In particular, capsule and imm right-values can contain only capsule or imm references, and mut right-values cannot contain lent or read references. Moreover, the type extracted from capsule and mut right-values is necessarily mut.

Theorem 11 (Canonical Forms). If $\Gamma$; xss $[x s] ; y s \vdash r v: \mu_{-}$, and $y \in F V(r v)$, then:

1. if $\mu=$ capsule, then $\Gamma$; xss $[x s] ; y s \vdash y:$ imm $_{-}$, and type $(r v)=m u t_{-}$

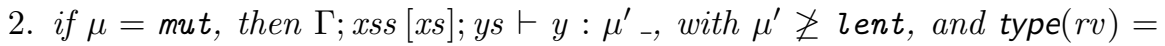
mut

3. if $\mu=i m m$, then $\Gamma$; xss $[x s] ; y s \vdash y: i m m_{-}$

4. if $\mu=$ lent, then $\Gamma ; x s s[x s] ; y s \vdash y: r_{\text {read }}$, and type $(r v) \leq$ lent $_{-}$

5. if $\mu=$ read, then $\Gamma$; xss $[x s]$; ys $\vdash y:$ read $_{-}$

Proof By structural induction on $r v$ and by cases on $\mu$.

Let $r v=$ new $C\left(x_{1}, \ldots, x_{n}\right)$ and fields $(C)=\mu_{1} C_{1} f_{1} ; \ldots \mu_{n} C_{n} f_{n} ;$. By definition

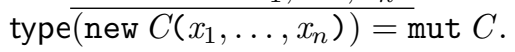

If $\mu=$ capsule, from Lemma 42 we can assume that the last rule applied in the derivation is (T-CAPSULE):

$$
\frac{\Gamma ; x s s x s[\emptyset] ; y s \vdash \text { new } C(z s): \text { mut } C}{\Gamma ; x s s[x s] ; y s \vdash \text { new } C(z s): \text { capsule } C}(\text { T-CAPsule })
$$

From Lemma 8.1, since the current mutable group is empty, $\forall i \in 1 . . n$ we have that $\Gamma ; x s s[x s] ; y s \vdash x_{i}: \operatorname{imm} C_{i}$. 
If $\mu=$ mut, from rule (T-NEw), $\Gamma$; xss $[x s] ; y s \vdash$ new $C\left(x_{1}, \ldots, x_{n}\right):$ mut $C$. Therefore, from Lemma 8.1 we get the result.

Let $\mu=$ imm. From Lemma 42 we can assume that the last rule applied in the derivation is either (т-suв), or (т-гмм). In the first case $\Gamma$; xss $[x s]$; ys $\vdash$ new $C(z s)$ : capsule $C$, so the proof for $\mu=$ capsule applies. In the second, we have

$$
\frac{\mathcal{D}: \Gamma ; x s s x s[\emptyset] ; \operatorname{dom}^{\geq \operatorname{mut}}(\Gamma) \vdash \operatorname{new} C(z s): \operatorname{read} C}{\Gamma ; x s s[x s] ; y s \vdash \text { new } C(z s): \operatorname{imm} C}(\text { (--імм) }
$$

From Lemma 8 , we get the result.

Let $\mu=$ lent or $\mu=$ read. Then to derive a type for new $C(x s)$ we have to apply (T-NEW) followed by (T-SUB), so the result is obvious.

Let $r v=\{d v s w\}$ where $d v s$ is $T_{1} y_{1}=r v_{1} ; \ldots T_{n} y_{n}=r v_{n}$; , and $w=$ new $C(x s)$ or $w=x$.

If $\mu=$ capsule, then, from Lemma 42 we can assume that the last rule applied in the derivation is (T-CAPSULE):

$$
\frac{\Gamma ; x s s x s[\emptyset] ; y s \vdash\left\{d v s_{-}\right\}: \operatorname{mut} C}{\Gamma ; x s s[x s] ; y s \vdash\left\{d v s_{-}\right\}: \text {capsule } C}(\text { T-CAPsuLe })
$$

From Lemma 9, since the current mutable group is empty, $\forall i \in 1$..n we have that $\Gamma$; xss $[x s] ; y s \vdash x_{i}: \operatorname{imm} C_{i}$.

If $\mu=$ mut, Lemma 41 implies that the last rule of the type derivation is either an application of (т-SUB), or of (т-BLоск). In the first case the proof for $\mu=$ capsule applies. In the second case, we have

$$
\Gamma ; x s s ; y s \vdash\left\{d v s_{-}\right\}: \text {mut } C
$$

From Lemma 9 we get the result.

If $\mu=\mathrm{imm}$, from Lemma 42 we can assume that the last rule applied is either (TsuB) or (Т-Iмм). If the rule applied is (т-suв), then $\Gamma ; x s s ; y s \vdash\left\{d v s_{-}\right\}$: capsule $C$, and the proof for $\mu=$ capsule applies.

Let the last rule applied in $\mathcal{D}^{\prime}$ be (т-Iмm), i.e.,

$$
\frac{\mathcal{D}: \Gamma ; x s s \text { xs }[\emptyset] ; \operatorname{dom}^{\geq \operatorname{mut}}(\Gamma) \vdash\left\{d v s_{-}\right\}: \operatorname{read} C}{\Gamma ; x s s[x s] ; y s \vdash\left\{d v s_{-}\right\}: \operatorname{imm} C}(\text { т-гмм) }
$$

From Lemma 7, since we may assume that in the derivation $\mathcal{D}$ the sub-derivation that ends with rule (т-вLоск) is not the antecedent of rule (T-UnRst) (otherwise the block would already have type imm $C$ ) we have that

(a) $\Gamma\left[\Gamma_{d v s}\right] ; x s s^{\prime}\left[x s^{\prime}\right] ; y s^{\prime} \vdash_{-}: \mu^{\prime} C$

(b) $\Gamma\left[\Gamma_{d v s}\right] ; x s s^{\prime}\left[x s^{\prime}\right] ; y s^{\prime} \vdash \mu_{i} C_{i} z_{i}=r v_{i}$; OK $\forall i \in 1 . . n$ 
(c) (xss $x s) \backslash \operatorname{dom}\left(\Gamma_{d v s}\right) \sqsubseteq x s s^{\prime} x s^{\prime}$ and

(d) $\operatorname{dom}^{\geq \operatorname{mut}}(\Gamma) \backslash \operatorname{dom}(d v s)=y s^{\prime}$

If $y \in \mathrm{FV}\left(\left\{d v s_{-}\right\}\right)$, then either $y \in \mathrm{FV}(-) \backslash \operatorname{dom}(d v s)$ or $y \in \mathrm{FV}\left(r v_{i}\right) \backslash \operatorname{dom}(d v s)$ for some $i \in 1$..n.

If $y \in \mathrm{FV}\left({ }_{-}\right) \backslash \operatorname{dom}(d v s)$ and $y \in \operatorname{dom}^{\geq \operatorname{mut}}(\Gamma)$, from (d) $y \in y s^{\prime}$. In order to apply rule (T-VAR) to derive a type for $y$ we would have to apply rule (T-UNRST). Therefore $\Gamma\left[\Gamma_{d v s}\right](y)=\mu D$ with $\mu \leq$ imm. (Rule (T-UNRST) cannot follow (T-NEw).) So $y \notin \operatorname{dom}^{\geq \text {mut }}(\Gamma)$, and $\Gamma(y)=\mu D$ with $\mu \leq \mathrm{imm}$.

If for some $i \in 1 . . n, y \in \mathrm{FV}\left(r v_{i}\right) \backslash \operatorname{dom}(d v s)$ and $y \in \operatorname{dom}^{\geq \operatorname{mut}}(\Gamma)$, let $\mathcal{D}$ : $\Gamma\left[\Gamma_{d v s}\right] ; x s s_{i}\left[x s_{i}\right] ; y s^{\prime} \vdash r v_{i}: \mu_{i} C_{i}$. Since $y \in \operatorname{dom}^{\geq \operatorname{mut}}(\Gamma)$ there is a subderivation $\mathcal{D}^{\prime}$ of $\mathcal{D}$, such that $\mathcal{D}^{\prime}: \Gamma\left[\Gamma_{d v s}\right] ; x s s_{i}^{\prime}\left[x s_{i}^{\prime}\right] ; y s^{\prime} \vdash v^{\prime}: \mu^{\prime} C^{\prime}$, the last rule applied is (T-UNRST), and $y \in \mathrm{FV}\left(v^{\prime}\right)$, i.e.,

$$
\frac{\Gamma\left[\Gamma_{d v s}\right] ; x s s_{i}^{\prime}\left[x s_{i}^{\prime}\right] ; \emptyset \vdash v^{\prime}: \text { imm } C^{\prime}}{\Gamma\left[\Gamma_{d v s}\right] ; x s s_{i}^{\prime}\left[x s_{i}^{\prime}\right] ; y s^{\prime} \vdash v^{\prime}: \operatorname{imm} C^{\prime}}(\text { т-UNRST })
$$

If $v^{\prime}=y$ then $\Gamma\left[\Gamma_{d v s}\right](y)=\mu D$ with $\mu \leq$ imm, therefore also $\Gamma(y)=\mu D$ with $\mu \leq \mathrm{imm}$, which is impossible since $y \in \operatorname{dom}^{\geq \operatorname{mut}}(\Gamma)$.

If $v^{\prime}=r v^{\prime}$ for some $r v^{\prime}$, by induction hypothesis on $r v^{\prime}$, we have that $\Gamma\left[\Gamma_{d v s}\right] ; x s s_{i}^{\prime}\left[x s_{i}^{\prime}\right] ; \emptyset \vdash$ $y: \mu D$ where $\mu \leq \mathrm{imm}$. Since $y \notin \operatorname{dom}(d v s)$ and $\mu \leq \mathrm{imm}$, by Lemma 10 and Lemma 5. also $\Gamma(y)=\mu D$ with $\mu \leq \mathrm{imm}$, which is again impossible since $y \in \operatorname{dom}^{-{ }^{-m u t}}(\Gamma)$.

Therefore, for all $y \in \mathrm{FV}\left(\left\{d v s_{-}\right\}\right)$, we have that $\Gamma$; xss; ys $\vdash y$ : imm $D$ with $\mu \leq \mathrm{imm}$.

If $\mu=$ lent or $\mu=$ read, then $y$ must be such that $\Gamma$; xss; ys $\vdash y: \mu^{\prime} D$, so also $\Gamma ; \overline{x s s} ; y s \vdash y: \operatorname{read} D$.

Moreover, if $\mu=$ lent and $\Gamma$; xss; ys $\vdash\{d v s w\}:$ lent $C$, in case $w=$ new $C\left(x s^{\prime}\right)$, we have that type $(\{d v s w\})=\operatorname{mut} C$, and if $w=x$, so $x=y_{1}$, must be that $T_{1}=\mu_{1} C$ with $\mu_{1} \leq$ lent.

Note that there are no constraints for read right-values (by subtyping any welltyped right-value is also read), and no constraints on free variables for lent right-values. Moreover, note that a right-value with only $\leq$ imm free variables is imm regardless of its extracted type, since we can apply immutability recovery.

\subsection{Soundness of the type system for the operational semantics}

As usual soundness is proved by proving that typability is preserved by reduction, "subject reduction", and that well-typed expressions are either values of reduce, "progress". The subject reduction result, in our system, is particularly relevant, since invariants on the store are expressed at the syntactic level by the modifiers assigned to the expression and by the lent and mutable groups used in the typing judgement. Preserving typability of expressions means not only enforcing the properties expressed by modifiers, but also preserving the relationship between variables expressed by the lent and mutable groups.

To identify subexpressions of expressions we define general contexts $\mathcal{G}$ by: 


$$
\begin{aligned}
& \mathcal{G}::= {[]|\mathcal{G} \cdot f| \mathcal{G} \cdot m(v s)\left|v \cdot m\left(v s \mathcal{G} v s^{\prime}\right)\right| \mathcal{G} \cdot f=v \mid v \cdot f=\mathcal{G} } \\
& \mid \text { new } C\left(v s \mathcal{G} v s^{\prime}\right)\left|\left\{d s T x=\mathcal{G} ; d s^{\prime} v\right\}\right|\{d s \mathcal{G}\}
\end{aligned}
$$

Given a general context $\mathcal{G}, \Gamma_{\mathcal{G}}$ is defined by:

- $\Gamma_{[]}=\emptyset$

- $\Gamma_{\mathcal{G} . f}=\Gamma_{\mathcal{G} . m(v s)}=\Gamma_{v \cdot m\left(v s \mathcal{G} v s^{\prime}\right)}=\Gamma_{\mathcal{G} . f=v}=\Gamma_{v . f=\mathcal{G}}=\Gamma_{\text {new } C\left(v s \mathcal{G} v s^{\prime}\right)}=\Gamma_{\mathcal{G}}$

- $\left.\Gamma_{\{d s} T x=\mathcal{G} ; d s^{\prime} v\right\}=\Gamma_{d s}, \Gamma_{d s^{\prime}}, x: T\left[\Gamma_{\mathcal{G}}\right]$

- $\left.\Gamma_{\{d s} \mathcal{G}\right\}=\Gamma_{d s}\left[\Gamma_{\mathcal{G}}\right]$

$\Gamma_{\mathcal{E}}$ is defined similarly. We also use lent $\operatorname{Vars}(\mathcal{G})$ and lent $\operatorname{Vars}(\mathcal{E})$ with the obvious meaning.

The following lemma expresses the property that subexpressions of welltyped expressions are themselves well-typed in a type context that may contain more variables, introduced by inner blocks. The equivalence relation on the variable of the expressions induced by the partition determined by the lent and mutable groups is preserved. However, more variables may be added to existing groups, there could be new groups, and due to rule (T-SwaP), the mutable group of the subexpression may be different from the one on the whole expression.

Lemma 12 (Context). If $\mathcal{D}: \Gamma$; xss $[x s]$; ys $\vdash e: T, e=\mathcal{G}\left[e^{\prime}\right]$ and $\mathcal{D}^{\prime}$ : $\Gamma\left[\Gamma_{\mathcal{G}}\right] ; x s s^{\prime}\left[x s^{\prime}\right] ; y s^{\prime} \vdash e^{\prime}: T^{\prime}$ is a sub-derivation of $\mathcal{D}$, then

1. - (xss xs $) \backslash \operatorname{dom}\left(\Gamma_{\mathcal{G}}\right) \sqsubseteq x s s^{\prime} x s^{\prime}$,

- $\operatorname{dom}\left(\Gamma_{\mathcal{G}}\right)=\emptyset$ implies xss $x s=x s s^{\prime} x s^{\prime}$, and

- we may assume that the last rule applied in $\mathcal{D}^{\prime}$ is a structural rule;

2. if $e^{\prime \prime}$ is such that $\mathcal{D}^{\prime \prime}: \Gamma\left[\Gamma_{\mathcal{G}}\right] ; x s s^{\prime} ; y s^{\prime} \vdash e^{\prime \prime}: T^{\prime}$, then substituting $\mathcal{D}^{\prime \prime}$ with $\mathcal{D}^{\prime}$ in $\mathcal{D}$ we get a derivation for $\Gamma ; x s s[x s] ; y s \vdash \mathcal{E}\left[e^{\prime}\right]: T$.

Proof The proof is in Appendix B

Note that, if $\mathcal{G}$ is [ ] or [ ].f or [ ].m(vs) or $v \cdot m\left(v s\right.$ [] $\left.v s^{\prime}\right)$ or [ ] $f=v$ or $v \cdot f=[$ ] or new $C\left(v s[] v s^{\prime}\right)$, then $\operatorname{dom}(\mathcal{G})=\emptyset$ and Lemma 12 implies Lemma 3.

Lemma 13 (Field access). Let fields $(C)=\mu_{i} C_{i} f_{1} ; \ldots \mu_{n} C_{n} f_{n}$;.

1. If $\Gamma ; x s s[x s] ; y s \vdash$ new $C(x s) . f_{i}: \mu C_{i}$ where the last rule applied is (T-FIELDACCEss), then $\Gamma ; x s s ; y s \vdash x_{i}: \mu C_{i}$.

2. If Aux $\Gamma ; x s s ; x s \vdash y s: r v . f_{i} T$ where the last rule applied in $\mathcal{D}$ is (T-FIELDACCEss), $\Gamma ; x s s[x s] ; y s \vdash \operatorname{get}(r v, i): T$.

3. If $\Gamma ; x s s[x s] ; y s \vdash r v . f_{i}:$ imm $C_{i}$, then for all $x s s^{\prime}, y s^{\prime}$ such that $\vdash \Gamma ; x s s^{\prime} ; y s^{\prime}$ we have that $\Gamma ; x s s^{\prime}\left[x s^{\prime}\right] ; y s^{\prime} \vdash \operatorname{get}(r v, i):$ imm $C_{i}$.

Proof The proof is in Appendix B.

Lemma 14 (Field assign). Let $\mathcal{D}: \Gamma ; x s s[x s] ; y s \vdash x . f=u: T$, and the last rule applied in $\mathcal{D}$ be (T-FIELD-ASSIGN). Then, $x \in x$ s and for all $y \in F V(u)$ such that $\Gamma ; x s s ; y s \vdash y: \mu_{-}$with $\mu \geq$ mut we have that $y \in x s$. 
Proof The proof is in Appendix B.

The following theorem asserts that reduction preserves typability of expressions. The theorem is proved by cases on the reduction rule used. Here we give the proof for the two difficult cases, which are the application of (FIELD-Access), and (Field-Assign-Move). In the appendix we present the proof for other interesting cases, that use similar techinques. The difficulty with the proof of subject reduction, for (Field-ACCEss), is the non standard semantics of the construct, that replaces the "field access expression" with the "value of the field". The "value of the field" must be given the same type it had in the context of the declaration of the variable. For the rule (FIeld-Assign-Move), the difficult cases are when declarations are moved outside a block to which the recovery rules are applied. As highlighted in the proof, in these cases, the derivation contained application of the rule (T-SWAP) (if it was a capsule) and (T-UNRST) (if it was imm), which are still applicable after moving the declarations.

Theorem 15 (Subject Reduction). Let $\vdash e: T$, and $e \longrightarrow e^{\prime}$. Then $\vdash e^{\prime}: T$.

Proof Let $\vdash e: T$. If $e \longrightarrow e^{\prime}$, then one of the rules of Figure 14 was applied. Here we prove the result for the most interesting rules: (FIELD-ACCESS), and (FIELDAssign-move, whose proofs embodies the techniques used. In the Section Appendix B we present two other interesting cases (Field-Assign), and (мuт-move), which are easier, but still interesting.

Consider rule (FIELD-ACCESS). In this case

(1) $e=\mathcal{E}[v \cdot f]$, and

(2) $e^{\prime}=\mathcal{E}[\operatorname{get}(\mathcal{E}, v, i)]$,

where fields $(C)=\mu_{i} C_{i} f_{1} ; \ldots \mu_{n} C_{n} f_{n}$; and $f=f_{i}$.

From (1) and Lemma 121 for some $T^{\prime}$, xss and ys we have that

(3) $\Gamma_{\mathcal{E}} ; x s s[x s] ; y s \vdash v \cdot f: T^{\prime}$ and

(4) the last rule appled in the derivation is (FIELD-ACCESS).

From Proposition 2 either $v \cong z$ for some $z$ or $v \cong r v$ for some right-value $r v$ such that $=r v$.

If $v \cong r v$, from (3), (4), Lemma 132 and $\operatorname{get}(\mathcal{E}, v, i)=\operatorname{get}(r v, i)$ we have that $\Gamma_{\mathcal{E}} ; x s s ; y s \vdash \operatorname{get}(r v, i): T^{\prime}$. Therefore, from Lemma 122 we derive $\vdash e^{\prime}: T$.

If $\underline{v \cong x}$, since get $(\mathcal{E}, x, i)$ is defined $e=\mathcal{E}^{\prime}\left[\left\{d s^{\prime} v^{\prime}\right\}\right]$ where

(a) $d s^{\prime}=d v s \mu_{x} C x=r v_{x} ; T_{z} z=\mathcal{E}_{z}[x . f] ; d s$

(b) $={ }_{s t} \mu_{x} C x=r v_{x}$;

(c) $x \| \mathrm{HB}\left(\mathcal{E}_{z}\right)$ and we may assume that $r v_{x} \| \mathrm{HB}\left(\mathcal{E}_{z}\right)$.

Let $\Gamma^{\prime}=\Gamma_{\mathcal{E}^{\prime}}\left[\Gamma_{d s^{\prime}}\right]$, from Lemmas 12 and 7 for some $x s s^{\prime}$ and $y s^{\prime}$

(d) $\Gamma^{\prime} ; x s s^{\prime}\left[x s^{\prime}\right] ; y s^{\prime} \vdash \mu_{x} C x=r v_{x}$; OK, i.e.,

i $\Gamma^{\prime} ; x s s^{\prime}\left[x s^{\prime}\right] ; y s^{\prime} \vdash r v_{x}: \Gamma^{\prime}(x)$ if $\mu_{x} \neq$ lent

ii $\Gamma^{\prime} ; x s s_{x}\left[x s_{x}\right] ; y s^{\prime} \vdash r v_{x}: \Gamma^{\prime}(x)$ if $\mu_{x}=$ lent and $x s s^{\prime} x s^{\prime}=x s s_{x} x s_{x}$ where $x \in x s_{x}$ 
(e) $\left(x s s^{\prime} x s^{\prime}\right) \backslash \operatorname{dom}\left(\Gamma_{\mathcal{E}_{z}}\right) \sqsubseteq x s s x s$.

From (3), (4) and rule (Field-Access) we derive that

(f) $\Gamma_{\mathcal{E}} ; x s s[x s] ; y s \vdash x: \mu^{\prime} C$ and

(g) $T^{\prime}=\mu C_{i}$ where: if either $\mu_{i}=$ imm or $\mu^{\prime}=\mathrm{imm}$, then $\mu=\mathrm{imm}$, else $\mu=\mu^{\prime}$.

Consider the case $T^{\prime}=\mathrm{imm} C_{i}$ : either $\mu^{\prime}=\mathrm{imm}$ or $\mu_{i}=\mathrm{imm}$.

If $\mu^{\prime}=\mathrm{imm}, \Gamma_{\mathcal{E}} ; x s s ; y s \vdash x: \mathrm{imm} C$. From Lemmas 5 and 10 we have that also $\Gamma^{\prime} ; x s s^{\prime} ; y s^{\prime} \vdash x$ : imm $C$, therefore from (d).i we derive that $\Gamma^{\prime} ; x s s^{\prime}\left[x s^{\prime}\right] ; y s^{\prime} \vdash$ $r v_{x}$ : imm $C$. Applying rule (Field-Access), $\Gamma^{\prime} ; x s s^{\prime} ; y s^{\prime} \vdash r v_{x} . f_{i}:$ imm $C_{i}$. From (c), (e), and Lemma 10 we have that also $\Gamma_{\mathcal{E}} ; x s s ; y s \vdash r v_{x} . f_{i}:$ imm $C_{i}$. From Lemma 132 , we get that $\Gamma_{\mathcal{E}} ; x s s ; y s \vdash \operatorname{get}\left(r v_{x}, i\right): \operatorname{imm} C_{i}$. Since get $(\mathcal{E}, x, i)=$ get $\left(r v_{x}, i\right)$, from Lemma 122 we derive $\vdash e^{\prime}: T$.

If $\mu_{i}=\mathrm{imm}$, from (d).i (or (d).ii) and rule (FIELD-ACCEss) we derive $\Gamma^{\prime} ; x s s^{\prime}\left[x s^{\prime}\right] ; y s^{\prime} \vdash$ $r v_{x} \cdot f_{i}:$ imm $C_{i}$ (or $\Gamma^{\prime} ; x s s_{x}\left[x s_{x}\right] ; y s^{\prime} \vdash r v_{x} . f_{i}:$ imm $C_{i}$ ). From (e), Lemma 133 and Lemma 10 we get $\Gamma_{\mathcal{E}} ; x s s ; y s \vdash \operatorname{get}\left(r v_{x}, i\right):$ imm $C_{i}$, which implies, as for the previous case, $\vdash e^{\prime}: T$.

Consider the case $T^{\prime}=\mu^{\prime} C_{i}$ where $\mu^{\prime} \neq \mathrm{imm}$ and $\mu_{i} \neq \mathrm{imm}$, and, since we do not allow forward references to unevaluated declarations, also $\mu^{\prime} \neq$ capsule. Therefore $\mu^{\prime} \geq$ mut and $\mu_{i}=$ mut. From $\mu^{\prime} \geq$ mut and (b) the declaration of $x$ is of the shape

(h) $\mu_{x} C x$ =new $C(x s)$; where $\mu_{x} \geq \operatorname{mut}$ and $\operatorname{get}(\mathcal{E}, x, i)=x_{i}$.

If $\mu_{x}=$ mut, then $\Gamma^{\prime}(x)=\operatorname{mut} C$. From (d).i we have $\Gamma^{\prime} ; x s s^{\prime}\left[x s^{\prime}\right] ; y s^{\prime} \vdash$ new $C(x s)$ : mut $C$ So $x \in x s^{\prime}$ and from Lemma 8 also $x_{i} \in x s^{\prime}$.

If $\mu_{x}=$ lent, then $\Gamma^{\prime}(x)=$ mut $C$. From (d).ii we have that $\Gamma^{\prime} ; x s s_{x}\left[x s_{x}\right] ; y s^{\prime} \vdash$ new $C(x s)$ : mut $C$ where $x \in x s_{x}$. Again from Lemma 8 also $x_{i} \in x s_{x}$.

From (e) and (c) we have that $x$ and $x_{i}$ are in the same group in xss xs. Therefore, $\Gamma_{\mathcal{E}} ; x s s[x s] ; y s \vdash x_{i}: T^{\prime}$, and from Lemma 12 , 2 we derive $\vdash e^{\prime}: T$.

Finally, if $\mu_{x}=\operatorname{read}$, then $\Gamma^{\prime}(x)=\operatorname{read} C$, and from (d).i and rule (FIeldAccess) we derive $\Gamma^{\prime} ; x s s^{\prime}\left[x s^{\prime}\right] ; y s^{\prime} \vdash r v_{x} . f_{i}: \operatorname{read} C_{i}$. From $\Gamma^{\prime} ; x s s^{\prime}\left[x s^{\prime}\right] ; y s^{\prime} \vdash x_{i}$ : read $C_{i}$. and Lemma 10 we get $\Gamma_{\mathcal{E}} ; x s s ; y s \vdash x_{i}: \operatorname{read} C_{i}$, which implies, as for the previous case, $\vdash e^{\prime}: T$.

Consider rule (FIELD-ASSIGN-MOVE). In this case

(1) $e=\mathcal{E}\left[\left\{d v s^{\prime} \mu C z^{\prime}=e_{1} ; d s^{\prime} v^{\prime}\right\}\right]$, and

(2) $e^{\prime}=\mathcal{E}\left[\left\{d v s^{\prime} \quad d v s \mu C z^{\prime}=e_{2} ; d s^{\prime} v^{\prime}\right\}\right]$,

where

- $e_{1}=\left\{d v s \mu_{z} C_{z} z=\mathcal{E}^{\prime}[x \cdot f=u] ; d s v\right\}$,

- $e_{2}=\left\{\mu_{z} C_{z} z=\mathcal{E}^{\prime}[x . f=u] ; d s v\right\}$,

fields $(C)=T_{1} f_{1} ; \ldots T_{n} f_{n}$; with $f=f_{i}$ and

(3) $\mathrm{FV}(u) \cap \operatorname{dom}(d v s)=z s \neq \emptyset$,

(4) $x\left\|\mathrm{HB}\left(\mathcal{E}^{\prime}\right) \cup \operatorname{dom}(d v s), u\right\| \mathrm{HB}\left(\mathcal{E}^{\prime}\right),\left\{d v s^{\prime} d v s v^{\prime}\right\} \| \operatorname{dom}(d v s)$, 
(5) $z^{\prime} \notin \operatorname{dom}(d v s)$ and $(d v s d s)_{\mid z s}=d v s$.

Moreover, since forward definitions are only allowed to evaluated declarations, we have that

(6) $z \notin \mathrm{FV}(d v s)$.

From $\vdash e: T$ and Lemma 121 we get that, for some $T_{b}, x s s^{\prime \prime}$, and $y s^{\prime \prime}$

$(*) \Gamma_{\mathcal{E}} ; x s s^{\prime \prime}\left[x s^{\prime \prime}\right] ; y s^{\prime \prime} \vdash\left\{d v s^{\prime} \mu_{z} C z^{\prime}=e_{1} ; d s^{\prime} v^{\prime}\right\}: T_{b}$.

Let $\Gamma=\Gamma_{d v s^{\prime}}, \Gamma_{d s^{\prime}}, z^{\prime}: T^{\prime}$ From (*) and Lemma 7 for some $T^{\prime}, \mu^{\prime}, x s s^{\prime}$ and $y s^{\prime}$

(A) $\Gamma_{\mathcal{E}}[\Gamma] ; x s s^{\prime}\left[x s^{\prime}\right] ; y s^{\prime} \vdash d v s^{\prime} d s^{\prime} \mathrm{OK}$,

(B) $\Gamma_{\mathcal{E}}[\Gamma] ; x s s^{\prime}\left[x s^{\prime}\right] ; y s^{\prime} \vdash \mu C z^{\prime}=e_{1}$; OK, i.e.

i $\Gamma_{\mathcal{E}}[\Gamma] ; x s s^{\prime}\left[x s^{\prime}\right] ; y s^{\prime} \vdash e_{1}: \Gamma_{\mathcal{E}}[\Gamma]\left(z^{\prime}\right)$ if $\mu \neq$ lent

ii $\Gamma_{\mathcal{E}}[\Gamma] ; x s s_{z^{\prime}}\left[x s_{z^{\prime}}\right] ; y s^{\prime} \vdash e_{1}: \Gamma_{\mathcal{E}}[\Gamma]\left(z^{\prime}\right)$ if $\mu=$ lent and $x s s^{\prime} x s^{\prime}=$ $x s s_{z^{\prime}} x s_{z^{\prime}}$ where $z^{\prime} \in x s_{z^{\prime}}$

(C) $\Gamma_{\mathcal{E}}[\Gamma] ; x s s^{\prime} ; y s^{\prime} \vdash v^{\prime}: T^{\prime}$ and

(D) $\left(x s s^{\prime \prime} x s^{\prime \prime}\right) \backslash \operatorname{dom}(\Gamma) \sqsubseteq x s s^{\prime} x s^{\prime}$.

If $\mu \geq$ mut, we can give a proof similar to the case of rule (мuт-моve). Therefore we can assume that $\mu=$ capsule or $\mu=$ imm.

In both cases $\mu \neq$ lent, so from (B).i we have that

(B1) $\Gamma_{\mathcal{E}}[\Gamma] ; x s s^{\prime}\left[x s^{\prime}\right] ; y s^{\prime} \vdash e_{1}: \mu C$

Consider first $\mu=$ capsule.

In this case, from Lemma 42 we can assume that the last rule applied in the derivation of $e_{1}$ is (T-CAPSULE):

$$
\frac{\Gamma_{\mathcal{E}}[\Gamma] ; x s s^{\prime} x s^{\prime}[\emptyset] ; y s^{\prime} \vdash e_{1}: \operatorname{mut} C}{\Gamma_{\mathcal{E}}[\Gamma] ; x s s^{\prime}\left[x s^{\prime}\right] ; y s^{\prime} \vdash e_{1}: \text { capsule } C}(\text { T-CAPsule })
$$

Let $\Gamma^{\prime}=\Gamma_{d v s}, \Gamma_{d s}, z: T$. From Lemma 7 , for some $x s s$ and $y s$ we have that

(a) $\Gamma_{\mathcal{E}}\left[\Gamma\left[\Gamma^{\prime}\right]\right] ; x s s[x s] ; y s \vdash d v s \mathrm{OK}$,

(b) $\Gamma_{\mathcal{E}}\left[\Gamma\left[\Gamma^{\prime}\right]\right] ; x s s[x s] ; y s \vdash d s \mathrm{OK}$,

(c) $\Gamma_{\mathcal{E}}\left[\Gamma\left[\Gamma^{\prime}\right]\right] ; x s s[x s] ; y s \vdash T_{z} z=\mathcal{E}^{\prime}[x . f=u]$; OK, i.e.

i. $\Gamma_{\mathcal{E}}\left[\Gamma\left[\Gamma^{\prime}\right]\right] ; x s s[x s] ; y s \vdash \mathcal{E}^{\prime}[x . f=u]: \Gamma_{\mathcal{E}}[\Gamma](z)$ if $\mu_{z} \neq$ lent

ii. $\Gamma_{\mathcal{E}}\left[\Gamma\left[\Gamma^{\prime}\right]\right] ; x s s_{z}\left[x s_{z}\right] ; y s^{\prime} \vdash \mathcal{E}^{\prime}[x \cdot f=u]: \Gamma_{\mathcal{E}}[\Gamma](z)$ if $\mu_{z}=$ lent and $x s s x s=$ $x s s_{z} x s_{z}$ where $z^{\prime} \in x s_{z^{\prime}}$

(d) $\Gamma_{\mathcal{E}}\left[\Gamma\left[\Gamma^{\prime}\right]\right] ; x s s[x s] ; y s \vdash v$ : mut $C$

(e) $\left(x s s^{\prime} x s^{\prime}\right) \backslash \operatorname{dom}\left(\Gamma^{\prime}\right) \sqsubseteq x s s$ xs.

From (c) and Lemma 121 we have that, for some $x s s_{x}$ and $y s_{x}$

- $\Gamma_{\mathcal{E}}\left[\Gamma\left[\Gamma^{\prime}\left[\Gamma_{\mathcal{E}^{\prime}}\right]\right]\right] ; x s s_{x}\left[x s_{x}\right] ; y s_{x} \vdash x . f=u: T_{i}$ 
From Lemma 14 we derive that $x \in x s_{x}$, and, if $Y=\left\{y \mid d v s(y)=\mu_{y-} y=_{-} ; \mu_{y} \geq\right.$ mut $\}$, then for all $y \in Y$ we have that $y \in x s_{x}$.

From (3), (4), $x \| \operatorname{dom}(d s)$ (forward references are only allowed to evaluated declarations), (e) and (D) we have that $\{x\} \cup Y$ is a subset of one of the groups in xss xs. (Note that, in the derivation of the judgement (c).i or (c).ii, there must be an application of rule (T-SwAP) to make $x s_{x}$ the mutable group, since $x$ is in one of the groups of $x s s x s$.) Define $x s s^{*}$ and $y s^{*}$ as follows. If there is $z s^{\prime} \in x s s^{\prime}$ such that $x \in z s^{\prime}$, then $x s s^{*}=\left(x s s^{\prime}-z s^{\prime}\right)\left(z s^{\prime} \cup Y\right)$, otherwise $x s s^{*}=x s s^{\prime}$. If $x \in y s^{\prime}$ then $y s^{*}=y s^{\prime} \cup Y$, otherwise $y s^{*}=y s^{\prime}$.

From (4), (A), (B), (C) and Lemma 10, we derive

(A1) $\Gamma_{\mathcal{E}}\left[\Gamma, \Gamma_{d v s}\right] ; x s s^{*}\left[x s^{*}\right] ; y s^{*} \vdash d v s^{\prime} d s^{\prime} \mathrm{OK}$,

(C1) $\Gamma_{\mathcal{E}}\left[\Gamma, \Gamma_{d v s}\right] ; x s s^{*}\left[x s^{*}\right] ; y s^{*} \vdash v^{\prime}: T^{\prime}$

(D1) $\left(x s s^{\prime} x s^{\prime}\right) \backslash \operatorname{dom}(d v s) \sqsubseteq x s s^{*} x s^{*}$ and $y s^{\prime} \backslash \operatorname{dom}(d v s)=y s^{*} \backslash \operatorname{dom}(d v s)$.

From (a), (4), and Lemma 10 we have

(a1) $\Gamma_{\mathcal{E}}\left[\Gamma, \Gamma_{d v s}\right] ; x s s^{*}\left[x s^{*}\right] ; y s^{*} \vdash d v s \mathrm{OK}$

From (4) and the fact that, for well-formedness of declarations $x \notin \operatorname{dom}(d v s)$, we derive that $\Gamma\left[\Gamma_{d v s}, \Gamma_{d s}, z^{\prime}: T^{\prime}\right]=\Gamma, \Gamma_{d v s}\left[\Gamma_{d s}, z^{\prime}: T^{\prime}\right]$.

From (b), (c), (d), (e) and rule (т-вцоск), we have that

- $\Gamma_{\mathcal{E}}\left[\Gamma, \Gamma_{d v s}\right] ; x s s^{\prime} x s^{\prime}[\emptyset] ; y s^{\prime} \vdash\left\{\mu_{z} C_{z} z=\mathcal{E}^{\prime}[x . f=u] ; d s v\right\}$ : mut $C$

and therefore applying rule (T-CAPsule) we derive $\Gamma_{\mathcal{E}}\left[\Gamma, \Gamma_{d v s}\right] ; x s s^{\prime}\left[x s^{\prime}\right] ; y s^{\prime} \vdash e_{2} \mathrm{OK}$. From (D1) and Lemma 10 we also have

(B2) $\Gamma_{\mathcal{E}}\left[\Gamma, \Gamma_{d v s}\right] ; x s s^{*}\left[x s^{*}\right] ; y s^{*} \vdash \mu_{z} C z^{\prime}=e_{2} ; \mathrm{OK}$.

From (A1), (a1), (B2), (C1), (D), and (D1), applying rule (т-вцоск), we derive

$$
\Gamma_{\mathcal{E}} ; x s s^{\prime \prime}\left[x s^{\prime \prime}\right] ; y s^{\prime \prime} \vdash\left\{d v s^{\prime} \text { dvs } \mu C z^{\prime}=e_{2} ; d s^{\prime} v^{\prime}\right\}: T_{b} .
$$

From Lemma 12 2, we obtain the result.

Consider now $\mu=$ imm.

If the typing is obtained from (T-CAPSULE) followed by (T-SUB) the result follows from the previous proof. If instead the last rule applied was (т-гмм), from Lemma 4.2

$$
\frac{\Gamma_{\mathcal{E}}[\Gamma] ; x s s^{\prime} x s^{\prime}[\emptyset] ; \operatorname{dom}{ }^{\text {mut }} \Gamma_{\mathcal{E}}[\Gamma] \vdash e_{1}: \operatorname{read} C}{\Gamma_{\mathcal{E}}[\Gamma] ; x s s^{\prime}\left[x s^{\prime}\right] ; y s^{\prime} \vdash e_{1}: \operatorname{imm} C}(\text { т-імм) }
$$

Let $\Gamma^{\prime}=\Gamma_{d v s}, \Gamma_{d s}, z^{\prime}: T^{\prime}$. From Lemma 7 and the fact that there is no application of (т-UNRST) in the derivation of $e_{1}$ (if there was, then the type derived for $e_{1}$ should be imm $C$ ), for some $x s s$, and $y s$

(a) $\Gamma_{\mathcal{E}}\left[\Gamma\left[\Gamma^{\prime}\right]\right] ; x s s[x s] ; y s \vdash d v s$ OK,

(b) $\Gamma_{\mathcal{E}}\left[\Gamma\left[\Gamma^{\prime}\right]\right] ; x s s[x s] ; y s \vdash d s$ OK,

(c) $\Gamma_{\mathcal{E}}\left[\Gamma\left[\Gamma^{\prime}\right]\right] ; x s s[x s] ; y s \vdash T_{z} z=\mathcal{E}^{\prime}[x \cdot f=u]$; OK, i.e. 
i. $\Gamma_{\mathcal{E}}\left[\Gamma\left[\Gamma^{\prime}\right]\right] ; x s s[x s] ; y s \vdash \mathcal{E}^{\prime}[x . f=u]: \Gamma_{\mathcal{E}}[\Gamma](z)$ if $\mu_{z} \neq$ lent

ii. $\Gamma_{\mathcal{E}}\left[\Gamma\left[\Gamma^{\prime}\right]\right] ; x s s_{z}\left[x s_{z}\right] ; y s \vdash \mathcal{E}^{\prime}[x . f=u]: \Gamma_{\mathcal{E}}[\Gamma](z)$ if $\mu_{z}=$ lent and $x s s x=$ $x s s_{z} x s_{z}$ where $z^{\prime} \in x s_{z^{\prime}}$

(d) $\Gamma_{\mathcal{E}}\left[\Gamma\left[\Gamma^{\prime}\right]\right] ; x s s[x s] ; y s \vdash v: \operatorname{read} C$

(e) $\left(x s s^{\prime} x s^{\prime}\right) \backslash \operatorname{dom}\left(\Gamma^{\prime}\right) \sqsubseteq x s s x s$

(f) $y s=\operatorname{dom}^{\geq \operatorname{mut}} \Gamma_{\mathcal{E}}[\Gamma] \backslash \operatorname{dom}\left(\Gamma^{\prime}\right)$

As before, we can assume that $x \| \operatorname{dom}(d s)$. From (c), (4), Lemma 12.1, and rule (T-Field-ASsign), we derive that $x \in \operatorname{dom}^{\text {mut }}\left(\Gamma_{\mathcal{E}}[\Gamma]\right)$. So $x \in \operatorname{dom}^{-{ }^{\text {mut }}} \Gamma_{\mathcal{E}}[\Gamma]$. Therefore, in the derivation of the judgement (c).i or (c).ii, there must be an application of rule (T-UNRST) to make possible the application of rule (T-VAR) to $x$. The proof of this case now proceeds as for the case of $\mu=$ capsule defining $x s s^{*}$ and $y s^{*}$ and proving that $e_{2}$ and then the resulting expression are typeable.

To prove progress, given an expression we need to find a rule that may be applied, and prove that its side conditions are verified. To match an expression with the left-side of a rule we define pre-redexes, $\rho$, by:

$$
\begin{aligned}
\rho::= & v \cdot f|v \cdot m(v s)| v \cdot f=v^{\prime} \\
& \mid\{d v s T x=v ; d s v\} \quad \models_{s t} d v s \wedge \forall_{s t} T x=v ;
\end{aligned}
$$

Any expression can be uniquely decomposed in a in a context filled a pre-redex.

Lemma 16 (Unique Decomposition). Let $e$ be an expression. Either $e \cong v$ where $v$ is well-formed, and if $v=\{d v s w\}$, then $\models_{s t} d v s$, or there are $\mathcal{E}$ and $\rho$ such that $e \cong \mathcal{E}[\rho]$.

Proof The proof is in Appendix B

The progress result is proved by structural induction on expressions. The interesting case is field assignment, in which we have to prove that one of the three rules may be applied.

Theorem 17 (Progress). Let $\vdash e: T$. Then either $e \cong v$ where $v$ is wellformed, and if $v=\{d v s w\}$, then $\models_{s t} d v s$, or $e \longrightarrow e^{\prime}$ for some $e^{\prime}$.

Proof Let $e$ be such that $\vdash e: T$ for some $T$, and for no $v$ we have that $e \cong v$ where $v$ is well-formed, and if $v=\{d v s w\}$, then $\models_{s t} d v s$. By Lemma 16 for some $\mathcal{E}$ and $\rho$ we have that $e \cong \mathcal{E}[\rho]$, and so $\vdash \mathcal{E}[\rho]: T$. From Lemma 12 , $\Gamma_{\mathcal{E}} ; x s s ; y s \vdash \rho: T^{\prime}$, for some $x s s, y s, T^{\prime}$.

Let $\rho=v . f$. From $\Gamma_{\mathcal{E}} ; x s s ; y s \vdash v . f: T^{\prime}$, we have that $\Gamma_{\mathcal{E}} ; x s s ; y s \vdash v:{ }_{-} C$ with fields $(C)=T_{1} f_{1} ; \ldots T_{n} f_{n}$; and $f=f_{i}$. Therefore type $(\mathcal{E}, v)={ }_{-} C$ and $\operatorname{get}(\mathcal{E}, v, i)$ is defined. So rule (FIELD-Access) is applicable.

Let $\rho=v \cdot m(v s)$, then rule (INvK) is applicable.

Let $\rho=v \cdot f=v^{\prime}$, and not $\operatorname{Var}(v)$. Then rule (FIELD-ASSIGN-Prop) is applicable.

Let $\overline{\rho=x . f=v}$. From from $\Gamma_{\mathcal{E}} ; x s s ; y s \vdash x . f=v: T^{\prime}$, we have that $\Gamma_{\mathcal{E}} ; x s s ; y s \vdash$ $x$ : mut $C$ with fields $(C)=T_{1} f_{1} ; \ldots T_{n} f_{n}$; and $f=f_{i}$. Since $x \in \operatorname{HB}(\mathcal{E})$, then $\mathcal{E}=\mathcal{E}_{x}\left[\left\{\begin{array}{lll}d v s & T y=\mathcal{E}^{\prime} ; d s & u\end{array}\right\}\right]$ for some $\mathcal{E}^{\prime}$ and $\mathcal{E}_{x}$ such that $d v s(x)=d v$ and 
$x \notin \mathrm{HB}\left(\mathcal{E}^{\prime}\right)$. From $\models$ st $d v$, we have that $d v=$ mut $C x=$ new $C(x s) ;$.

There are two cases: either $v \| \operatorname{HB}\left(\mathcal{E}^{\prime}\right)$, or for some $z \in \operatorname{FV}(v), z \in \operatorname{HB}\left(\mathcal{E}^{\prime}\right)$.

In the first case rule (FIELD-ASsign) is applicable.

In the second, let $\mathcal{E}^{\prime}=\mathcal{E}_{z}\left[\left\{d v s^{\prime} T^{\prime} y^{\prime}=\mathcal{E}^{\prime \prime} ; d s^{\prime} u^{\prime}\right\}\right]$ such that $v \| \operatorname{HB}\left(\mathcal{E}^{\prime \prime}\right)$, and $d v s^{\prime}(z)=d v^{\prime}$ and $z \notin \mathrm{HB}\left(\mathcal{E}^{\prime \prime}\right)$.

If $\mathcal{E}_{z}$ is [ ], then (Field-assign-move) is applicable to

$$
\mathcal{E}_{x}\left[\left\{d v s \quad T y=\left\{d v s^{\prime} T^{\prime} y^{\prime}=\mathcal{E}^{\prime \prime}[x . f=v] ; d s^{\prime} u^{\prime}\right\} ; d s u\right\}\right] .
$$

Otherwise, $\mathcal{E}_{z}=\mathcal{E}_{z}^{\prime}\left[\left\{d v s^{\prime \prime} T^{\prime \prime} y^{\prime \prime}=\left\{d v s^{\prime} T^{\prime} y^{\prime}=\mathcal{E}^{\prime \prime} ; d s^{\prime} u^{\prime}\right\} ; d s^{\prime \prime} u^{\prime \prime}\right\}\right]$ and then (FIELD-ASSign-move) is applicable to

$$
\mathcal{E}_{x}\left[\mathcal{E}_{z}^{\prime}\left[\left\{d v s^{\prime \prime} T^{\prime \prime} y^{\prime \prime}=\left\{d v s^{\prime} T^{\prime} y^{\prime}=\mathcal{E}^{\prime \prime}[x . f=v] ; d s^{\prime} u^{\prime}\right\} ; d s^{\prime \prime} u^{\prime \prime}\right\}\right]\right] .
$$

Therefore, there is always a rule applicable to $\mathcal{E}[x . f=v]$.

Let $\rho=\left\{d v s T^{\prime} x=v^{\prime} ; d s v\right\}, \models_{s t} d v s$ and $\not{ }_{s t} T^{\prime} x=v^{\prime}$;. From Proposition 2 either $v \cong x$ or $v \cong r v$ for some $\models r v$. In the first case rule (Alias-ELim) is applicable. In the second, let $T^{\prime}=\mu D$ for some, $\mu$ and $D$. By cases on $\mu$.

If $\mu=$ capsule, then (CAPsule-Elim) is applicable.

If $\overline{\mu \geq \text { mut, then since } \not=s t} T^{\prime} x=r v$; we have that $r v=\left\{d v s^{\prime} v^{\prime}\right\}$. By renaming bound variables in $\left\{d v s^{\prime} v^{\prime}\right\}$ we can have that $\{d v s d s v\} \| \operatorname{dom}\left(d v s^{\prime}\right)$. Therefore, rule (Mut-Move) is applicable moving $d v s^{\prime}$ outside.

If $\mu=$ imm, let $d v s^{\prime}=d v s_{i m} d v s_{m t}$, where $d v \in d v s_{i m}$ if $\mu^{d v} \leq$ imm, and $d v \overline{\in d v s_{m t}}$ if $\mu^{d v} \geq$ mut. The side condition, $\{d v s d s v\} \| \operatorname{dom}\left(d v s_{i m}\right)$, can be satisfied by renaming of declared variables in $\left\{d v s^{\prime} v^{\prime}\right\}$. We have to show that $d v s_{i m} \| \operatorname{dom}\left(d v s_{m t}\right)$. Let $y \in \mathrm{FV}\left(r v^{\prime}\right)$ for some $\mu^{\prime} C^{\prime} x^{\prime}=r v^{\prime}$; $\in d v s_{i m}$ with $\mu^{\prime} \leq \mathrm{imm}$. So $\Gamma\left[\Gamma_{d v s_{i m}} d v s_{m t}\right] ; x s s^{\prime \prime} ; y s^{\prime \prime} \vdash r v^{\prime}: \mu^{\prime} C^{\prime}$, and from Theorem 11.1 and 3 , we have that $\Gamma\left[\Gamma_{d v s_{i m}} d v s_{m t}\right] ; x s s^{\prime \prime} ; y s^{\prime \prime} \vdash y: \mathrm{imm}_{\text {. }}$. Therefore, $y \notin \operatorname{dom}\left(d v s_{m t}\right)$, and rule (Імм-Mоve) can be applied since $d v s_{i m} \| \operatorname{dom}\left(d v s_{m t}\right)$ holds.

\subsection{Properties of expressions having immutable and capsule modifiers}

In addition to the standard soundness property, we prove two theorems stating that the capsule and imm qualifier, respectively, have the expected behaviour. A nice consequence of our non standard operational model is that this can be formally expressed and proved in a simple way.

In the two theorems, we need to trace the reduction of the right-hand side of a reference declaration. To lighten the notation, we assume in the following that expressions contain at most one declaration for a variable (no shadowing, as can be always obtained by alpha-conversion).

We need some notations and lemmas. We define contexts $\mathcal{C}_{\mu x}$ :

$$
\begin{aligned}
\mathcal{C}_{\mu x}::= & \left\{d s \mu C x=[] ; d s^{\prime} v\right\}\left|\mathcal{C}_{\mu x} . f\right| \mathcal{C}_{\mu x} \cdot m(v s) \mid v \cdot m\left(v s \mathcal{C}_{\mu x} v s^{\prime}\right) \\
& \left|\mathcal{C}_{\mu x} \cdot f=v\right| v \cdot f=\mathcal{C}_{\mu x} \mid \text { new } C\left(v s \mathcal{C}_{\mu x} v s^{\prime}\right) \\
& \left|\left\{d s T y=\mathcal{C}_{\mu x} ; d s^{\prime} v\right\}\right|\left\{d s \mathcal{C}_{\mu x}\right\}
\end{aligned}
$$

That is, in $\mathcal{C}_{\mu x}[e]$ the expression $e$ occurs as right-hand side of the (unique) declaration for reference $x$, which has qualifier $\mu$. We will simply write $\mathcal{C}$ when the $\mu x$ suffix is not relevant. 
The type assignment extracted from a context $\mathcal{C}$, denoted $\Gamma_{\mathcal{C}}$, is defined as for the general contexts.

The declaration for a variable $y$ in a context $\mathcal{C}$, denoted $\operatorname{dec}(\mathcal{C}, y)$, can be defined analogously.

The following lemma states that the type of a reference under the type assignment extracted from the surrounding context is a subtype of the declared type when the type modifier is not equal to lent. If the type modifier of the declaration is lent, the variable could be accessed in a sub-context in which, due to the (T-SWAP) rule, the variable belongs to the current mutable group and so we derive a type with the mut modifier.

Lemma 18. If $\Gamma_{\mathcal{C}} ; x s s ; y s \vdash y: \mu_{-}$, then $\operatorname{dec}(\mathcal{C}, y)=\mu^{\prime}{ }_{-} y=_{-}$; is such that $\mu^{\prime} \neq$ lent implies $\mu^{\prime} \leq \mu$.

Proof By induction on $\mathcal{C}$.

The following lemma states that a subexpression which occurs as right-handside of a declaration in a well-typed expression is well-typed, under the type assignment extracted from the surrounding context, and has a subtype of the reference type.

Lemma 19. If $\vdash \mathcal{C}_{\mu x}[e]$, then $\Gamma_{\mathcal{C}_{\mu x}} ; x s s ; y s \vdash e: \mu^{\prime}-$ for some $x s s$, ys, and $\mu^{\prime} \leq \mu$.

Proof By induction on $\mathcal{C}_{\mu x}$.

The expected behaviour of the capsule qualifier is, informally, that the reachable object subgraph denoted by a capsule reference should not contain nodes reachable from the outside, unless they are immutable. In our calculus, a reachable object subgraph is a right-value $r v$, nodes reachable from the outside are free variables, hence the condition can be formally expressed by requiring that free variables in $r v$ are declared imm or capsule in the surrounding context:

$$
\begin{aligned}
& \mathcal{C} \models \operatorname{imm-closed}(r v) \text { iff for all } y \in \operatorname{FV}(r v), \operatorname{dec}(\mathcal{C}, y)=\mu_{-} y=_{-} ; \text {with } \\
& \mu \leq \mathrm{imm}
\end{aligned}
$$

Moreover, the reachable object subgraph denoted by a capsule reference should be typable mut, since it can be assigned to a mutable reference. Altogether, the fact that the capsule qualifier guarantees the expected behaviour can be formally stated as in the theorem below, where the qualifier capsule is abbreviated c.

Theorem 20 (Capsule). If $\vdash \mathcal{C}_{\mathrm{c} x}[e]$ and $\mathcal{C}_{\mathrm{c} x}[e] \longrightarrow{ }^{\star} \mathcal{C}_{\mathrm{c} x}^{\prime}[r v]$, then:

- $\operatorname{type}(r v)=$ mut_

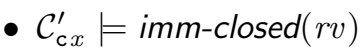


Proof By subject reduction (Theorem 15) we get $\vdash \mathcal{C}_{\mathrm{c} x}^{\prime}[r v]$. Then, from Lemma 19, $\Gamma$; xss; ys $\vdash r v$ : capsule ${ }_{\text {- with }} \Gamma=\Gamma_{\mathcal{C}_{c x}^{\prime}}$. Hence, from Theorem 11, for all $y \in \mathrm{FV}(r v), \Gamma ; x s s ; y s \vdash y: \mathrm{imm}_{-}$, and type $(r v)=$ mut . Hence, by Lemma $18, \operatorname{dec}\left(\mathcal{C}_{\mathrm{c} x}^{\prime}, y\right)=\mu_{-} y=_{-}$; with $\mu \leq \mathrm{imm}$.

Note that the context can change since it is not an evaluation context and, moreover, reduction can modify the store. Consider for instance $\mathcal{C}_{\mathrm{c} x}[e]$ to be the following expression:

mut $\mathrm{C} y=$ new $\mathrm{C}(0)$; mut $\mathrm{C} z=$ new $\mathrm{C}(\mathrm{y} . \mathrm{f}=1) ;$ capsule $\mathrm{C} \mathrm{x}=e ; \ldots$

Before reducing to a right value the initialization expression of $\mathrm{x}$, the initialization expression of $\mathbf{z}$ should be reduced, and this has a side-effect on the right value of y. Hence $\mathcal{C}_{\mathrm{c} x}^{\prime}[r v]$ is:

mut $\mathrm{C} y=$ new $\mathrm{C}(1)$; mut $\mathrm{C} z=$ new $\mathrm{C}(1) ;$ capsule $\mathrm{C} \mathrm{x}=r v ; \ldots$

The expected behaviour of the imm qualifier is, informally, that the reachable object subgraph denoted by an imm reference should not be modified through any alias. Hence, the right value of an immutable reference:

- should not be modified

- should only refer to external references which are immutable

as formally stated in the theorem below, where the qualifier imm is abbreviated i.

Theorem 21 (Immutable). If $\vdash \mathcal{C}_{\mathbf{i} x}[e]$ and $\mathcal{C}_{\mathrm{i} x}[e] \longrightarrow^{\star} \mathcal{C}_{\mathbf{i} x}^{\prime}[r v]$, then:

- $\mathcal{C}_{\mathbf{i} x}^{\prime}[r v] \longrightarrow{ }^{\star} \mathcal{C}_{\mathbf{i} x}^{\prime \prime}\left[r v^{\prime}\right]$ implies $r v=r v^{\prime}$

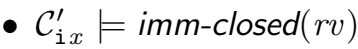

Proof The first property is directly ensured by reduction rules, since rule (FIELDASSIGN) is only applicable on $\geq$ mut references (note that the progress theorem guarantees that reduction cannot be stuck for this reason). The second property can be proved analogously to Theorem 20 above.

Again, the context can change during reduction. Consider for instance $\mathcal{C}_{\mathrm{i} x}[e]$ to be the following expression:

mut $\mathrm{C} y=$ new $\mathrm{C}(0) ;$ imm $\mathrm{C} x=e$; mut $\mathrm{C} z=$ new $\mathrm{C}(\mathrm{y} \cdot \mathrm{f}=1) ; \ldots$

In this case, the initialization expression of $x$ is firstly reduced to a right value, hence $\mathcal{C}_{\dot{1} x}^{\prime}[r v] \equiv \mathcal{C}_{\mathbf{i} x}[r v]$ is:

mut $\mathrm{C} y=$ new $\mathrm{C}(0)$; imm $\mathrm{C} \mathrm{x}=r v$; mut $\mathrm{C} z=$ new $\mathrm{C}(\mathrm{y} \cdot \mathrm{f}=1) ; \ldots$

In the following reduction steps, the context can change, for instance $\mathcal{C}_{\mathbf{i} x}^{\prime \prime}[r v]$ can be:

mut $\mathrm{C} y=$ new $\mathrm{C}(1)$; imm $\mathrm{C} \mathrm{x}=r v$; mut $\mathrm{C} z=$ new $\mathrm{C}(1) ; \ldots$

However, the right-value $r v$ cannot be modified. 


\section{Related work}

Recovery. The works most closely most closely related to ours are those based on the recovery notion, that is, the type system of Gordon et al. [15] and the Pony language [11. Indeed, the capsule property has many variants in the literature, such as isolated [15, uniqueness [6] and external uniqueness [10, balloon [1, 27, island 12. However, before the work of Gordon et al. 15, the capsule property was only ensured in simple situations, such as using a primitive deep clone operator, or composing subexpressions with the same property.

The important novelty of the type system of Gordon et al. [15] has been recovery, that is, the ability to ensure properties (e.g., capsule or immutability) by keeping into account not only the expression itself but the way the surrounding context is used. Notably, an expression which does not use external mutable references is recognized to be a capsule. In the Pony language [11] the ideas of Gordon et al. [15] are extended to a richer set of reference immutability permissions. In their terminology value is immutable, ref is mutable, box is similar to readonly as often found in literature, different from our read since it can be aliased. An ephemeral isolated reference iso` is similar to a capsule reference in our calculus, whereas non ephemeral iso references offer destructive reads and are more similar to isolated fields [15. Finally, tag only allows object identity checks and trn (transition) is a subtype of box that can be converted to value, providing a way to create values without using isolated references. The last two qualifiers have no equivalent in our work or in [15.

Our type system greatly enhances the recovery mechanism used in such previous work [15, 11] by using lent references, and rules (T-SWAP) and (T-UNRST). For instance, the examples in Figure 7 and Figure 8 would be ill-typed in [15.

A minor difference with the type systems of Gordon et al. [15] and Pony [15. 11] is that we only allow fields to be mut or imm. Allowing readonly fields means holding a reference that is useful for observing but non making remote modifications. However, our type system supports the read modifier rather than the readonly, and the read qualifier includes the lent restriction. Since something which is lent cannot be saved as part of a mut object, lent fields are not compatible with the current design where objects are born mut. The motivation for supporting read rather than readonly is discussed in a specific point later. Allowing capsule fields means that programs can store an externally unique object graph into the heap and decide later whether to unpack permanently or freeze the reachable objects. This can be useful, but, as for read versus readonly, our opinion is that this power is hard to use for good, since it requires destructive reads, as discussed in a specific point later. In most cases, the same expressive power can be achieved by having the field as mut and recovering the capsule property for the outer object.

Capabilities. In other proposals [16, 8, types are compositions of one or more capabilities. The modes of the capabilities in a type control how resources of that type can be aliased. The compositional aspect of capabilities is an important difference from type qualifiers, as accessing different parts of an object 
through different capabilities in the same type gives different properties. By using capabilities it is possible to obtain an expressivity which looks similar to our type system, even though with different sharing notions and syntactic constructs. For instance, the full encapsulation notion in $\left.{ }^{16}\right]^{13}$, apart from the fact that sharing of immutable objects is not allowed, is equivalent to the guarantee of our capsule qualifier, while our lent and their etransient achieve similar results in different ways. Their model has a higher syntactic/logic overhead to explicitly track regions. As for all work before [15], objects need to be born @unique and the type system permits to manipulate data preserving their uniqueness. With recovery [15], instead, we can forget about uniqueness, use normal code designed to work on conventional shared data, and then recover the aliasing encapsulation property.

Destructive reads. Uniqueness can be enforced by destructive reads, i.e., assigning a copy of the unique reference to a variable an destroying the original reference, see [15, 6]. Traditionally, borrowing/fractional permissions 21] are related to uniqueness in the opposite way: a unique reference can be borrowed, it is possible to track when all borrowed aliases are buried [4, and then uniqueness can be recovered. These techniques offers a sophisticate alternative to destructive reads. We also wish to avoid destructive reads. In our work, we ensure uniqueness by linearity, that is, by allowing at most one use of a capsule reference.

In our opinion, programming with destructive reads is involved and hurts the correctness of the program, since it leads to the style of programming outlined below, where a.f is a unique/isolated field with destructive read.

a.f $=\mathrm{c}$.doStuff(a.f)//style suggested by destructive reads

The object referenced by a has an unique/isolated field $f$ containing an object $\mathrm{b}$. This object $\mathrm{b}$ is passed to a client $\mathrm{c}$, which can use (potentially modifying) it. A typical pattern is that the result of such computation is a reference to $\mathrm{b}$, which a can then recover. This approach allows isolated fields, as shown above, but has a serious drawback: an isolated field can become unexpectedly not available (in the example, during execution of doStuff), hence any object contract involving such field can be broken. This can cause subtle bugs if a is in the reachable object graph of $\mathrm{c}$.

In our approach, the capsule qualifier cannot be applied to fields. Indeed, the "only once" use of capsule variables makes no sense on fields. However, we support the same level of control of the reachable object graph by passing mutable objects to clients as lent, in order to control aliasing behaviour. That is, the previous code can be rewritten as follows:

c.doStuff(a.f())//our suggested style

\footnotetext{
${ }^{13}$ This paper includes a very good survey of work in this area, notably explaining the difference between external uniqueness [10] and full encapsulation.
} 
where a.f () is a getter returning the field as lent. Note how, during the execution of doStuff, a.f() is still available, and, after the execution of doStuff, the aliasing relation for this field is the same as it was before doStuff was called.

Ownership. A closely related stream of research is that on ownership (see an overview in [9]) which, however, offers an opposite approach. In the ownership approach, it is provided a way to express and prove the ownership invariant ${ }^{14}$ which, however, is expected to be guaranteed by defensive cloning, as explained below. In our approach, instead, the capsule concept models an efficient ownership transfer. In other words, when an object $x$ is "owned" by another object $y$, it remains always true that $y$ can be only accessed only through $x$, whereas the capsule notion is more dynamic: a capsule can be "opened", that is, assigned to a standard reference and modified, and then we can recover the original capsule guarantee.

For example, assuming a graph with a list of nodes, and a constructor taking in input such list, the following code establishes the ownership invariant using capsule, and ensures that it cannot be violated using lent.

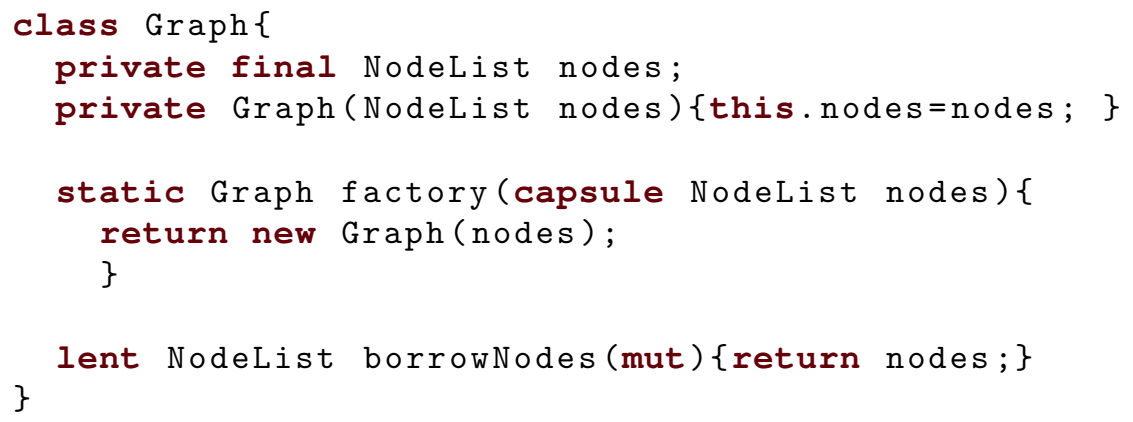

Requiring the parameter of the factory method to be a capsule guarantees that the list of nodes provided as argument is not referred from the external environment. The factory moves an isolated portion of store as local store of the newly created object. Cloning, if needed, becomes responsibility of the client which provides the list of nodes to the graph. The getter tailors the exposure level of the private store.

Without aliasing control (capsule qualifier), in order to ensure ownership of its list of nodes, the factory method should clone the argument, since it comes from an external client environment. This solution, called defensive cloning [3], is very popular in the Java community, but inefficient, since it requires to duplicate the reachable object graph of the parameter, until immutable nodes are

\footnotetext{
${ }^{14}$ Ownership invariant (owner-as-dominator): Object $o_{1}$ is owned by object $o_{2}$ if in the object graph $O_{2}$ is a dominator node for $o_{1}$; that is, all paths from the roots of the graph (the stack variables) to $o_{1}$ pass throw $\mathrm{O}_{2}$. Ownership invariant (owner-as-modifier): Object $o_{1}$ is owned by object $o_{2}$ if any field update over $o_{1}$ is initiated by $o_{2}$, that is, a call of a method of $\mathrm{O}_{2}$ is present in the stack trace.
} 
reached 15 Indeed, many programmers prefer to write unsafe code instead of using defensive cloning for efficiency reasons. However, this unsafe approach is only possible when programmers have control of the client code, that is, they are not working in a library setting. Indeed many important Java libraries (including the standard Java libraries) today use defensive cloning to ensure ownership of their internal state.

As mentioned above, our approach is the opposite of the one offered by many ownership approaches, which provide a formal way to express and prove the ownership invariant that, however, are expected to be guaranteed by defensive cloning. We, instead, model an efficient ownership transfer through the capsule concept, then, duplication of memory, if needed, is performed on the client $\operatorname{sid} 16$.

Moreover, depending on how we expose the owned data, we can closely model both owners-as-dominators (by providing no getter) and owners-as-qualifiers (by providing a read getter). In the example, the method borrowNodes is an example of a lent getter, a third variant besides the two described on page 21. This variant is particularly useful in the case of a field which is owned, indeed, Graph instances can release the mutation control of their nodes without permanently losing the aliasing control.

In our approach all properties are deep. On the opposite side, most ownership approaches allows one to distinguish subparts of the reachable object graph that are referred but not logically owned. This viewpoint has many advantages, for example the Rust languag $\Phi^{17}$ leverages on ownership to control object deallocation without a garbage collector. Rust employs a form of uniqueness that can be seen as a restricted "owners-as-dominators" discipline. Rust lifetime parameters behave like additional ownership parameters [22].

However, in most ownership based approaches it is not trivial to encode the concept of full encapsulation, while supporting (open) sub-typing and avoiding defensive cloning. This depends on how any specific ownership approach entangles subtyping with gaining extra ownership parameters and extra references to global ownership domains.

Readable notion. Our read qualifier is different from readonly as used, e.g., in [2]. An object cannot be modified through a readable/readonly reference. However, read also prevents aliasing. As discussed in [5], readonly semantics can be easily misunderstood by programmers. Indeed, some wrongly believe it means immutable, whereas the object denoted by a readonly reference can be modified through other references, while others do not realize that readonly

\footnotetext{
${ }^{15}$ In most languages, for owner-as-modifier defensive cloning is needed only when new data is saved inside of an object, while for owner-as-dominator it is needed also when internal data are exposed.

16 Other work in literature supports ownership transfer, see for example [20, 10. In literature it is however applied to uniquess/external uniqueness, thus not the whole reachable object graph is transfered.

${ }^{17}$ rust-lang.org
} 
data can still be saved in fields, and thus used as a secondary window to observe the change in the object state. Our proposal addresses both problems, since we explicitly support the imm qualifier, hence it is more difficult for programmers to confuse the two concepts, and our read (readonly + lent) data cannot be saved in client's fields.

Javari 29] also supports the readonly type qualifier, and makes a huge design effort to support assignable and mutable fields, to have fine-grained readonly constraints. The need of such flexibility is motivated by performance reasons. In our design philosophy, we do not offer any way of breaking the invariants enforced by the type system. Since our invariants are very strong, we expect compilers to be able to perform optimization, thus recovering most of the efficiency lost to properly use immutable and readable objects.

\section{Conclusion}

The key contributions of the paper are:

- A powerful type system for control of mutation and aliasing in class-based imperative languages, providing: type qualifiers for restricting the use of references; rules for recovering a less restrictive type at the price of constraining the use of other references; rules for temporarily unconstrain such references for typing subexpressions.

- A non standard operational model of the language, relying on the language's ability to represent store.

We have extensively illustrated the former feature in Section 4, here we briefly discuss the latter.

In our operational model, aliasing properties are directly expressed at the syntactic level. Notably, in a subterm $e$ of a program, objects reachable from other parts of the program are simply those denoted by free variables in $e$, whereas local objects are those denoted by local variables declared in $e$. In other terms, the portion of memory only reachable from e is encoded in e itself. For instance, in

mut $D$ y=new $D(0)$;

capsule $\mathrm{C} z=\{$ mut $\mathrm{D} x=$ new $\mathrm{D}(1) ;$ new $\mathrm{C}(\mathrm{x}, \mathrm{x})\}$

it is immediately clear that the reference $z$ denotes an isolated portion of memory, since its right-value is a closed expression. In the conventional model, instead, memory is flat. For instance, the previous example would be modeled having three locations, say $\iota_{y}, \iota_{z}$, and $\iota_{x}$, all at the top-level, hence the fact that $\iota_{x}$ is not reachable from $\iota_{y}$ should be reconstructed inspecting the store.

In our opinion, this offers a very intuitive and simple understanding of when a subterm of a program can be safely typed capsule, as we have exploited in the examples in Section 3. More generally, we argue that our model is more adequate for didactic purposes since it does not rely on run-time structures that do not exist in the source program, see [25] for an extended discussion on this. 
Another advantage is that, being the store encoded in terms, proofs can be done by structural induction, whereas in the traditional model invariants on the memory should be proved showing that all locations satisfy a given property. This is especially important when using a proof assistant, such as Coq, which we plan as further work.

On the opposite side, a disadvantage of our model is the fact that it is possibly more esoteric for people used to the other one. Moreover, since isolation is encoded by scoping, some care is needed to avoid scope extrusion during reduction. Notably, a field access on a reference which points to an isolated portion of store (a block) cannot produce just a reference.

As mentioned in Section 3 , the type system presented in this paper, as those in previous work on recovery [15, 11, uses rules which are not syntax-directed, hence transparent to the programmer, in the sense that they are applied when needed. The programmer can simply rely on the fact that expressions are capsule or imm, respectively, in situations where this is intuitively expected. Of course, the negative counterpart is that the type system is not algorithmic. This is due, as said above, to the presence of non structural rules, which are, besides standard subsumption, recovery rules (T-CAPSUlE) and (T-IMm), rules (T-SWAP) and (T-UNRST) for swapping and unrestricting, and of the rule (т-вLоск) for blocks, where variables declared lent are assigned to groups in an arbitrary way.

We considered two different ways to provide an algorithmic version of the type system.

The first way is to modify the type system as it is usually done for the subsumption rule, so that non structural rules are applied, roughly, only when needed. We did not formally develop this solution, but the technique is applied in the prototype of L42, a novel programming language designed to support massive use of libraries ${ }^{18}$ The current L42 prototype is important as proof-ofevidence that the type system presented in this paper not only can be implemented, but also smoothly integrated with other features of a realistic language. For testing purposes a small step reduction closely resembling the one presented in this paper is also implemented.

The other way is a different design of the type system based on type inference. The basic idea is to compute the sharing relations possibly introduced by the evaluation of an expression. A preliminary version of this approach is in [14.

A first, informal, presentation of the type modifiers in this paper has been given in 27]. In 28] we have provided a formal type system including the capsule and lent modifiers, and a preliminary version of capsule recovery. In [13. we have added the immutable and readable modifiers, and the immutability recovery. This paper largely extends [13. The novel contributions include reduction rules, more examples, theorems about the behaviour of modifiers, and proofs of results. Imperative calculi where the block construct models store

\footnotetext{
${ }^{18}$ Description and prototype for the full language (in progress) can be found at L42.is. For testing purposes a small step reduction closely resembling the one presented in this paper is also implemented.
} 
have been introduced in [25, 77, and used in [28, 13.

Concerning further work, it would be interesting to investigate how to extend mainstream languages, such as, e.g., Java, with our type qualifiers, and to develop implementations of the calculus presented in this paper, possibly using, e.g., Coq, to also be able to formalize and prove properties.

On the more theoretical side, the first direction we plan to explore is the relation between the non-algorithmic type system presented in this paper and the previously mentioned type system based on inference of sharing relations [14. We also plan to formally state and prove behavioural equivalence of the calculus with the conventional imperative model.

The fact that our type system tracks requirements on the type context makes it a form of coeffect type system in the sense of 23 . We plan to investigate better the relation. We believe that the novel operational model presented in this paper has the potential of achieving a better understanding of aliasing.

As a long term goal, we also plan to investigate (a form of) Hoare logic on top of our model. We believe that the hierarchical structure of our memory representation should help local reasoning, allowing specifications and proofs to mention only the relevant portion of the memory, analogously to what is achieved by separation logic [24. Finally, it should be possible to use our approach to enforce safe parallelism, on the lines of [15, 27].

Acknowledgement. We are indebted to the anonymous TCS referees for the thorough job they did reviewing our paper and for their valuable suggestions which improved it greatly. We also thank the ICTCS'16 referees for their helpful comments on the preliminary version of the paper.

\section{Bibliography}

\section{References}

[1] P. S. Almeida, Balloon types: Controlling sharing of state in data types, in: ECOOP'97 - Object-Oriented Programming, vol. 1241 of Lecture Notes in Computer Science, Springer, 1997.

[2] A. Birka, M. D. Ernst, A practical type system and language for reference immutability, in: ACM SIGPLAN Conference on Object-Oriented Programming, Systems, Languages and Applications (OOPSLA 2004), 2004.

[3] J. Bloch, Effective Java (2Nd Edition) (The Java Series), 2nd ed., Prentice Hall PTR, 2008.

[4] J. Boyland, Alias burying: Unique variables without destructive reads, Softw. Pract. Exper. 31 (6) (2001) 533-553.

[5] J. Boyland, Why we should not add readonly to Java (yet), Journal of Object Technology 5 (5) (2006) 5-29. 
[6] J. Boyland, Semantics of fractional permissions with nesting, ACM Transactions on Programming Languages and Systems 32 (6).

[7] A. Capriccioli, M. Servetto, E. Zucca, An imperative pure calculus, Electronic Notes in Theoretical Computer Science 322 (2016) 87-102.

[8] E. Castegren, T. Wrigstad, Reference capabilities for concurrency control, in: S. Krishnamurthi, B. S. Lerner (eds.), ECOOP'16 - Object-Oriented Programming, vol. 56 of LIPIcs, Schloss Dagstuhl - Leibniz-Zentrum fuer Informatik, 2016.

[9] D. Clarke, J. Östlund, I. Sergey, T. Wrigstad, Ownership types: A survey, in: D. Clarke, J. Noble, T. Wrigstad (eds.), Aliasing in Object-Oriented Programming. Types, Analysis and Verification, vol. 7850 of Lecture Notes in Computer Science, Springer, 2013, pp. 15-58.

[10] D. Clarke, T. Wrigstad, External uniqueness is unique enough, in: ECOOP'03 - Object-Oriented Programming, vol. 2473 of Lecture Notes in Computer Science, Springer, 2003.

[11] S. Clebsch, S. Drossopoulou, S. Blessing, A. McNeil, Deny capabilities for safe, fast actors, in: E. G. Boix, P. Haller, A. Ricci, C. Varela (eds.), International Workshop on Programming Based on Actors, Agents, and Decentralized Control, AGERE! 2015, ACM Press, 2015.

[12] W. Dietl, S. Drossopoulou, P. Müller, Generic universe types, in: ECOOP'07 - Object-Oriented Programming, vol. 4609 of Lecture Notes in Computer Science, Springer, 2007.

[13] P. Giannini, M. Servetto, E. Zucca, Types for immutability and aliasing control, in: ICTCS'16 - Italian Conf. on Theoretical Computer Science, vol. 1720 of CEUR Workshop Proceedings, CEUR-WS.org, 2016. URL http://ceur-ws.org/Vol-1720/full5.pdf

[14] P. Giannini, M. Servetto, E. Zucca, A type and effect system for sharing, in: OOPS'17 - Object-Oriented Programming Languages and Systems, Track at SAC'17 - ACM Symp. on Applied Computing, 2017, to appear.

[15] C. S. Gordon, M. J. Parkinson, J. Parsons, A. Bromfield, J. Duffy, Uniqueness and reference immutability for safe parallelism, in: ACM SIGPLAN Conference on Object-Oriented Programming, Systems, Languages and Applications (OOPSLA 2012), ACM Press, 2012.

[16] P. Haller, M. Odersky, Capabilities for uniqueness and borrowing, in: T. D'Hondt (ed.), ECOOP'10 - Object-Oriented Programming, vol. 6183 of Lecture Notes in Computer Science, Springer, 2010.

[17] J. Hogg, Islands: Aliasing protection in object-oriented languages, in: A. Paepcke (ed.), ACM Symp. on Object-Oriented Programming: Systems, Languages and Applications 1991, ACM Press, 1991. 
[18] A. Igarashi, B. C. Pierce, P. Wadler, Featherweight Java: a minimal core calculus for Java and GJ, ACM Transactions on Programming Languages and Systems 23 (3) (2001) 396-450.

[19] R. L. B. Jr., V. S. Adve, D. Dig, S. V. Adve, S. Heumann, R. Komuravelli, J. Overbey, P. Simmons, H. Sung, M. Vakilian, A type and effect system for deterministic parallel java, in: S. Arora, G. T. Leavens (eds.), ACM SIGPLAN Conference on Object-Oriented Programming,Systems, Languages and Applications (OOPSLA 2009), ACM Press, 2009.

[20] P. Müller, A. Rudich, Ownership transfer in universe types, in: R. P. Gabriel, D. F. Bacon, C. V. Lopes, G. L. S. Jr. (eds.), ACM SIGPLAN Conference on Object-Oriented Programming, Systems, Languages and Applications (OOPSLA 2007), ACM Press, 2007.

[21] K. Naden, R. Bocchino, J. Aldrich, K. Bierhoff, A type system for borrowing permissions, in: ACM Symp. on Principles of Programming Languages 2012, ACM Press, 2012.

[22] J. Östlund, T. Wrigstad, D. Clarke, B. Åkerblom, Ownership, uniqueness, and immutability, in: R. F. Paige, B. Meyer (eds.), International Conference on Objects, Components, Models and Patterns, vol. 11 of Lecture Notes in Computer Science, Springer, 2008.

[23] T. Petricek, D. A. Orchard, A. Mycroft, Coeffects: a calculus of contextdependent computation, in: J. Jeuring, M. M. T. Chakravarty (eds.), Intl. Conf. on Functional Programming 2014, ACM Press, 2014.

[24] J. C. Reynolds, Separation logic: A logic for shared mutable data structures, in: Proc. IEEE Symp. on Logic in Computer Science 2002, IEEE Computer Society, 2002.

[25] M. Servetto, L. Groves, True small-step reduction for imperative objectoriented languages, in: W. Dietl (ed.), FTfJP'13- Formal Techniques for Java-like Programs, ACM Press, 2013.

[26] M. Servetto, J. Mackay, A. Potanin, J. Noble, The billion-dollar fix - safe modular circular initialisation with placeholders and placeholder types, in: ECOOP'13 - Object-Oriented Programming, vol. 7920 of Lecture Notes in Computer Science, Springer, 2013.

[27] M. Servetto, D. J. Pearce, L. Groves, A. Potanin, Balloon types for safe parallelisation over arbitrary object graphs, in: WODET 2014 - Workshop on Determinism and Correctness in Parallel Programming, 2013.

[28] M. Servetto, E. Zucca, Aliasing control in an imperative pure calculus, in: X. Feng, S. Park (eds.), Programming Languages and Systems - 13th Asian Symposium (APLAS), vol. 9458 of Lecture Notes in Computer Science, Springer, 2015. 
[29] M. S. Tschantz, M. D. Ernst, Javari: Adding reference immutability to Java, in: ACM SIGPLAN Conference on Object-Oriented Programming, Systems, Languages and Applications (OOPSLA 2005), ACM Press, 2005.

[30] Y. Zibin, A. Potanin, P. Li, M. Ali, M. D. Ernst, Ownership and immutability in generic Java, in: ACM SIGPLAN Conference on ObjectOriented Programming,Systems, Languages and Applications (OOPSLA 2010), 2010.

\section{Appendix A. Auxiliary definitions}

$\operatorname{HB}(\mathcal{E})$ :

$$
\mathrm{HB}([])=\emptyset
$$

$\mathrm{HB}(\{d v s \quad T x=\mathcal{E} ; d s e\})=\mathrm{HB}(\mathcal{E}) \cup \operatorname{dom}(d v s) \cup \operatorname{dom}(d s) \cup\{x\}$

$\mathrm{FV}(e)$ :

$\mathrm{FV}(x)=\{x\}$

$\mathrm{FV}(e . f)=\mathrm{FV}(e)$

$\mathrm{FV}\left(e_{0} \cdot m\left(e_{1}, \ldots, e_{n}\right)\right)=\mathrm{FV}\left(e_{0}\right) \cup \ldots \cup \mathrm{FV}\left(e_{n}\right)$

$\mathrm{FV}\left(e . f=e^{\prime}\right)=\mathrm{FV}(e) \cup \mathrm{FV}\left(e^{\prime}\right)$

$\mathrm{FV}\left(\right.$ new $\left.C\left(e_{1}, \ldots, e_{n}\right)\right)=\mathrm{FV}\left(e_{1}\right) \cup \ldots \cup \mathrm{FV}\left(e_{n}\right)$

$\mathrm{FV}(\{d s e\})=\mathrm{FV}(d s) \cup(\mathrm{FV}(e) \backslash \operatorname{dom}(d s))$

$\mathrm{FV}\left(T_{1} x_{1}=e_{1} ; \ldots T_{n} x_{n}=e_{n} ;\right)=\left(\mathrm{FV}\left(e_{1}\right) \cup \ldots \cup \mathrm{FV}\left(e_{n}\right)\right) \backslash\left\{x_{1}, \ldots, x_{n}\right\}$

$e[v / x]$ :

$x[v / x]=e$

$z[v / x]=z$ if $z \neq x$

e.f $[v / x]=e[v / x] . f$

$e_{0} \cdot m\left(e_{1}, \ldots, e_{n}\right)[v / x]=e_{0}[v / x] \cdot m\left(e_{1}[v / x], \ldots, e_{n}[v / x]\right)$

$\left(e . f=e^{\prime}\right)[v / x]=e[v / x] . f=e^{\prime}[v / x]$

new $C\left(e_{1}, \ldots, e_{n}\right)[v / x]=$ new $C\left(e_{1}[v / x], \ldots, e_{n}[v / x]\right)$

$\{d s e\}[v / x]=\{d s[v / x] e[v / x]\}$ if $x \notin \operatorname{dom}(d s)$

$\{d s e\}[v / x]=\{d s e\}$ if $x \in \operatorname{dom}(d s)$

$\left(T_{1} x_{1}=e_{1} ; \ldots T_{n} x_{n}=e_{n} ;\right)[v / x]=T_{1} x_{1}=e_{1}[v / x] ; \ldots T_{n} x_{n}=e_{n}[v / x]$;

\section{Appendix B. Proofs omitted from Section 6}

Proof of Lemma 3 . By induction on type derivations.

If the last rule in $\mathcal{D}$ is a structural rule, then $\mathcal{D}^{\prime}=\mathcal{D}$.

If the last rule in $\mathcal{D}$ is (T-suB), by induction hypothesis on the sub-derivation of the premise of (T-suB) and transitivity of $\leq$ we derive the result.

Let $\mathcal{D}$ end with an application of (T-Capsule) of (T-Imm), then

$$
\mathcal{D}_{p}: \Gamma ; x s s\left(\operatorname{dom}^{\text {mut }}(\Gamma) \backslash x s s\right)[\emptyset] ; y s^{\prime \prime} \vdash e: \mu^{\prime} C
$$

where $y s^{\prime \prime}=y s$ or $y s^{\prime \prime}=\operatorname{dom}^{\geq \operatorname{mut}}(\Gamma)$, is the sub-derivation of the premise of the last rule. Note that the mutable group is always empty. By induction hypothesis on $\mathcal{D}_{p}$, there is a sub-derivation of $\mathcal{D}^{\prime}$ of $\mathcal{D}_{p}$ such that $\mathcal{D}^{\prime}: \Gamma ; x s s^{\prime}\left[x s^{\prime}\right] ; y s^{\prime} \vdash e$ : $\mu^{\prime \prime} C$ ends with the application of a structural rule, and $x s s\left(\operatorname{dom}^{\text {mut }}(\Gamma) \backslash x s s\right)=$ 
$x s s^{\prime} x s^{\prime}$. This proves the result.

If the last rule applied is (T-UnRST) $\Gamma ; x s s[x s] ; \emptyset \vdash e: \mu C$, by induction hypothesis on the sub-derivation of the premise of the rule, we derive the result.

If the last rule applied is (T-SwAP), let $x s s=x s s_{1} x s_{1}$

$$
\mathcal{D}_{p}: \Gamma ; x s s_{1} x s\left[x s_{1}\right] ; y s \vdash e: \mu^{\prime} C
$$

By induction hypothesis on $\mathcal{D}_{p}$ there is a sub-derivation $\mathcal{D}^{\prime}$ such that $\mathcal{D}^{\prime}$ : $\Gamma ; x s s^{\prime}\left[x s^{\prime}\right] ; y s^{\prime} \vdash e: \mu^{\prime \prime} C$ ends with the application of a structural rule, $x s s_{1} x s x s_{1}=$ $x s s^{\prime} x s^{\prime}$, and either $\mu^{\prime}=$ capsule or $\mu^{\prime}=$ imm or $\mu^{\prime \prime} \leq \mu^{\prime}$. Since $x s s=x s s_{1} x s_{1}$ we have that $x s s x s=x s s^{\prime} x s^{\prime}$, moreover, since $\mu^{\prime} \leq \mu$ by transitivity of $\leq$ we have the result.

Proof of Lemma 5. By induction on the derivation $\Gamma$; $x s s ; y s \vdash x: T$. Consider the last rule applied in the derivation.

If the last rule is (T-VAR), then either $T=\Gamma(x)$ or $T=\operatorname{lent} C$ if $\Gamma(x)=$ mut $C$ and $x \in x s s$. Therefore, in both cases $\Gamma(x) \leq T$. Otherwise the last rule applied must be a non-structural rule.

If the last rule applied is (T-SuB), the result follows by induction hypothesis, and transitivity of $\leq$.

If the last rule applied is (T-swap), then $\Gamma ; x s s^{\prime} ; y s \vdash x: T^{\prime}$, where $T^{\prime} \leq T$. Therefore, again by induction induction hypothesis, and transitivity of $\leq$ we get the result.

If the last rule applied is (T-UNRST) the result follows by induction hypothesis.

If the last rule applied is (T-CApsule), then $\Gamma$; xss $x s ; y s \vdash x$ : mut $C$ where $x s=\operatorname{dom}^{\text {mut }}(\Gamma) \backslash x s s$, and $T=$ capsule $C$. By induction hypothesis, $\Gamma(x) \leq$ mut $C$. So, either $\Gamma(x)=$ capsule $C$ or $\Gamma(x)=\operatorname{mut} C$. In the first case $\Gamma(x)=T$. The second case is not possible, since $x \in$ xss $x s$, and therefore $\Gamma ; x s s \quad x s ; y s \vdash x$ : lent $C$, so rule (T-Capsule) cannot be applied.

Finally, if the last rule applied is (Т-Імм), it must be that $\Gamma$; xss $x s ; \operatorname{dom}^{\geq \operatorname{mut}}(\Gamma) \vdash$ $x: \operatorname{read} C$. So, $x \notin \operatorname{dom}^{\geq \operatorname{mut}}(\Gamma)$, and therefore $\Gamma(x)=$ capsule $C$ or $\Gamma(x)=$ imm $C$. In both cases $\Gamma(x) \leq T=\operatorname{imm} C$.

Let $\Gamma$; xss; ys $\vdash x: \mu C$ with $\mu \leq$ imm. We have that $\Gamma(x) \leq \mu C$. Therefore, if $x s s^{\prime}$ and $y s^{\prime}$ are such that $\vdash \Gamma ; x s s^{\prime} ; y s^{\prime}$, then $x \notin x s s^{\prime}$ and $x \notin y s^{\prime}$. So $\Gamma ; x s s^{\prime} ; y s^{\prime} \vdash x: \mu C$.

Proof of Lemma 7] Let $\mathcal{D}: \Gamma ; x s s[x s] ; y s \vdash\{d s v\}: \mu C$. From Lemma 3 there is a sub-derivation $\mathcal{D}^{\prime}: \Gamma ; x s s^{\prime \prime}\left[x s^{\prime \prime}\right] ; y s^{\prime \prime} \vdash\{d s v\}: \mu^{\prime} C$ of $\mathcal{D}$ which ends with an application of rule (Т-ВLоск) such that

(a) $x s s x s=x s s^{\prime \prime} x s^{\prime \prime}$ and

(b) $\mu \neq$ imm and $\mu \neq$ capsule implies $\mu^{\prime} \leq \mu$.

From rule (Т-вцоск) and Lemma 41 we have that

(c) $\Gamma\left[\Gamma_{d s}\right] ; x s s^{\prime} ; y s^{\prime} \vdash d s$ OK and $\Gamma\left[\Gamma_{d s}\right] ; x s s^{\prime} ; y s^{\prime} \vdash v: \mu^{\prime} C$ where

(d) $\left(x s s^{\prime \prime} x s^{\prime \prime}\right) \backslash \operatorname{dom}\left(\Gamma_{d s}\right) \sqsubseteq x s s^{\prime} x s^{\prime}$.

From (a) and (d) we derive that ( xss xs $) \backslash \operatorname{dom}\left(\Gamma_{d s}\right) \sqsubseteq x s s^{\prime} x s^{\prime}$ which, with (b) and (c), proves the result.

Proof of Lemma 8 
1. From Lemma 4 we have that $\Gamma$; xss $[x s]$; ys $\vdash$ new $C\left(z_{1}, \ldots, z_{n}\right):$ mut $C$ is derived applying rule (T-New). Therefore $\Gamma$; xss $[x s] ; y s \vdash z_{i}: \mu_{i} C_{i} \forall i \in$ 1..n. If $T_{i}=$ mut, then $z_{i} \notin x s s$ and therefore $z_{i} \in x s$. If $T_{i}=\mathrm{imm}$, from Lemma 5, we have $\Gamma ; x s s[x s] ; y s \vdash z_{i}: \mu C$ with $\mu \leq$ imm.

2. Let $\mathcal{D}: \Gamma ; x s s[\emptyset] ; \operatorname{dom}^{\geq \operatorname{mut}}(\Gamma) \vdash$ new $C\left(z_{1}, \ldots, z_{n}\right): \operatorname{read} C$. There is a sub-derivation $\mathcal{D}^{\prime}$ of $\mathcal{D}$ ending with an application of (T-New), which can be either the premise of rule (T-SuB) or of rule (T-Swap).

In the first case, $\Gamma$; xss $[\emptyset]$; $\operatorname{dom}^{\geq \text {mut }}(\Gamma) \vdash$ new $C(x s):$ mut $C$. Therefore, from clause 1. of this lemma, since the current mutable group is empty, we have that for all $i \in 1 . . n, \Gamma ; x s s ; y s \vdash z_{i}:$ imm $C_{i}$.

In the second case, $\Gamma ; x s s^{\prime}\left[x s^{\prime}\right]$; dom ${ }^{\text {mut }}(\Gamma) \vdash$ new $C\left(z_{1}, \ldots, z_{n}\right):$ mut $C$ for some $x s s^{\prime} x s^{\prime}=x s s$. Assume that, for some $i, 1 \leq i \leq n, T_{i}=\mu_{i} C_{i}$ with $\mu_{i} \geq$ mut, and therefore, $z_{i} \in \operatorname{dom}^{\geq \text {mut }}(\Gamma)$. Rule (T-VAR) is not be applicable to derive a type for $z_{i}$. (Rule (T-UnRst) is not applicable to unlock variables, since the variable has type $\mu_{i} C_{i}$ with $\mu_{i} \geq$ mut.) Therefore, for all $i \in 1 . . n$, we have that $\Gamma\left(z_{i}\right)=\mu C_{i}$ with $\mu \leq$ imm.

Proof of Lemma 9. Let $\Gamma$; xss $[x s] ; y s \vdash\left\{\begin{array}{lll}d v s & w\end{array}\right\}$ : mut $C$ where $w=x$ or $w=$ new $C\left({ }_{\_}\right)$. From Lemma 7

(a) $\Gamma\left[\Gamma_{d v s}\right] ; x s s^{\prime}\left[x s^{\prime}\right] ; y s^{\prime} \vdash w: \mu^{\prime} C$

(b) $\Gamma\left[\Gamma_{d v s}\right] ; x s s^{\prime}\left[x s^{\prime}\right] ; y s^{\prime} \vdash \mu_{i} C_{i} z_{i}=r v_{i}$; OK $\forall i \in 1 . . n$

(c) $(x s s x s) \backslash \operatorname{dom}\left(\Gamma_{d v s}\right) \sqsubseteq x s s^{\prime} x s^{\prime}$ and

(d) $\mu^{\prime} \leq$ mut.

We may assume that $\mu^{\prime}=$ mut, since $\mu^{\prime}=$ capsule would imply that (a) was obtained by a (T-CAPsule) rule and so $\Gamma\left[\Gamma_{d v s}\right] ; x s s^{\prime} x s^{\prime} ; y s^{\prime} \vdash w:$ mut $C$.

From the definition of well-formed right-values of Figure 11 we have that $d v s=$ $d v s_{\mid \mathrm{FV}(w)}$, that is $\forall j \in 1 . . n$ we have that $\mathrm{FV}(w) \stackrel{d v s}{\longrightarrow} z_{j}$.

We want to prove that $\forall j \in 1 . . n$, if $\mathrm{FV}(w) \stackrel{d v s}{\longrightarrow} z_{j}$ and $\Gamma\left[\Gamma_{d v s}\right]\left(z_{j}\right)=$ mut $C_{j}$, then

- $z_{j} \in x s^{\prime}$, and

- if $z \in \mathrm{FV}\left(r v_{j}\right)$ and $\Gamma\left[\Gamma_{d v s}\right](z)=$ mut $D$, then $z \in x s^{\prime}$, otherwise $\Gamma\left[\Gamma_{d v s}\right](z)=$ $\mu D$ with $\mu \leq$ imm.

By definition of connected, either $z_{j} \in \mathrm{FV}(w)$ or $z_{j} \in \mathrm{FV}\left(r v_{i}\right)$ and $\mathrm{FV}(w) \stackrel{d v s}{\longrightarrow} z_{i}$. If $z_{j} \in \mathrm{FV}(w)$ and $w=$ new $C\left({ }_{-}\right)$by (a), (d) and Lemma 8.1 we have that either $z_{j} \bar{\epsilon} s^{\prime}$ or $\Gamma\left[\Gamma_{d v s}\right]\left(z_{j}\right)=\mu D$ with $\mu \leq \mathrm{imm}$.

If $z_{j} \in \mathrm{FV}(w)$ and $w=z_{j}$ then, from (a) and (d) we get $z_{j} \in x s^{\prime}\left(z_{j} \in x s s^{\prime}\right.$ would imply $\Gamma\left[\Gamma_{d v s}\right] ; x s s^{\prime}\left[x s^{\prime}\right] ; y s^{\prime} \vdash w$ : lent $\left.C\right)$.

If $z_{j} \in \mathrm{FV}\left(r v_{i}\right)$ and $\mathrm{FV}(w) \stackrel{d v s}{\longrightarrow} z_{i}$, by inductive hypothesis on the connection relation, we have that

(1) $z_{i} \in x s^{\prime}$, and 
(2) if $z \in \mathrm{FV}\left(r v_{i}\right)$ and $\Gamma\left[\Gamma_{d v s}\right](z)=$ mut $D$, then $z \in x s^{\prime}$, otherwise $\Gamma\left[\Gamma_{d v s}\right](z)=$ $\mu D$ with $\mu \leq$ imm.

From (b) and Definition 6 we derive that $\Gamma\left[\Gamma_{d v s}\right] ; x s s\left[x s^{\prime}\right] ; y s \vdash r v_{i}:$ mut $C_{i}$. If $z_{j}$ is such that $\Gamma\left[\Gamma_{d v s}\right]\left(z_{j}\right)=$ mut $C_{j}$, from (2), $z_{j} \in x s^{\prime}$. Again, from (b) and Definition 6 we derive that $\Gamma\left[\Gamma_{d v s}\right] ; x s s\left[x s^{\prime}\right] ; y s \vdash r v_{j}$ : mut $C_{j}$. Let $z \in$ $\mathrm{FV}\left(r v_{j}\right)$. If $r v_{j}=$ new $D\left({ }_{-}\right)$then from Lemma 81 we derive the result. If $r v_{j}=\left\{d v s^{\prime} w^{\prime}\right\}$, then by inductive hypothesis on $\left\{d v s^{\prime} w^{\prime}\right\}$ we have that either $z \in x s^{\prime}$ or $\Gamma\left[\Gamma_{d v s}\right](z)=\mu D$ with $\mu \leq$ imm.

Proof of Lemma 12. By induction on $\mathcal{G}$. For $\mathcal{G}=[$ ] clause 1 derives from Lemma 3, and clause 2 is obvious.

If $\mathcal{G}=\left\{d s^{\prime} T x=\mathcal{G}^{\prime} ; d s^{\prime \prime} v\right\}$, then $\Gamma ; x s s ; y s \vdash\left\{d s^{\prime} T_{x} x=\mathcal{G}^{\prime}[e] ; d s^{\prime \prime} v\right\}: T$. Let $d s$ be $d s^{\prime} T_{x} x=\mathcal{G}^{\prime}[e] ; d s^{\prime \prime}$. From Lemma 7, we have that $\Gamma\left[\Gamma_{d s}\right] ; x s s^{\prime} ; y s^{\prime} \vdash d s$ OK where

(a) ( xss xs $) \backslash \operatorname{dom}\left(\Gamma_{d s}\right) \sqsubseteq x s s^{\prime} x s^{\prime}$.

From $\Gamma\left[\Gamma_{d s}\right] ; x s s^{\prime} ; y s^{\prime} \vdash d s$ OK we get $\Gamma\left[\Gamma_{d s}\right] ; x s s^{\prime} ; y s^{\prime} \vdash \mu_{x} C_{x} x=\mathcal{G}^{\prime}[e] ; x=\mathcal{G}^{\prime}[e]$; OK. We have two cases: either $\mu_{x} \neq$ lent and

- $\mathcal{D}_{x}: \Gamma\left[\Gamma_{d s}\right] ; x s s^{\prime}\left[x s^{\prime}\right] ; y s^{\prime} \vdash \mathcal{G}^{\prime}[e]: \Gamma\left[\Gamma_{d s}\right](x)$

or $\mu_{x} \neq$ lent and

- $\mathcal{D}_{x}^{\prime}: \Gamma\left[\Gamma_{d s}\right] ; x s s^{\prime \prime}\left[x s^{\prime \prime}\right] ; y s^{\prime} \vdash \mathcal{G}^{\prime}[e]: \Gamma\left[\Gamma_{d s}\right](x)$ with $x \in x s^{\prime \prime}$ and

- $x s s^{\prime} x s^{\prime}=x s s^{\prime \prime} x s^{\prime \prime}$.

In both cases, applying the inductive hypothesis to $\mathcal{G}^{\prime}$ we derive that $\mathcal{D}^{\prime}$ : $\Gamma\left[\Gamma_{d s}\right]\left[\Gamma_{\mathcal{E}}\right] ; x s s^{\prime \prime \prime} ; y s^{\prime \prime \prime} \vdash e: T^{\prime \prime}$ for some $x s s^{\prime \prime \prime}, y s^{\prime \prime \prime}$ and $T^{\prime \prime}$ such that

(A) $\left(x s s^{\prime} x s^{\prime}=x s s^{\prime \prime} x s^{\prime \prime}\right) \backslash \operatorname{dom}\left(\Gamma_{\mathcal{G}^{\prime}}\right) \sqsubseteq x s s^{\prime \prime \prime} x s^{\prime \prime \prime}$, and

(B) the last rule applied in $\mathcal{D}^{\prime}$ is a structural rule.

Since $\Gamma_{\mathcal{G}}=\Gamma_{d s}\left[\Gamma_{\mathcal{G}^{\prime}}\right]$, from (a), (A) and transitivity of $\sqsubseteq$ we derive

$$
\left(\begin{array}{lll}
\text { xss } & x s
\end{array}\right) \backslash \operatorname{dom}\left(\Gamma_{\mathcal{G}}\right) \sqsubseteq x s s^{\prime \prime \prime} x s^{\prime \prime \prime} .
$$

This proves clause 1 .

Let $e^{\prime}$ is such that $\mathcal{D}^{\prime \prime}: \Gamma\left[\Gamma_{d s}\right]\left[\Gamma_{\mathcal{E}}\right] ; x s s^{\prime \prime} ; y s^{\prime \prime} \vdash e^{\prime}: T^{\prime \prime}$. By induction hypothesis on $\mathcal{G}^{\prime}$, substituting $\mathcal{D}^{\prime}$ with $\mathcal{D}^{\prime \prime}$ in $\mathcal{D}_{x}$ (or $\mathcal{D}_{x}^{\prime}$ ) we obtain $\Gamma\left[\Gamma_{d s}\right] ; x s s^{\prime} ; y s^{\prime} \vdash$ $\mu_{x} C_{x} x=\mathcal{G}^{\prime}[e] ; x=\mathcal{G}^{\prime}[e] ;$ OK. Applying rule (T-вцоск) we get a derivation for $\Gamma ; x s s ; y s \vdash$ $\left\{d s^{\prime} T_{x} x=\mathcal{G}^{\prime}\left[e^{\prime}\right] ; d s^{\prime \prime} v\right\}: T$, which proves clause 2 .

The proofs for the other general contexts similar and easier.

\section{Proof of Lemma 13}

1. From Lemma 12 , taking $\mathcal{G}=[] . f_{i}$, we have that

(a) $\Gamma ; x s s^{\prime}\left[x s^{\prime}\right] ; y s^{\prime} \vdash$ new $C(x s)$ : mut $C$ (ending with with an application of (T-New)) and

(b) $x s s \quad x s=x s s^{\prime} x s^{\prime}$. 
From (a) we get $\Gamma_{\mathcal{E}} ; x s s^{\prime} ; y s^{\prime} \vdash x_{i}: \mu_{i} C_{i}$.

If $\mu_{i}=$ imm, then from Lemma 5 we have that $\Gamma$; xss; ys $\vdash x_{i}: \mu_{i} C_{i}$. From rule (T-Field-Access) derive that $\mu=\mu_{i}$. Therefore, from (b) we get $\Gamma ; x s s ; y s \vdash x_{i}: \mu C_{i}$.

Let $\mu_{i}=$ mut. If $x_{i} \in x s$, from (a) we derive $x_{i} \in x s^{\prime}$. Therefore, from (b) $x s s=x s s^{\prime}$. Since $\mu \geq$ mut (with an application of (T-SuB) if needed) we derive $\Gamma_{\mathcal{E}} ; x s s ; y s \vdash x_{i}: \mu C_{i}$. If $x_{i} \in x s s$, then before rule (T-New) we have to apply (T-Swap) (to exchange the group containing $x_{i}$ with $x s$ ). Since rule (T-NEw) produces a type with mut modifier, applying rule (T-SwAP) we get a lent modifier. Therefore from (b) also $\mu=$ lent and $\Gamma$; xss; ys $\vdash x_{i}: \mu C_{i}$.

2. If $r v=$ new $C(x s)$, the result follows from clause 1 of this lemma.

Let $r v=\{d v s w\}$ where $d v s=T_{1} z_{1}=r v_{1} ; \cdots T_{n} z_{n}=r v_{n}$; , and $w=$ new $C(x s)$ or $w=x$ and $T_{i}=\mu_{i} C_{i}$. If $\Gamma$; xss $[x s] ; y s \vdash r v \cdot f_{i}: \mu^{\prime} C_{i}$, from rule (T-FIELD-ACCESS), we have that

(a) $\Gamma ; x s s^{\prime}\left[x s^{\prime}\right] ; y s^{\prime} \vdash\{d v s w\}: \mu C$, and

(b) if $\mu_{i} \neq$ mut then $\mu^{\prime}=\mu_{i}$, otherwise $\mu^{\prime}=\mu$

From Lemma 7 we have

(c) $\Gamma\left[\Gamma_{d s}\right] ; x s s^{\prime}\left[x s^{\prime}\right] ; y s^{\prime} \vdash d v s$ OK

(d) $\Gamma\left[\Gamma_{d v s}\right] ; x s s^{\prime}\left[x s^{\prime}\right] ; y s^{\prime} \vdash w: \mu^{\prime \prime} C$

(e) $(x s s x s) \backslash \operatorname{dom}\left(\Gamma_{d s}\right) \sqsubseteq x s s^{\prime} x s^{\prime}$ and

(f) $\mu \neq$ imm and $\mu \neq$ capsule implies $\mu^{\prime \prime} \leq \mu$.

If $w=$ new $C(x s)$, then $\operatorname{get}(r v, i)=\left\{d v s x_{i}\right\}$. From (b), (c) and rule (T-BLOCK)

(g) $\Gamma ; x s s^{\prime}\left[x s^{\prime}\right] ; y s^{\prime} \vdash\left\{\right.$ dvs $\left.x_{i}\right\}: \mu_{i} C_{i}$

If $\mu_{i} \neq$ mut, from (b) the result holds.

If $\underline{\mu_{i}=\mathrm{mut}}$, and $\mu \leq \mathrm{imm}$, then (a) is obtained by either rule (T-CAPsule) or (Т-Iмм). The same recovery rule can be applied to (g). If $\mu \geq$ mut, result is obtained by (g) applying rule (T-suB), if needed.

If $w=x$, then get $(r v, i)=\{d v s v\}$ if $d v s(x)={ }_{-} x=r v^{\prime}$; and $\operatorname{get}\left(r v^{\prime}, i\right)=v$. In this case the result follow by induction on $r v^{\prime}$.

3. By case analysis on the result of get $(r v, i)$, using the proof of the previous case we can see that, typing depends only on $\Gamma$ and therefore the result holds.

Proof of Lemma 14. From rule (T-Field-Assign), we have that $\Gamma$; xss $[x s]$; ys $\vdash$ $x$ : mut $C$ for some $C$ and $\Gamma$; xss $[x s]$; ys $\vdash u: \mu_{-}$where $\mu=$ mut or $\mu=$ imm. Therefore, from Lemma 5 we have that $\Gamma(x) \leq \operatorname{mut} C$, and $x \in x s$. Since $\mu=$ mut or $\mu=$ imm, from Canonical Form Theorem. 2 and 3 we have that for

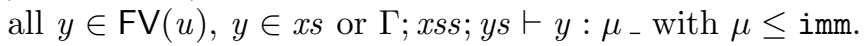

Proof of Theorem 15 (rules (FIELd-ASSIGN), and (мUT-Move))

Consider rule (FIELD-ASSIGN). Let $d s^{\prime}=d v s \mu_{x} C x=$ new $C(x s) ; T_{z} z=\mathcal{E}_{z}[x . f=u] ; d s$,

(1) $e=\mathcal{E}\left[\left\{d v s \mu_{x} C x=\right.\right.$ new $\left.\left.C(x s) ; T_{z} z=\mathcal{E}_{z}[x . f=u] ; d s v\right\}\right]$, and

(2) $e^{\prime}=\mathcal{E}\left[\left\{d v s \mu_{x} C x=\right.\right.$ new $\left.\left.C\left(x s^{\prime}\right) ; T_{z} z=\mathcal{E}_{z}[u] ; d s v\right\}\right]$, 
where fields $(C)=T_{1} f_{1} ; \ldots T_{n} f_{n}$; with $f=f_{i}$ and

(3) $\mu \geq$ mut,

(4) $x\left\|\mathrm{HB}\left(\mathcal{E}_{z}\right), u\right\| \mathrm{HB}\left(\mathcal{E}_{z}\right)$ and

(5) $x s^{\prime}$ is obtained by $x s$ replacing $x_{i}$ with $u$.

Let $\Gamma^{\prime}=\Gamma_{\mathcal{E}}\left[\Gamma_{d s^{\prime}}\right]$, from (1) and Lemma 121 for some $T^{\prime}$, xss and $y s$

(a) $\Gamma^{\prime}\left[\Gamma_{\mathcal{E}_{z}}\right] ; x s s[x s] ; y s \vdash x . f=u: T^{\prime}$ and

(b) the last rule applied in the derivation is (T-FIelD-ASSIGN).

From (1), Lemma 12,1 and Lemma 7, for some $x s s^{\prime}$ and $y s^{\prime}$

(c) $\Gamma^{\prime} ; x s s^{\prime}\left[x s^{\prime}\right] ; y s^{\prime} \vdash \mu_{x} C x=$ new $C(x s)$; OK, i.e.,

i. $\Gamma^{\prime} ; x s s^{\prime}\left[x s^{\prime}\right] ; y s^{\prime} \vdash$ new $C(x s): \Gamma^{\prime}(x)$ if $\mu_{x} \neq$ lent

ii. $\Gamma^{\prime} ; x s s_{x}\left[x s_{x}\right] ; y s^{\prime} \vdash$ new $C(x s): \Gamma^{\prime}(x)$ if $\mu_{x}=$ lent and $x s s^{\prime} x s^{\prime}=$ $x s s_{x} x s_{x}$ where $x \in x s_{x}$

(d) $\left(x s s^{\prime} x s^{\prime}\right) \backslash \operatorname{dom}\left(\Gamma_{\mathcal{E}_{z}}\right) \sqsubseteq x s s x s$.

From (a) and rule (T-Field-FILEd -Assign) we get $T^{\prime}=T_{i}$

(e) $\Gamma^{\prime}\left[\Gamma_{\mathcal{E}_{z}}\right] ; x s s[x s] ; y s \vdash u: T^{\prime}$

(f) $x \in x s$, and

(g) from Lemma 14, and (4): for all $y \in \mathrm{FV}(u)$ such that $\Gamma^{\prime}\left[\Gamma_{\mathcal{E}_{z}}\right] ; x s s ; y s \vdash y: \mu_{-}$ with $\mu \geq$ mut we have that $y \in x s$

From (c).i and (c).ii and rule (T-New), we have that $\Gamma^{\prime} ; x s s^{\prime}\left[x s^{\prime}\right] ; y s^{\prime} \vdash x_{i}: T^{\prime}$ (if $\mu_{x} \neq$ lent) or $\Gamma^{\prime} ; x s s_{x}\left[x s_{x}\right] ; y s^{\prime} \vdash x_{i}: T^{\prime}$ (otherwise). Note that, in both cases $x$ is in the current mutable group. Therefore, from (d), (g), (e), (4), and Lemma 10 we have that $\Gamma^{\prime} ; x s s^{\prime}\left[x s^{\prime}\right] ; y s^{\prime} \vdash u: T^{\prime}$ (if $\mu_{x} \neq$ lent) or $\Gamma^{\prime} ; x s s_{x}\left[x s_{x}\right] ; y s^{\prime} \vdash u$ : $T^{\prime}$ (otherwise). From rule (T-New), (5) and Lemma 122

(h) $\Gamma^{\prime} ; x s s^{\prime}\left[x s^{\prime}\right] ; y s^{\prime} \vdash \mu_{x} C x=$ new $C\left(x s^{\prime}\right)$; OK,

Let $d s^{\prime \prime}=d v s \mu_{x} C x=$ new $C\left(x s^{\prime}\right) ; T_{z} z=\mathcal{E}_{z}[x . f=u] ; d s, \mathcal{E}^{\prime}=\mathcal{E}\left[\Gamma_{d s^{\prime}}\left[\mathcal{E}_{z}\right]\right]$, and $\mathcal{E}^{\prime \prime}=\mathcal{E}\left[\Gamma_{d s^{\prime \prime}}\left[\mathcal{E}_{z}\right]\right]$. By definition of type context $\Gamma_{\mathcal{E}^{\prime \prime}}=\Gamma_{\mathcal{E}^{\prime}}=\Gamma^{\prime}\left[\Gamma_{\mathcal{E}_{z}}\right]$. Therefore, from $(\mathrm{h}),(\mathrm{a})$, and $(\mathrm{e})$

- $\Gamma_{\mathcal{E}^{\prime \prime}} ; x s s[x s] ; y s \vdash x . f=u: T^{\prime}$, and

- $\Gamma_{\mathcal{E}^{\prime \prime}} ; x s s[x s] ; y s \vdash u: T^{\prime}$.

From Lemma 122 we get $\vdash e^{\prime}: T$.

Consider rule (мuт-моve). In this case

(1) $e=\mathcal{E}\left[\left\{d v s^{\prime} \mu_{x} C x=\left\{d v s d v s^{\prime \prime} v\right\} ; d s^{\prime} v^{\prime}\right\}\right]$, and

(2) $e^{\prime}=\mathcal{E}\left[\left\{d v s^{\prime} d v s \mu_{x} C x=\left\{d v s^{\prime \prime} v\right\} ; d s^{\prime} v^{\prime}\right\}\right]$,

where

(3) $\mu_{x} \geq$ mut,

(4) $\left\{d v s^{\prime} d s^{\prime} v^{\prime}\right\} \| \operatorname{dom}(d v s)$ and 
(5) $d v s \| \operatorname{dom}\left(d v s^{\prime \prime}\right)$.

Let $d s=d v s^{\prime} \mu C x=\left\{d v s d v s^{\prime \prime} v\right\} ; d s^{\prime}$. From $\vdash e: T$ and Lemma 12 we get that, for some $T_{b}, x s s^{\prime \prime}, x s^{\prime \prime}$, and $y s^{\prime \prime}$,

$(*) \Gamma_{\mathcal{E}} ; x s s^{\prime \prime}\left[x s^{\prime \prime}\right] ; y s^{\prime \prime} \vdash\left\{d s v^{\prime}\right\}: T_{b}$

Let $\Gamma=\Gamma_{d v s^{\prime}}, \Gamma_{d s^{\prime}} x: \mu C$. From $(*)$ and Lemma 7 for some $T^{\prime}, \mu^{\prime}, x s s^{\prime}, x s^{\prime}$, and $y s^{\prime}$

(A) $\Gamma_{\mathcal{E}}[\Gamma] ; x s s^{\prime}\left[x s^{\prime}\right] ; y s^{\prime} \vdash d v s^{\prime} d s^{\prime} \mathrm{OK}$,

(B) $\Gamma_{\mathcal{E}}[\Gamma] ; x s s^{\prime}\left[x s^{\prime}\right] ; y s^{\prime} \vdash\left\{d v s d v s^{\prime \prime} v\right\}$ OK, i.e.

i. $\Gamma_{\mathcal{E}}[\Gamma] ; x s s^{\prime}\left[x s^{\prime}\right] ; y s^{\prime} \vdash\left\{d v s d v s^{\prime \prime} v\right\}: \Gamma_{\mathcal{E}}[\Gamma](x)$ if $\mu_{x} \neq$ lent

ii. $\Gamma_{\mathcal{E}}[\Gamma] ; x s s_{x}\left[x s_{x}\right] ; y s^{\prime} \vdash\left\{d v s d v s^{\prime \prime} v\right\}: \Gamma_{\mathcal{E}}[\Gamma](x)$ if $\mu_{x}=$ lent and $x s s^{\prime} x s^{\prime}=$ $x s s_{x} x s_{x}$ where $x \in x s_{x}$

(C) $\Gamma_{\mathcal{E}}[\Gamma] ; x s s^{\prime}\left[x s^{\prime}\right] ; y s^{\prime} \vdash v^{\prime}: T^{\prime}$

(D) $\left(x s s^{\prime \prime} x s^{\prime \prime}\right) \backslash \operatorname{dom}(\Gamma) \sqsubseteq x s s^{\prime} x s^{\prime}$

Let $\Gamma^{\prime}=\Gamma_{d v s d v s^{\prime}}$. From (B), and Lemma 7, for some $T^{\prime \prime}, x s s$, and $y s$ we have that

(a) $\Gamma_{\mathcal{E}}\left[\Gamma\left[\Gamma^{\prime}\right]\right] ; x s s ; y s \vdash d v s \mathrm{OK}$,

(b) $\Gamma_{\mathcal{E}}\left[\Gamma\left[\Gamma^{\prime}\right]\right] ; x s s ; y s \vdash d v s^{\prime \prime} \mathrm{OK}$, and

(c) $\Gamma_{\mathcal{E}}\left[\Gamma\left[\Gamma^{\prime}\right]\right] ; x s s ; y s \vdash v: \mu C$ with $\mu \leq \mu_{x}$

From (4) and the fact that we can assume that $x \notin \operatorname{dom}(d v s)$, we derive $\Gamma\left[\Gamma_{d v s d v s^{\prime \prime}}\right]=\Gamma, \Gamma_{d v s}\left[\Gamma_{d v s^{\prime \prime}}\right]$. Therefore, from (b), (c), and rule (Т-вцоск), followed by rule (T-suB) (in case $\mu<\mu_{x}$ ) we derive

(C1) $\Gamma_{\mathcal{E}}\left[\Gamma, \Gamma_{d v s}\right] ; x s s^{\prime} ; y s^{\prime} \vdash\left\{d v s^{\prime \prime} v\right\}: \mu_{x} C$

From (a), (5), and Lemma 10 we have

(a1) $\Gamma_{\mathcal{E}}\left[\Gamma, \Gamma_{d v s}\right] ; x s s^{\prime} ; y s^{\prime} \vdash d v s \mathrm{OK}$

From (4), the fact that, for well-formedness of declarations $x \notin \operatorname{dom}(d v s)$, and Lemma 10, we derive

(A1) $\Gamma_{\mathcal{E}}\left[\Gamma, \Gamma_{d v s}\right] ; x s s^{\prime} ; y s^{\prime} \vdash d v s^{\prime} \mathrm{OK}$,

(B1) $\Gamma_{\mathcal{E}}\left[\Gamma, \Gamma_{d v s}\right] ; x s s^{\prime} ; y s^{\prime} \vdash d s^{\prime} \mathrm{OK}$, and

(D1) $\Gamma_{\mathcal{E}}\left[\Gamma, \Gamma_{d v s}\right] ; x s s^{\prime} ; y s^{\prime} \vdash v^{\prime}: T^{\prime}$

Therefore, from (A1), (B1), (C1), (D1), (a1), (E) and (F), applying rule (T-Lock), we derive

$$
\Gamma_{\mathcal{E}} ; x s s^{\prime \prime} ; y s^{\prime \prime} \vdash\left\{d v s^{\prime} d v s \mu C x=\{d s v\} ; d s^{\prime} v^{\prime}\right\}: T_{b} .
$$


From Lemma 12 2, we derive $\vdash e^{\prime}: T$.

Proof of Lemma 16 . By induction on $e$.

If $e=x$ or $e=$ new $C(x s)$, then $e$ is a well-formed value.

If $e$ is $v \cdot f$, or $v \cdot m(v s)$, or $v \cdot f=v$, then with $\mathcal{E}=[]$ and $\rho=e$ we have that $e=\mathcal{E}[\rho]$.

If $e=$ new $C(v s)$ and $\exists v \in\{v s\} \operatorname{not} \operatorname{Var}(v)$, then applying congruence (New) and (Body) we get $e \cong\left\{T_{1} y_{1}=v_{1} ; \cdots T_{n} y_{n}=v_{n}\right.$; new $\left.C(x s)\right\}$. Therefore, either for all $i, 1 \leq i \leq n, \models_{s t} T y_{i}=v_{i}$; , or there is an $i, 1 \leq i \leq n$, such that $\forall \forall_{s t} T y_{i}=v_{i} ;$. In the first case the lemma holds, and in the second with $\mathcal{E}=[$ ] and $\rho=\left\{T_{1} y_{1}=v_{1} ; \cdots T_{n} y_{n}=v_{n}\right.$; new $\left.C(x s)\right\}$ we have that $e=\mathcal{E}[\rho]$.

Let us now consider blocks.

If $e=\{d v s v\}$, since $\{d v s v\}$ is a value, from Proposition 2, either $\{d v s v\} \cong x$ or $\{d v s v\} \cong r v$ for some $=r v$. In the first case, and if $r v=$ new $C(x s)$, then the lemma holds. In the second, let $r v=\left\{d v s^{\prime} w\right\}$; either for all $d v \in d v s^{\prime}$ we have that $\models_{s t} d v$, or there is $d v^{\prime} \in d v s^{\prime}$ such that $\nvdash_{s t} d v^{\prime}$, in which case with $\mathcal{E}=[]$ and $\rho=\left\{d v s^{\prime} w\right\}$ we have $e \cong \mathcal{E}[\rho]$.

If $e=\left\{d v s \quad T x=e^{\prime} ; d s v\right\}$ with $\models_{s t} d v s$, we have that either $e^{\prime}=v^{\prime}$ and $\forall{ }_{s t} T x=v^{\prime}$; or $e^{\prime}$ is not a value.

In the first case, define $\mathcal{E}=[$ ] and $\rho=e$, we derive that $e=\mathcal{E}[\rho]$. In the second, by induction hypothesis on $e^{\prime}$, we have that $e^{\prime}=\mathcal{E}^{\prime}[\rho]$ for some $\mathcal{E}^{\prime}$ and $\rho$. Define $\mathcal{E}=\left\{d v s T x=\mathcal{E}^{\prime} ; d s v\right\}$. We get that $e=\mathcal{E}[\rho]$. 